\title{
Analyse zweier transformativer Forschungsprojekte
}

Um herauszufinden, ob durch die von den transformativen Forschungsprojekten geführten Diskurse schon ein Wandel in Wuppertal stattgefunden hat, werden im Folgenden die beiden Fälle zunächst getrennt voneinander analysiert und später verglichen. So können Rückschlüsse darauf gezogen werden, welche Charakteristika der Projekte möglicherweise die Erfolgsaussichten erhöht oder verringert haben. Dazu wurden jeweils die transkribierten Interviews sowie die Dokumente einer Inhaltsanalyse unterzogen und mithilfe der Dokumentenanalyse jeweils die Verbreitung der in den Fällen vertretenen Ideen untersucht.

In der folgenden Darstellung (Abschn. 5.1) wird auf die aus der Theorie des diskursiven Institutionalismus herausgearbeiteten Kategorien (siehe Abschn. 3.5) eingegangen, zunächst auf die vorhandenen Akteur*innen und Ideen, dann auf den institutionellen Kontext des Projektes, auf vorhandene Herausforderungen und relevante externe Veränderungen. Danach wird auf die Anschlussfähigkeit, Umsetzbarkeit und Relevanz der Projektideen eingegangen und auf bereits wahrgenommene Veränderungen beziehungsweise Umsetzungen der Ideen. Im letzten Teil des Kapitels wird auf die Prognosen der Kongruenzanalyse (siehe Abschn. 4.3) Bezug genommen und abgeglichen, ob die vom diskursiven Institutionalismus prognostizierte Situation in der Empirie beobachtet werden kann. In Abschnitt 5.2 werden die Analyseergebnisse des zweiten Falles mit demselben Vorgehen geschildert, in Abschnitt 5.3 folgt dann ein Vergleich der beiden analysierten Fälle.

Elektronisches Zusatzmaterial Die elektronische Version dieses Kapitels enthält Zusatzmaterial, das berechtigten Benutzern zur Verfügung steht https://doi.org/10.1007/978-3-658-32601-2_5. 


\subsection{Wohlstandsindikatoren für Wuppertal}

\subsubsection{Akteur*innen und Ideen}

\subsubsection{Institutionelle Unternehmer*innen und deren Ideen}

Erste Voraussetzung für erfolgreichen Wandel ist das Vorhandensein von institutionellen Unternehmer*innen, die Ideen voranbringen. Diese benötigen außerdem Ressourcen, um ihre Ideen zu verbreiten. Auch welches Ansehen und welche Netzwerke die institutionellen Unternehmer*innen haben, spielt laut diskursivem Institutionalismus für den Erfolg eine Rolle (siehe Abschn. 3.3.2) und soll im Folgenden analysiert werden.

Im Projekt zur Entwicklung der Wuppertaler Wohlstandsindikatoren waren zwei wissenschaftliche Institute Teil des Projektkonsortiums (DI2, DI5, DI11, DI38, DI40) ${ }^{1}$ : Das Transzent der BUW und das WI. In beiden Institutionen arbeiteten Personen für das Projekt, das für drei Jahre vom BMBF gefördert wurde (I1-4, DI39). Ressourcen, um eine Idee voranzubringen, die laut Campbell (2004, S. 178 f., siehe Abschn. 3.3.2) wichtiger Faktor für erfolgreiche Veränderung sind, waren also vorhanden. Mit dem Projekt wurden unterschiedliche Ideen verfolgt. So sollte einerseits der BLI der OECD auf eine Stadt angepasst und am Beispiel Wuppertals herausgefunden werden, was Lebensqualität oder Wohlstand in dieser Stadt bedeutet beziehungsweise ob der BLI geeignet ist, diese abzubilden (DI2, DI7-9, DI14-15, DI29, DI32-34, DI37). Damit zusammenhängend sollte herausgefunden werden, wie Wohlstand und Ressourcenverbrauch sich entkoppeln lassen, wie also ressourcenleichter Wohlstand in Wuppertal aussieht (DI2-3, DI5, DI7, DI18-19, DI29, DI36, DI38-40). Diese Ideen wurden als der wissenschaftliche Beitrag des Teilprojektes beschrieben (I2-4, DI5, DI7-8, DI18, DI28-29, DI33-34, DI36-40). Dieser wissenschaftliche Beitrag alleine wäre noch nicht als innovative Idee zu verstehen, die von institutionellen Unternehmer*innen in der Stadt verbreitet wurde. Andererseits wurde aber von drei der vier projektinternen Interviewten sowie in zahlreichen Dokumenten formuliert, das Projekt ziele außerdem darauf ab, einen umfassenden Blick auf Wohlstand zu etablieren, weg von rein ökonomischen Aspekten von Wohlstand und deren Messung durch das BIP hin zu weiteren Aspekten wie Gesundheit, Bildung oder Umwelt (DI5-6,

\footnotetext{
${ }^{1}$ Um die Verweise auf Interviews und Dokumente exakt, aber in kurzer Form anzugeben, wurden diese jeweils nummeriert (siehe elektronisches Zusatzmaterial). DI1-DI40 stehen für die analysierten Dokumente des ersten Falles, der Indikatorenentwicklung, I1-I13 für die Expert*inneninterviews dieses Falles. DG1-DG216 bezeichnen die Dokumente über „Glücklich in Wuppertal“ und G1-G20 die dazu geführten Interviews.
} 
DI8, DI12, DI16, DI18, DI29, DI31). Ein Interviewter des Projektteams fasste dies folgendermaßen zusammen:

\begin{abstract}
„Und auch eine Kommune ist eigentlich im Wettbewerb mit anderen Kommunen immer dabei zu schauen, dass es als Wirtschaftsstandort, oder ... sich da wirklich behaupten kann. Andere Indikatoren eben wie Umwelt, Gesundheit, Bildung und so weiter rücken immer in den Hintergrund vor diesem wirtschaftlichen Wachstumsgedanken. Und gerade wenn wir über Wohlstand sprechen - im Sinne von Wohlfahrt auch, dass man sich wohl fühlt - dann ist es eben nicht nur abhängig vom BIP sondern eben auch von den anderen bereits genannten Faktoren und von vielen mehr. Und das systematisch in Beziehung zu bringen, das war einfach das Anliegen von AP $1^{2}[\ldots]$ Und damit eben auch einen weiteren Blick auf Wohlstand nach vorne bringen, der nicht eben reduziert ist auf unternehmerische Perspektive und BIP.“(I1)
\end{abstract}

Das Vorhandensein dieser Idee eines erweiterten Verständnisses von Wohlstand wurde als der transformative Beitrag des Projektes beschrieben (I1-2, I4). Die Anpassung des BLI auf Wuppertal als Better Life Index urban (BLI-u) sollte nicht nur getestet, sondern dieser daraufhin auch genutzt und als Kompass für städtische Entscheidungen dienen (I2-3, DI4, DI7, DI12, DI16, DI30-31, DI34-35, DI40). Der BLI-u sollte als alternatives Wohlstandsverständnis neues Paradigma werden, das Politik leitet (I1-2, DI8, DI31). Die Forschenden sahen es dabei auch als ihre Rolle, die Implementierung der Indikatoren in städtische Prozesse aktiv mit voranzutreiben (DI14, DI32). Hier wird also deutlich, dass die institutionellen Unternehmer*innen eine Veränderungsintension auf der Ebene eines Paradigmas, also eines Wandels dritter Ordnung, hatten.

Konkreter heruntergebrochen war außerdem die Idee vorhanden, die Indikatoren und Dimensionen könnten als Instrument und Angebot eingesetzt und dazu genutzt werden, Projekte zu bewerten (I2, DI12). Bezüglich dieser Nutzung der Dimensionen als Bewertungskriterium für kleinere Projekte argumentiert einer der Interviewten, dass diese kleineren Anwendungen als Projektbewertung zwar auch im Sinne des Projektes seien, die Wohlstandsdimensionen aber eigentlich nur als übergreifendes Leitbild bzw. Paradigma ihrem Sinn gerecht werden würden (I2). Trotzdem wird hier eine Veränderungsintension auf der Ebene von konkreten Policies deutlich, teilweise auch die Idee, dies längerfristig in Programme zu integrieren. Diese Ideen beziehen sich also auf einen von den Projektbeteiligten angestrebten Wandel erster und zweiter Ordnung.

\footnotetext{
${ }^{2}$ Das Arbeitspaket 1 umfasste im WTW-Projekt die in diesem Fall analysierte Entwicklung von Wohlstandsdimensionen und -indikatoren für Wuppertal mithilfe eines partizipativen Forschungsprozesses.
} 
Bei einem Blick auf die öffentlichen Dokumente des Projektes (Broschüren, Webseiten, Protokolle) fällt auf, dass die Beschreibung der Idee sich meist auf die Wohlstandsdimensionen bezieht, die genauen Indikatoren und deren Berechnung jedoch, wenn überhaupt, nur beispielhaft auftauchen. Die Kommunikation blieb also meist auf der Ebene der Wohlstandsdimensionen. Drei von vier projektinternen Interviewten sahen diese transformative Wirkung auf Ideen und damit auf politische Entscheidungen in der Stadt als ein Ziel (I1-2, I4). Der vierte Projektbeteiligte (I3) sagte, dies sei nicht Ziel gewesen, erwähnte es im späteren Verlauf des Interviews jedoch auch als Nebeneffekt. Die Priorisierung zwischen den Zielen wurde also unterschiedlich verstanden von verschiedenen Projektbeteiligten, die alle jedoch auch den transformativen Beitrag erkannten.



Abb. 5.1 Zuordnung der Ideen zu Veränderungsintensionen (Wohlstandsindikatoren). Die Abbildung zeigt die Zuordnung der Ideen der institutionellen Unternehmer*innen im Fall der Wohlstandsindikatoren zu den drei Graden der Veränderung nach Hall (1993). Die Wohlstandsindikatoren zur Evaluierung von Stadtentwicklungsprojekten zu nutzen würde je nach Ausgestaltung einen Wandel erster oder zweiter Ordnung bedeuten, während die Einführung eines alternativen Wohlstandskompasses eine Transformation mit sich bringen würde.

Quelle: Hall (1993) und eigene. Eigene Darstellung

Das Projekt zielte also neben dem klassischen wissenschaftlichen Beitrag auch auf eine Veränderung von Ideen unterschiedlicher Ebenen in der Stadt ab (siehe Abb. 5.1). Die Projektpartner*innen agierten als institutionelle Unternehmer*innen, indem sie ihre Idee eines erweiterten Wohlstandsverständnisses als neuen Kompass für Stadtentwicklung voranbrachten, der im Sinne eines Paradigmas (Campbell 2002, S. 22 f., 2004, S. 94 f.; Schmidt 2017, S. 251) verstanden 
werden kann und einen Wandel dritter Ordnung bezweckte. Die Wohlstandsindikatoren zur Projektbewertung zu nutzen, kann je nach konkreter Ausgestaltung dagegen als Policy oder Programm (Schmidt 2017, S. 251) verstanden werden und lediglich Wandel der ersten oder zweiten Ordnung herbeiführen.

\subsubsection{Unterstützung durch zivilgesellschaftliche Akteur*innen und Unternehmen}

Um erfolgreich Wandel herbeizuführen, unabhängig davon, auf welcher Ebene, benötigen institutionelle Unternehmer*innen Unterstützung und Netzwerke zu anderen Akteur*innen. Im Folgenden wird daher zunächst dargelegt, ob und von wem die institutionellen Unternehmer*innen des Projektes Unterstützung aus der Zivilgesellschaft oder von Unternehmen bekamen, bevor sich später anderen Gruppen von Akteur*innen zugewendet wird.

In diesem Fall wurden punktuell Vereine und engagierte Zivilgesellschaft eingebunden, insbesondere im Rahmen von Workshops zur Indikatorenentwicklung und -nutzung und in Wirkungsabschätzungsworkshops mit den im WTW-Projekt aufgebauten Reallaboren (I1-2, I4, I6, I8, I11, DI14, DI15, DI32). So fand zu Beginn des Projektes ein Workshop zur Entwicklung der Wohlstandsindikatoren statt, bei dem 20 Personen von 16 Vereinen und Initiativen teilnahmen und über Dimensionen des guten Lebens aus ihrer Sicht sprachen, diese sammelten und priorisierten (DI5). Im Nachgang fand eine Befragung statt, bei der zwar kein persönlicher Kontakt entstand, aber eine Information über das Forschungsprojekt an 1000 zufällig ausgewählte Wuppertaler*innen mit Bitte um Beantwortung eines Online-Fragebogens versendet wurde. Dieser fragte ebenfalls Aspekte des guten Lebens und deren Gewichtung sowie sozio-demografische Merkmale der Befragten ab (DI7, DI29, DI31, DI40).

In den Interviews wurden zwei Vertreter der Zivilgesellschaft besonders herausgestellt, die in die Indikatorenentwicklung involviert waren: Ein Repräsentant von Utopiastadt und ein Mitglied des Kompetenznetz Bürgerhaushalt. Beide haben die Idee der Wohlstandsindikatoren in andere städtische Kontexte getragen, beispielsweise in den Stadtentwicklungsprozess W2025 (I2) und das Bürgerbudget (I11). Daher können sie beide als Vermittler*innen (Campbell 2004, S. 104 f.) verstanden werden, die zur Verbreitung der Ideen beigetragen haben. Der Vertreter des Kompetenznetz Bürgerhaushalt könnte zusätzlich selbst auch als institutioneller Unternehmer (Campbell 2004, S. 177 f.) verstanden werden, der seine Ideen in der Stadt umsetzen will und dafür Gelegenheiten und Verbündete sucht, wo sich die Wohlstandsindikatoren anboten. Aus diesem Grund schlug er sie zur Nutzung in anderen Kontexten vor und erleichterte dadurch die Vernetzung und Diffusion, aber auch erste Umsetzungen (I9). Mit diesen beiden Vertretern konnte das Projekt 
Unterstützer gewinnen, die die Projektziele und die Idee alternativer Wohlstandskonzepte voranbrachten. Weitere zivilgesellschaftliche Akteur*innen waren eher punktuell eingebunden und gaben an, nur wenig über den Projektverlauf gewusst und nur sporadisch von Veranstaltungen erfahren zu haben, die sie später kaum mehr in Erinnerung hatten (I11-12).

Daneben wurde Kontakt zur Wirtschaftsförderung hergestellt, die als Anstalt des öffentlichen Rechts sehr enge Kontakte zur Stadtverwaltung pflegt und teilweise in städtischen Gremien vertreten ist. So hat eine Person in der Wirtschaftsförderung sich für die Ideen der Wohlstandsindikatoren eingesetzt, indem sie diese in den Kontext der Stadtentwicklungsstrategie W2025 integriert hat und so in neue Netzwerke hereingeholt hat (I2, I8). Daher kann auch sie als Vermittlerin im Sinne von Campbell (2004, S. 104 f.) verstanden werden. Der Austausch zwischen ihr und einem der Projektmitarbeitenden wurde als regelmäßig beschrieben, so dass sie über die Entwicklungen im Projekt informiert war (I8). Mit ihr konnte das Projekt also eine weitere Unterstützerin gewinnen, in diesem Fall aus einem städtischen Betrieb. Sie stellte den Kontakt zu einem städtischen Kontext her, in dem die Ideen des Projektes vorgestellt wurden und durch die die meisten der genannten Personen der Stadtverwaltung von den Ideen erfuhren.

Insgesamt konnte das Projekt also bereits einzelne Unterstützer*innen aus der Zivilgesellschaft und Unternehmen gewinnen, die sich aktiv dafür einsetzten und die Ideen voranbrachten, und erreichte während der Befragung oder dem Bürgerbudget eine größere Gruppe an Personen mit allgemeinen Informationen zu Ideen über Wohlstand. Daneben konnten bereits zwei Unterstützer*innen aus der Zivilgesellschaft sowie eine aus einem städtischen Unternehmen gewonnen werden, die die Ideen in andere Kontexte trugen.

\subsubsection{Zugang zu Entscheidungsträger*innen}

Neben Unterstützung aus der Bürgerschaft benötigen institutionelle Unternehmer*innen außerdem Zugang durch und Unterstützung von Entscheidungsträger*innen, um die Ideen in der Stadt umzusetzen (siehe Abschn. 3.3.2), welche in diesem Fall eher punktuell eingebunden wurden (DI7, DI30, DI40). Hier wurde die Statistikstelle, das Dezernat für Stadtentwicklung, der Oberbürgermeister und die Stabsstelle Bürgerbeteiligung genannt (I1-6, I8, I13). Mit dem Mitarbeiter der Statistikstelle bestand Kontakt, als es um städtische Daten über Wuppertal ging und um eine Stichprobe von Wuppertaler Einwohner*innen (I5).

Durch den Einsatz der Vertreterin der Wirtschaftsförderung wurden die Wohlstandsdimensionen bei einem Termin des Lenkungskreises der Stadtentwicklungsstrategie W2025 vorgestellt, bei dem 19 Personen, größtenteils aus der Stadtverwaltung, anwesend waren. Die Mitglieder dieses Gremiums entschieden 
sich für eine Nutzung der Dimensionen in diesem Kontext, was bisher jedoch noch nicht realisiert wurde. Mitglieder des Lenkungskreises waren Projektverantwortliche der 13 Schlüsselprojekte, die aus verschiedenen Bereichen der Verwaltung kommen: der Oberbürgermeister und sein Büroleiter, die Dezernenten für Soziales, Kultur und Sport, Beteiligung, der Zoodirektor, die Leitungen der Ressorts Stadtentwicklung und Umweltschutz, des Presseamtes und des Rechtsamtes, der Gleichstellungsstelle und des Bereichs Schulen sowie stadtnahe Betriebe und Zivilgesellschaft (DI13). Durch eine Präsentation in diesem breit besetzten Gremium konnten also Entscheidungsträger*innen über das Projekt und die damit verbundenen Ideen informiert werden.

Ein zweiter städtischer Kontext, in dem die Ideen vorgestellt wurden, waren Gespräche der Bürgerbegleitgruppe zum Bürgerbudget 2017, bei dem die Mitarbeitenden der Stabsstelle Bürgerbeteiligung sowie sieben Bürger*innen anwesend waren. Dort wurden die Dimensionen ebenfalls vorgestellt und sich für eine Nutzung dieser entschieden (I6, I9, DI17), die dann später in einer Bürgerwerkstatt mit circa 120 Teilnehmenden umgesetzt wurde. Dort wurden die Dimensionen dann erneut vorgestellt (DI20). Die Beteiligten der Bürgerbegleitgruppe haben sich also für die Dimensionen entschieden und die Projektziele und die verfolgten Ideen damit ein Stück vorangebracht.

Insbesondere bei diesen zwei Projekten gab es einen längerfristigen Austausch zwischen den Beteiligten des Projektes und der Stadtverwaltung und beide Seiten profitierten von der Zusammenarbeit. Im Fall des Bürgerbudgets war die Stabsstelle Bürgerbeteiligung auf der Suche nach Kriterien für einen Gemeinwohlcheck und nahm die Beratung der Wissenschaftler*innen gerne an, die dadurch wiederum Öffentlichkeit erzeugen und die Verwendung der Dimensionen erproben konnten (I6).

Auch zum Oberbürgermeister und dessen Büro bestand neben dem W2025Prozess zusätzlich Kontakt durch einen regelmäßigen Erfahrungsaustausch zwischen dem Oberbürgermeister und dem Leiter des WI, gleichzeitig Projektleiter (I13). Insgesamt zeigt sich also, dass diese von Campbell (2004, S. 178 f.) genannte Voraussetzung für institutionellen Wandel, Zugang zu Entscheidungsträger*innen zu haben, vorliegt. Dies kam insbesondere durch zwei Unterstützer*innen beziehungsweise Vermittler*innen zustande, die die Ideen in städtische Kontexte trugen. Dieser Austausch wurde aber nur in Einzelfällen intensiviert und es ist bisher keine größere Unterstützung von Entscheidungsträger*innen zu beobachten. Darauf, welche der Ideen die Entscheidungsträger*innen wahrnahmen und gegebenenfalls unterstützten, wird später genauer eingegangen. 


\subsubsection{4 Öffentliche Diskurse und Diffusion der Ideen}

Neben dem Zugang zu Entscheidungsträger*innen und der Unterstützung verschiedener Akteur*innen könnte außerdem eine öffentliche Diskussion über die angesprochenen Ideen einen Wandel wahrscheinlicher machen beziehungsweise eine Grundlage dafür schaffen. Darüber hinaus kann an der Verbreitung der Ideen über die Wohlstandsindikatoren im städtischen Diskurs erkannt werden, wie weit die Ideen diffundiert sind und ob es bereits zu einem diskursiven Wandel gekommen ist. Nutzen bereits andere Akteur*innen die Ideen und Begriffe des Projektes, wer agiert in dem Diskurs und wer wurde dadurch erreicht? Um herauszufinden, wie weit die öffentliche Kommunikation der Ideen ging und ob es schon zu einer diskursiven Veränderung kam, wurden diese öffentlichen Diskurse und Kommunikationskanäle ebenfalls untersucht.

Die Prozesse und Ideen des Projektes wurden hauptsächlich über die eigenen Kanäle wie Blog, Webseiten oder Broschüren verbreitet (I1-2, I4). Artikel in Zeitungen und anderen Pressemedien sind nur wenige erschienen, was auch die Ergebnisse der Dokumentenanalyse bestätigen. So wird in 24 der 40 gefundenen Dokumente die Indikatorenentwicklung nur am Rande erwähnt und es wird hauptsächlich etwas anderes oder ein allgemeineres Thema behandelt. Bei den 16 Dokumenten, die hauptsächlich das Projekt der Indikatorenentwicklung thematisieren, handelt es sich zur Hälfte um nichtöffentliche projektinterne Dokumente wie Protokolle oder Transkripte von Workshops. Die anderen acht Dokumente, bei denen die Indikatorenentwicklung im Mittelpunkt steht, sind öffentliche Dokumente.

Von allen öffentlich verfügbaren Dokumenten, die in die Analyse einbezogen wurden, sind neun Einträge auf eigenen Webseiten der Projektpartner*innen und vier auf einem eigenen Blog veröffentlicht. Bei fünf Dokumenten handelt es sich um Projektberichte wie Broschüren oder Kurzinformationen und bei zwei um Artikel in wissenschaftlichen Zeitschriften. Auf diese wurde jeweils ebenfalls auf der Projekthomepage verwiesen. Ein wissenschaftliches Poster und fünf wissenschaftliche Vorträge wurden auf Tagungen präsentiert und die dazugehörigen Abstracts oder Präsentationsfolien auf der projekteigenen Webseite veröffentlicht. Daneben berichtete die Presse in vier Artikeln in den Lokalzeitungen unter anderem über die Ideen der Indikatorenentwicklung sowie in einem Radiobeitrag. Zusätzlich sind auf Internetseiten anderer Anbieter dreimal Artikel auf Blogs und in Newsbereichen anderer Akteur*innen zu finden.

Bei der Dokumentenanalyse wird deutlich, dass in der zweiten Hälfte des Untersuchungszeitraumes mehr Dokumente über die Indikatorenentwicklung veröffentlicht wurden. Während 2015 fünf Dokumente entstanden, 2016 elf, entstanden im ersten Halbjahr 2017 bereits elf Dokumente, im zweiten Halbjahr weitere 
vier. 2018 wurden bis zum Ende des Untersuchungszeitraumes und damit auch Ende der Förderlaufzeit des Projektes zehn Dokumente verfasst, von denen die meisten auch öffentlich verfügbar sind. Einige der 2016 beispielsweise im Zusammenhang mit wissenschaftlichen Präsentationen entstandenen Dokumente wurden erst im Laufe des Jahres 2018 hochgeladen, um zum Projektende hin die Ergebnisse Interessierten zur Verfügung zu stellen. Autor*innen der meisten Dokumente waren die Projektmitarbeitenden, also die institutionellen Unternehmer*innen selbst oder sehr eng daran Beteiligte (wie die Pressestellen der beteiligten Organisationen). Acht Dokumente wurden von Journalist*innen oder anderen Personen verfasst, die nicht zu den projektdurchführenden Einrichtungen gehörten.

Schlussfolgernd aus den Kanälen, über die die Dokumente verbreitet wurden, sowie deren Inhalte, hat sich herausgestellt, dass Zielgruppe von circa der Hälfe der Dokumente (19) unter anderem die Bürgerschaft in Wuppertal und der Region ist, beispielsweise bei Broschüren und Presseartikeln. Ebenfalls 19 Dokumente richten sich unter anderem an Akteur*innen der Wissenschaft und Wissenschaftspolitik oder Fördergeber, wobei es hier einige Überschneidung mit der Zielgruppe Bürgerschaft gibt. Die nichtöffentlichen Dokumente sind an Projektmitarbeitende oder Teilnehmende von Sitzungen gerichtet und dienen der Dokumentation des Prozesses.

Die meisten Dokumente berichten direkt über Veranstaltungen im Rahmen des Projektes oder über Projektergebnisse. Neunmal werden die Indikatoren auch im Zusammenhang mit Bürgerbeteiligung in Wuppertal genannt, alle im Zusammenhang mit der Nutzung der Wohlstandsdimensionen in einer Bürgerwerkstatt des Bürgerbudget-Prozesses, bei dem parallel mehrere Protokolle an mehreren Tischgruppen angefertigt wurden. Zusätzlich werden die Indikatoren in zwei Dokumenten im Rahmen einer Sitzung des Lenkungskreises der Stadtentwicklungsstrategie W2025 genannt. Achtmal wurde das Projekt in wissenschaftlichen Abhandlungen erwähnt und analysiert.

Die Wohlstandsindikatoren tauchen in keinem öffentlichen städtischen Dokument auf, sondern lediglich in einem Protokoll einer städtischen Sitzung zur Stadtentwicklungsstrategie W2025. Die Ideen scheinen also nicht Schwerpunkt in städtischen Prozessen geworden zu sein. Die größte Zahl der Dokumente wurde von den institutionellen Unternehmer*innen des Projektes selbst oder über ihre Kanäle veröffentlicht, wodurch davon ausgegangen werden kann, dass nur ein spezifischer Teil der Bevölkerung in Wuppertal mit den Ideen erreicht wurde, die schon zuvor an den Themen interessiert waren. Dies wurde auch in den Interviews mit den Projektbeteiligten so eingeschätzt (I1-4). Dort und in einigen Dokumenten wurde außerdem angemerkt, dass hauptsächlich engagierte Personen mit hohem Bildungsstatus und meist ohne Migrationshintergrund von den Ideen 
erfuhren und diese nicht in die Breite der Bevölkerung getragen wurden (I1-2, I4, DI5, DI8, DI21-26, DI29-31, DI40). Auch die Interviewten außerhalb des Projektes merkten an, dass bisher noch wenig Öffentlichkeitsarbeit geschehen ist, was jedoch in der verfügbaren Zeit kaum möglich gewesen wäre (I7-8) und darüber hinaus verschiedene Bevölkerungsgruppen unterschiedlich angesprochen werden müssten (I11).

Dies lässt schlussfolgern, dass die Ideen des Projektes vor allem die bereits involvierten oder über die Arbeit der Institute informierten Akteur*innen erreicht haben und insbesondere die eigenen Kanäle verwendet wurden. Darüber hinaus sind die Ideen des Projektes kaum in die Breite der Bevölkerung diffundiert. Neben der lokalen Bürgerschaft wurden andere Wissenschaftler*innen sowie Wissenschaftsförderer adressiert. Die Entscheidungsträger*innen wurden dagegen in keinem Dokument explizit angesprochen und andersherum spielen die Ideen auch bisher in keinem öffentlichen städtischen Dokument eine Rolle. Möglicherweise lässt sich aus der geringen Resonanz in den lokalen Medien auf mangelnde Anschlussfähigkeit oder wenig Vernetzung und Öffentlichkeitsarbeit schließen, worauf in Abschnitt 5.1.3 genauer eingegangen wird. Ob diese Kommunikation in ausgewählte Kreise der Zivilgesellschaft hinein statt in die Breite die Wahrscheinlichkeit für erfolgreiche Veränderungen verringerte, wird ebenfalls noch an späterer Stelle untersucht.

\subsubsection{Kommunikation der Ideen}

Nachdem nun allgemein die Verbreitung der Ideen dargestellt wurde, wird nun dargelegt, von welchen Ideen die Personen aus Zivilgesellschaft und Stadtverwaltung beziehungsweise städtischen Betrieben Kenntnis bekommen haben und ob diese Wahrnehmung sich von den ursprünglichen Ideen der institutionellen Unternehmer*innen unterscheidet, ob also die Übersetzung zu einer Abwandlung der Ideen geführt hat. Daran kann erkannt werden, inwieweit welcher Teil der Ideen diffundiert ist und wie gut die Kommunikation dieser Ideen in verschiedene Netzwerke hinein gelungen ist.

Von den externen Interviewten wurde einerseits der wissenschaftliche Beitrag genannt, herauszufinden, was Wohlstand oder gutes Leben in Wuppertal über rein materielle Aspekte hinaus ausmacht (I11-12), dafür ein Indikatorenset zu entwickeln und zu messen (I8-9, I13). Im Laufe des Gesprächs erinnerte sich ein Interviewter der Zivilgesellschaft wieder an die Bedeutung des Begriffes Wohlstand:

„Also für mich ist wirklich Wohlstand insgesamt eine weitere Zufriedenheit mit meiner Lebenssituation, die auch ... gut es muss eine gewisse materielle Basis sein aber 
irgendwann hört es auf, an der Stelle weiter zu gehen. Es hat sehr viel mit Sozialkontakten zu tun, sehr viel mit sozialer Zufriedenheit. Ja, hat was mit Sicherheit zu tun, mit Wohlfühlen, natürlich auch was mit Gesundheit. Und soweit ich weiß, so langsam erinnere ich mich, haben wir eigentlich ähnliche Sachen diskutiert.“ (I12)

Gleichzeitig wurde gesehen, dass hinter der Wohlstandsdefinition und dem Projekt die Idee steht, den Blick zu weiten und nicht-monetäre Aspekte von Wohlstand in den städtischen Diskurs zu bringen (I5-6, I9, I12), also eine Veränderungsintension auf der Ebene von Wandel dritter Ordnung vorhanden ist. So formulierte ein Interviewter der Stadtverwaltung die Zielsetzung als

„[...] eigentlich so diese alternative Zielsetzung, vielleicht jetzt auch diese alte Diskussion: Bruttoinlandsprodukt oder so, ist das jetzt, was ja häufig als Wohlstandsindikator genommen wird, ja letztendlich in Ermangelung anderer, ist ja dann auch schon ewig umstritten. Und dass das jetzt einfach mal so eine Alternative bietet." (I5)

Mehrere externe Interviewte verstanden die Wohlstandsindikatoren als Angebot für einen Kompass oder Instrument für städtische Entscheidungen (I5-6, I9). Von einem Interviewten wurde außerdem als Ziel gesehen, eine Transformation anzuregen und die Stadtgesellschaft zu aktivieren (I11). Diese Interviewantworten zeigen, dass von einigen der externen Personen also der gewünschte transformative Beitrag des Projektes erkannt wurde, der von den institutionellen Unternehmer*innen angestrebt wurde.

Aus den Diskussionen rund um das Stadtentwicklungskonzept W2025 wurden die Wohlstandsindikatoren außerdem als Maßstab für Lebensqualität und als Instrument zur Evaluierung von Projekten der Stadt verstanden (I7-8, DI13). Wie genau diese Evaluierung durchgeführt werden soll, wurde jedoch weder von den Interviewten noch im Protokoll eines W2025-Treffens beschrieben (I7-8, DI13). Da es sich dabei um konkrete Vorschläge für Instrumente handelt, sind hier Ideen angesprochen, die als neue Policies einen Wandel erster Ordnung bewirken können.

Einige der externen Interviewten konnten nicht alle Projektziele wiedergeben, kannten nur einen Teilbereich davon oder vermischten es gedanklich mit anderen Projekten wie der App „Glücklich in Wuppertal“ oder den Reallaboren des WTWProjektes (I5, I8-9, I11-12). Bei einer Befragten stand die subjektive Bewertung städtischer Entwicklungen im Vordergrund (I8). Auch von einigen Entscheidungsträger*innen wurde eingeräumt, dass sie über die Ergebnisse des Projektes nicht Bescheid wussten und nicht über den Verlauf informiert wurden (I5, I7). Teilweise hätten sie sich mehr Informationen gewünscht. 
„Ja, also ich fände das super, wenn es quasi so einen jährlichen, zweijährlichen Bericht, wo man halt auch dann wiederum Entwicklungen festmachen kann. Also, das fände ich für unsere Arbeit interessant. [...] Und wenn man da einfach ein institutionalisiertes Instrument hat, was von mir aus alle zwei Jahre da ist, und einfach auch dann mal über zehn Jahre vielleicht schaut: Okay, was haben... wie entwickelt sich Stadt? In der Richtung fände ich das höchst willkommen.“(I6)

Auch zwei Interviewte aus der Zivilgesellschaft hätten sich regelmäßige Informationen über das Projekt gewünscht (I9, I11). Da es sich bei diesen Personen bereits um die handelt, die oder deren zivilgesellschaftliche Initiative mindestens zweimal in Dokumenten oder Interviews genannt wurde (siehe Abschn. 4.4.2), ist davon auszugehen, dass andere Personen der Zivilgesellschaft eher noch weniger Bescheid wussten. In einem Zeitungsartikel über die Bürgerwerkstatt wurde geschrieben, dass eine Mitarbeiterin der Universität die Spielregeln der Bürgerbeteiligungsveranstaltung vorstellte, obwohl die zwölf Wohlstandsdimensionen als Kriterien für den Gemeinwohlcheck vorgestellt wurden und das Vorgehen der Veranstaltung an sich von der Moderatorin erläutert wurde (DI20). Die Inhalte und Ziele der Dimensionen wurden also nicht korrekt wiedergegeben.

Bei der Analyse der Dokumente ist außerdem auffällig, dass keines der Dokumente genauer über die eigentliche Berechnung der Indikatoren oder deren Entwicklung im Zeitverlauf berichtet, also quantitative Ergebnisse liefert. Meist bleiben die Informationen auf der Ebene der Dimensionen und nennen keine konkreten Zahlen. Zum Ende des Untersuchungszeitraumes waren auf der Webseite zu den Indikatoren nur zu drei der zwölf Wohlstandsdimensionen Zahlenreihen der Indikatoren hinterlegt. Und auch diese wurden erst spät im Laufe des Prozesses veröffentlicht, nachdem sich aus dem Beteiligungsprozess zwölf Dimensionen herausgestellt hatten. Dies wurde ebenfalls kritisch von einem Projektbeteiligten reflektiert:

„Und um glaube ich in so einen Impact zu kommen, wäre es glaube ich besser gewesen, wir hätten uns intern in einem sehr kleinen Workshop darauf verständigt: Das sind jetzt die Elf für Wuppertal. Die hauen wir jetzt mal raus. Jetzt gucken wir, wer darauf reagiert, und dann in dem Prozess passen wir sie nochmal an. Also, das war akademisch vermutlich total redlich, aber jetzt unter so einem transformativen Aspekt, den man sich gewünscht hätte, vielleicht nicht ganz optimal.“ (I4)

Es wurden also durchaus alle in diesem Zusammenhang stehenden Ideen der institutionellen Unternehmer*innen kommuniziert, es gelangte aber jeweils nur ein Teil davon zu den jeweiligen Adressaten. Die meisten externen Projektbeteiligten haben die Ziele des Projektes nicht in Gänze und nicht auf allen Ebenen von 
dahinterliegenden Ideen überblickt. Andere vermischten versehentlich die Ideen des Projektes mit anderen Projekten. Dies und auch die Darstellung in den Medien weist darauf hin, dass das Projekt schwer zu vermitteln und zu verstehen war. Dies deutet darauf hin, dass die Ideen des Projektes nicht, wie von Campbell als Kriterium für erfolgreichen institutionellen Wandel formuliert, einfach und verständlich kommuniziert wurden (Campbell 2004, S. 177 f.). Ein Interviewter eines Reallabores formulierte diese Problematik:

„Ehm, ja, ich hatte so durchweg im Projekt teilweise so das Gefühl, dass das sehr abstrakt ist irgendwie. Das fand ich jetzt irgendwie immer, wenn man von... da musste man immer aufpassen und glaube ich einen guten Weg finden, das überhaupt zu vermitteln und... ich meine gut, das war klar, dass es auch um Wissenschaft geht, deswegen ist es halt auch so. Aber das fand ich immer sehr schwierig, immer mal wieder. Selbst für mich war es teilweise nicht... [lacht] Oder mir wurden am Anfang auch ein paar Sachen nicht gesagt, oder..."(I10)

Daneben wurde teilweise der Begriff Wohlstand verwendet, teilweise von Dimensionen des guten Lebens gesprochen. Beim Bürgerbudget 2017 wurden die 12 Dimensionen außerdem noch mit dem Thema Gemeinwohl zusammengebracht. So war die Kommunikation oft nicht einheitlich. Zwar erleichterte dies, an Diskurse anzuknüpfen, führte insgesamt aber auch zu einer Unklarheit. Laut Schmidt (2006, S. 253) kann Mehrdeutigkeit auch förderlich für den Erfolg einer neuen Idee sein, da sich dann Akteur*innen unterschiedlicher Perspektiven zuordnen, was in dem Fall jedoch nur bedingt erfolgreich war. Die Vielschichtigkeit und die Komplexität wurden hier eher als Nachteil denn als Vorteil empfunden, da so die Öffentlichkeitsarbeit und Suche nach Unterstützer*innen erschwert war und die meisten das Gefühl hatten, die Ideen nicht einordnen zu können (I2, I4).

Zusammenfassend lässt sich also feststellen, dass zwar alle von den institutionellen Unternehmer*innen propagierten Ideen von einigen externen Interviewten wahrgenommen wurden, jedoch von den meisten jeweils nur ein Ausschnitt verstanden und in Dokumenten nur einzelne Aspekte erwähnt wurden. Während einigen in der Zivilgesellschaft das alternative Paradigma und damit die Intension einer Transformation klar wurde, so erfuhren die meisten Entscheidungsträger*innen lediglich von den Policies und teilweise Ideen neuer Programme, die Veränderungen erster oder zweiter Ordnung bewirken würden. Die Komplexität und Mehrdeutigkeit durch die Ideen unterschiedlicher Ebenen und unterschiedlichen Formulierungen stellten eher eine Schwierigkeit dar. Einfache und verständliche Kommunikation war hauptsächlich bezüglich der Ideen auf den Ebenen von Policies und Programmen möglich, schwieriger in Bezug auf die Paradigmen. 


\subsubsection{Rolle der Wissenschaft}

Da für die Analyse der Ideen, Akteur*innen und Veränderungsprozesse auch von Relevanz ist, wie die Wissenschaftler*innen wahrgenommen wurden und welche Funktionen sie außer der von institutionellen Unternehmer*innen noch einnahmen, widmet sich das folgende Teilkapitel nochmals dieser Gruppe mit einem Fokus auf den ausgeführten Rollen und der Wahrnehmung bei Personen außerhalb der Wissenschaft.

Das Transzent und das WI wurden von den externen Interviewten als glaubwürdig, engagiert und mit hoher Reputation beschrieben (I6, I8-9, I11, I13). Dadurch wurde es als positiv wahrgenommen, dass lediglich wissenschaftliche Partner*innen und keine Unternehmen in das Projekt involviert waren, um die Glaubwürdigkeit und Wissenschaftlichkeit zu erhalten (I6, I13). Die Wissenschaft profitierte dabei von einem Vertrauensvorschuss in der Stadt und verfügte über eine gute Stellung (I2, I4).

In den Interviews und in Dokumenten nannten die Wissenschaftler*innen selbst zwei verschiedene Rollen die sie eingenommen hätten: die klassische forschende und die transformierende Rolle, in der die Wissenschaftler*innen Stellung zu städtischen Themen beziehen (I1-4, DI6-7, DI9, DI14, DI31-32, DI37-38, DI40). Die Wissenschaftler*innen beschrieben sich selbst als transformativ Forschende, die „sozial robustes Wissen“ (Nowotny et al. 2001, S. 167) produzieren und selbst aktiv Transformation und die Implementation der Indikatoren voranbringen wollen (DI10-11) und schrieben sich damit klar die Rolle institutioneller Unternehmer*innen zu, wie bereits in Abschnitt 5.1.1.1 dargestellt. Indem die Forschenden ihre Rolle hinterfragten und an die wahrgenommenen Herausforderungen anpassten und mit der Gründung des Transzent dazu sogar eine neue Organisation geschaffen wurde, kann hier ein Ansatz einer Modus-3Wissenschaft erkannt werden (siehe Abschn. 2.2.2) Auch wenn die Wissenschaft immer wieder aus dem transformativen Prozess heraustreten sollte, um diesen zu reflektieren, um die Unterscheidung von Forschung zu den Tätigkeiten der Zivilgesellschaft aufrecht zu erhalten (I1), so wurde doch genau diese aktive Rolle als wichtig beschrieben, da diese von niemand anderem eingenommen würde:

„Und da zeigt ja einfach die Erfahrung, dass alleine das Erstellen [eines Indikatorensets] einfach nicht reicht. Es wäre sicherlich denkbar, dass andere Akteure das machen. Intermediäre Akteure, Journalisten, Wissenschaftsjournalisten, die dann diese Arbeiten aufgreifen und auf konkrete Prozesse beziehen. Aber realistisch ist es ja einfach, dass das nicht stattfindet. Genau wie in der Klimawissenschaft, wo Klimawissenschaftler immer aktivistischer geworden sind, weil sie gemerkt haben: Es bringt nichts, das nur ins Journal zu schreiben. Genauso ist es auch da einfach klar, dass die Wissenschaft da einfach ihr Wissen aktiver transportieren muss.“(I2) 
Auch von den meisten externen Personen wurden beide Seiten der Tätigkeiten der Wissenschaft beschrieben (I6, I8-13) und größtenteils beide positiv bewertet. Einerseits wurden die Wissenschaftler*innen als Akteur*innen wahrgenommen, die die Indikatoren entwickeln, den Wohlstand messen und dabei anwendungsorientiert forschen (I6, I11-12), andererseits aber auch mit ihren praktischen Tätigkeiten: als Impulsgeber*innen und Berater*innen, die neue Ideen in die Stadt bringen und Veränderungen anstoßen (I5, I9, I12, DI13, DI17, DI37). Ein Interviewter der Stadtverwaltung formulierte den Vorteil der Wissenschaft als Impulsgeberin folgendermaßen:

\begin{abstract}
„Also von städtischer Seite wäre jetzt keiner auf so eine Idee gekommen. Ja weil es einfach hier so ein bisschen praktischer am Alltagsgeschäft orientiert ist und ich würde sagen, dass wirklich nur, ja was heißt nur, aber ja dass die Wissenschaft da Impulsgeber für so neue Modelle ist und die Stadt das dann eventuell aufgreift.“ (I5)
\end{abstract}

Die Wissenschaftler*innen wurden also neben der Rolle als Forschende auch im Sinne von institutionellen Unternehmer*innen (Campbell 2004, S. 177 f.) oder epistemischen Gemeinschaften (Campbell 2004, S. 106 f.; Haas 1992, S. $27-$ 29) angesehen, die neue Ideen voranbringen und Politik durch ihre Expertise unterstützen. Allerdings ist zu beobachten, dass die Wissenschaftler*innen sich meist selbst als Berater*innen in städtischen Prozessen angeboten haben und nicht aus der Stadt heraus angefragt wurden. Die Initiative ging also meist von der Wissenschaft aus (I3).

Zusätzlich wurden in diesem Fall auch die Wissenschaftler*innen selbst im Sinne von Vermittler*innen (Campbell 2004, S. 104 f.) beschrieben, die ihre Ideen von einem Netzwerk in ein anderes vermitteln und ihre Ideen an die Öffentlichkeit bringen. So beschrieben einige Interviewte sie als Netzwerker*innen (I10-12). In dieser Rolle wurden insbesondere die Leitungen der beiden Institutionen und des Projektes genannt, die in der Stadt gut vernetzt und anerkannt sind (I1-4, DI2931, DI40). Dazu zählen beispielsweise ein regelmäßiger Austausch zwischen dem Präsidenten des WI und dem Oberbürgermeister ${ }^{3}$ (I13) sowie auch die langjährige Zusammenarbeit auf unterschiedlichen Ebenen zwischen Universität und Stadt (I7). Im Laufe des Projektes wurde außerdem ein wissenschaftlicher Beirat des Oberbürgermeisters gegründet, in dem auch die Projektleitung vertreten ist, was die Vernetzung mit der Stadt und anderen Akteur*innen noch intensivierte (I1). Daneben hatten die Projektveranstaltungen einen Netzwerkeffekt innerhalb der Gruppe der Nachhaltigkeitsakteur*innen, der von einigen aus der Zivilgesellschaft

\footnotetext{
${ }^{3}$ Diese und folgende Funktionsbezeichnungen beziehen sich jeweils auf die personelle Besetzung zum Zeitpunkt der Interviews.
} 
als positiv hervorgehoben wurde (I10-12). Ein Interviewter der Zivilgesellschaft beschrieb die Netzwerkfunktion und das Engagement der Wissenschaftler*innen:

„Sicherlich sehr gut und relevant ist, dass die Wissenschaftler hier vor Ort ansässig waren und sind und auch eben viele Akteure kennen und Anknüpfungspunkte haben in der Stadtgesellschaft.“ (I9)

Auch die Einladung eines Projektmitarbeiters zu den Treffen des Lenkungskreises W2025 intensivierte den Austausch zwischen Stadt und Wissenschaft (I7-8, I13). Durch diese verschiedenen Prozesse sowie die Stellung, die Universität und WI bereits genossen, kann die Voraussetzung von Politikwandel, dass die institutionellen Unternehmer*innen über Netzwerke verfügen, insgesamt als vorliegend beobachtet werden (Campbell 2004, S. 178, siehe Abschn. 3.3.2).

Zusammenfassend lässt sich also festhalten, dass die Wissenschaftler*innen neben der Rolle als institutionelle Unternehmer*innen außerdem noch als Vermittler*innen agierten. Beide Funktionen wurden von den Interviewten als positiv wahrgenommen und die Wissenschaftler*innen als epistemische Gemeinschaft anerkannt. Ihre hohe Reputation und Glaubwürdigkeit erleichterten so den Zugang zu den Netzwerken und Entscheidungsträger*innen.

\subsubsection{Rahmenbedingungen}

Für den Erfolg institutioneller Unternehmer*innen bei der Umsetzung ihrer Ideen spielen außerdem Rahmenbedingungen wie Probleme und Widersprüche (Blyth 2002, S. 10 f.; Campbell 2004, S. 174 f.) sowie Veränderungen von Machtkonstellationen (Quack 2006, S. 180) eine Rolle, weshalb diese im folgenden Abschnitt dargestellt werden.

In den Interviews und Dokumenten wurden verschiedene Rahmenbedingungen genannt, unter denen das Projekt stattgefunden hat. Einige davon können im Sinne von Hay (2006, S. 67) als Krisen verstanden werden, die als solche wahrgenommen und kommuniziert werden und neue Lösungen erfordern (siehe Abschn. 3.3.2). So wurden insbesondere die hohe Verschuldung der Kommune und die schlechte wirtschaftliche Lage infolge des Strukturwandels in vielen Interviews sowie in zahlreichen Dokumenten beschrieben (I1-4, I7-9, I11, DI2, DI5-6, DI10-11, DI30, DI38, DI40).

„Das größte Problem der Stadt ist das Schuldenproblem. Die Stadt ist überschuldet und der Schuldenberg ist so groß, dass die Stadt das nicht aus eigener Kraft lösen kann 
und dadurch sind erstmal die Gestaltungsmöglichkeiten der Stadt von vorne herein auf unbegrenzt lange Zeit massiv eingeschränkt. So, das ist ein großes Problem.“ (I9)

Die Interviewten beschrieben dies als Herausforderung, die auch in der Stadtgesellschaft als solche wahrgenommen und kommuniziert wird. Aufgrund der hohen Schulden wurde oft der mangelnde Entscheidungsspielraum der Kommune als negativer Punkt genannt (I1-4, I11) und an vielen Stellen wurde derart gespart, dass nun einige Prozesse in der Verwaltung nicht mehr wie gewünscht funktionierten (I8, I11). Zusätzlich wurden ein Mangel an Arbeitsplätzen (I1, I4), eine hohe Armutsquote (I1, I4-5, I10, I13, DI30), niedrige Bildungsabschlüsse (I5, I7, I11) sowie Ungleichheit und damit zusammenhängend eine Spaltung der Gesellschaft (I10, DI30) genannt. Zusätzlich sieht sich Wuppertal mit Herausforderungen in Bezug auf die Integration von Geflüchteten und anderen Migrant*innen konfrontiert (I1, I7, I10, DI6). Auch die Themen Bildung und Armut wurden von den Interviewten als offen kommunizierte Krisen angesehen (I5-7, I9, I13). Daneben leidet Wuppertal unter einem schlechten Image und Selbstbild der Stadt, und schneidet auch in Städterankings meist schlecht ab (I1-2, DI10). An diesen Rankings kritisierte ein Interviewter, dass die negative Bewertung niedriger Mieten im Gegensatz zu den positiven Effekten für Niedrigverdienende stehe. Hier liegt also ein Widerspruch vor zwischen der negativen Bewertung in Rankings und dem auch dadurch schlechten Image der Stadt auf der einen Seite und dem eigentlich positiven Aspekt niedriger Mietkosten für die Bevölkerung auf der anderen Seite (I2).

Des Weiteren gibt es eine hohe Leerstandsquote und heruntergekommene Straßenzüge (I1, I10, DI10, DI30-31). Damit zusammenhängend nannte ein Interviewter energetische Sanierung, Wohnen im Alter sowie den Erhalt des Gebäudebestandes als Herausforderungen (I10). Ein anderer erwähnte die Herausforderung, Wohnraum zu schaffen (I13). Weiterhin wurde das Thema Mobilität genannt, beispielsweise der Wunsch, den ÖPNV zu verbessern und auch das Thema Verkehrslärm (I9-11). Ein Interviewter nannte außerdem den Widerspruch zwischen verschiedenen Entwicklungspfaden der Stadt, einerseits alternative Projekte zu unterstützen und andererseits investorengetriebene Entscheidungen zu treffen (I2). Auch glaubte ein Interviewter, viele Verwaltungsangestellte würden gerne nach Nachhaltigkeitskriterien entscheiden, seien aber oft an wirtschaftliche Aspekte gebunden (I11). Die Interviewten sahen noch weitere Widersprüche, wie zum Beispiel zwischen ökonomisch motivierten Entscheidungen und den ökologischen und teilweise auch ökonomischen negativen Folgen (I1). Neben diesen speziell in Wuppertal vorhandenen Rahmenbedingungen und Krisen wurden auch einige Themen genannt, die über die Stadtgrenzen hinausreichen. So wurden der 
Klimawandel und die Übernutzung von Ressourcen als Bedrohung beschrieben, die die Notwendigkeit einer Nachhaltigkeitstransformation mit sich bringt (I1, I9, I11-12, DI2, DI11, DI18, DI32, DI36, DI38-40). Dafür seien neue Instrumente und neue Werte notwendig (I9) und neue Gewohnheiten in der Gesellschaft:

\begin{abstract}
„Also die Nachhaltigkeit in die Stadt bringen und so, dass sie eben Spaß macht. Dass es auch eine Selbstverständlichkeit wird. Es darf nicht mehr so einfach sein, das Verkehrte zu tun [...]. Es muss in Fleisch und Blut übergehen. Andere Sachen sind ja auch einfach normal. Wenn ich über die Straße gehe, dann gucke ich auch vorher rechts und links, weil ich weiß, das gefährdet mein Leben. Aber mit dem Klimawandel gehe ich einfach so locker um, als ob der mich gar nicht angeht."(I11)
\end{abstract}

Das Thema Nachhaltigkeit wurde hauptsächlich von den institutionellen Unternehmer*innen und den Mitgliedern zivilgesellschaftlicher Gruppen, nicht jedoch von den Entscheidungsträger*innen genannt. Dieses sowie weitere genannte Herausforderungen stellen daher keine Krisen dar, die zu einem Hinterfragen bestehender Programme und Paradigmen führten. Die schlechte wirtschaftliche Lage und die daraus resultierenden hohen Schulden der Kommune, die hohe Armutsquote und die Notwendigkeit einer Verkehrswende wurden dagegen öffentlich als Krisen wahrgenommen und kommuniziert, die neue Lösungen erfordern (Hay 2006, S. 67).

\begin{abstract}
„Also wenn ich rumfrage zum Beispiel nach dem größten Problem der Stadt, ist das eigentlich fast immer bei den Leuten im Kopf, dass das das Schuldenproblem ist, dass Mobilität ein Problem ist auch und eigentlich auch, dass es viele arme Leute gibt in der Stadt und viel Armut, insbesondere bei den Kindern, das wird auch seit Jahren immer wieder in der WZ zum Beispiel und in der Rundschau gebracht. Also das sind keine Sachen, die verdeckt sind, sondern da steht die Stadt schon dazu, dass das Herausforderungen sind, an denen man wirklich hart arbeiten muss. Und die nicht leicht zu lösen sind.“(I9)
\end{abstract}

Die Themen Verschuldung, Mobilität und Armut wurden auch von Entscheidungsträger*innen, deren Wahrnehmung und Kommunikation eines Problems eine besondere Rolle für die Chance eines Wandels spielt (Campbell 2004, S. 115, siehe Abschn. 3.3.2), beschrieben und könnten daher für die Durchsetzung neuer Ideen geeignete Anknüpfungspunkte darstellen. Bei den weiteren oben genannten Themen stellen die Chancen sich als schwieriger dar, da die Entscheidungsträger*innen diese nicht klar als Krisen definieren und davon auszugehen ist, dass sie auch ihre Macht und Ressourcen davon nicht gefährdet sehen. 
Des Weiteren beschrieben die Interviewten einige Veränderungen, die während oder vor dem Untersuchungszeitraum stattgefunden haben und die die Verbreitung und Umsetzung der Ideen möglicherweise beeinflusst haben. Auch wenn hier einige Punkte aus Abschnitt 2.4 wieder aufgegriffen werden, stellt dies nicht lediglich eine Wiederholung dar, da es an dieser Stelle darum geht, welche Veränderungen die interviewten Expert*innen - nicht die Autorin - mit den Ideen des ersten Falles in Verbindung bringen. So wurde der Umbau des Bahnhofsvorplatzes Döppersberg bereits einige Jahre zuvor begonnen und von vielen als negativ wahrgenommen (I1, I11-12). Während des Untersuchungszeitraumes wurden dann die Flüchtlingswelle 2015 (I1, DI18), die Gründung des Dezernats für Bürgerbeteiligung, heute Stabsstelle Bürgerbeteiligung, beobachtet und als relevant angesehen (I2, I9, DI30). In diese Zeit fielen außerdem die Wahl eines neuen Oberbürgermeisters (I4, DI30) und die Gründung des wissenschaftlichen Beirates des Oberbürgermeisters (I1). Nach der Oberbürgermeisterwahl entstand die Konstellation einer stabilen Mehrheit einer Koalition aus SPD und CDU im Stadtrat mit gleichzeitig einem Oberbürgermeister, der für viele Entscheidungen keine Mehrheit hinter sich hatte (I2, I11). Außerdem fand eine Landtagswahl mit anschließendem Regierungswechsel statt (I4). Zusätzlich gab es einige Prozesse auf der Stadtebene: Der Stadtentwicklungsprozess W2025 war auf der Suche nach einem Maßstab zur Evaluierung der 13 enthaltenen Projekte (I2, I7, I13, DI12, DI13), mit der Entwicklung der neuen Stadtentwicklungsstrategie STEK2030 wurde begonnen (I4), das erste Bürgerbudget wurde durchgeführt und benötigte dafür geeignete Gemeinwohlkriterien (I11, DI17, DI20). Die Offene-Daten-Strategie der Stadt Wuppertal wurde umgesetzt und das Thema gewann an Bedeutung (I2). Besonders die institutionellen Veränderungen durch die neue Stabsstelle Bürgerbeteiligung, dadurch vermehrte personelle Ressourcen in dem Bereich sowie die Stadtentwicklungsstrategien könnten den Erfolg der Projektideen vorangebracht haben. In beiden Bereichen waren die institutionellen Strukturen noch nicht verfestigt und insofern noch offener für neue Impulse.

In Bezug auf die Forschungslandschaft in Wuppertal gab es vor Projektbeginn Veränderungen, die eine Rolle gespielt haben und von den Interviewten erwähnt wurden. So wurde Uwe Schneidewind 2010 als Präsident des WI ernannt und brachte neue Schwerpunkte in die Forschung zur Transformation in Wuppertal (I1). Außerdem wurden zahlreiche zivilgesellschaftliche Initiativen in verschiedenen Quartieren in Wuppertal sowie 2013 das Transzent gegründet (I1).

Aus den Interviews lässt sich schlussfolgern, dass insbesondere die neuen institutionellen Strukturen wie die Stabsstelle Bürgerbeteiligung, der neue Oberbürgermeister sowie die Stadtentwicklungsstrategien institutionelle Kontexte darstellten, 
an die die Ideen des Falles gut anknüpfen konnten. Die Herausforderungen durch die hohen Schulden der Stadt und dem damit zusammenhängenden schlechten Image stellen möglicherweise eine Situation dar, die neue Lösungen von institutionellen Unternehmer*innen erfordert.

\subsubsection{Anknüpfungspunkte und Umsetzbarkeit der Ideen}

Voraussetzung für erfolgreiche Verbreitung und Umsetzung von Ideen ist es, an die vorhandenen wahrgenommenen Krisen oder Widersprüche mit Lösungsvorschlägen anzuknüpfen und diese als relevant, geeignet und umsetzbar darzustellen (Campbell 2004, S. 177 f., siehe auch Abschn. 3.3.2). Im Folgenden wird daher gezeigt, wo dies gelang und wo die Anknüpfung sich schwieriger gestaltete.

Die interviewten institutionellen Unternehmer*innen beobachteten eine positive Einstellung der Entscheidungsträger*innen gegenüber dem Projekt (I2-3) und auch von diesen wurde in den Interviews das Projekt als relevant beschrieben (I5-7, I13). Trotzdem hat sich bisher keiner der Entscheidungsträger*innen aktiv für die Ideen eingesetzt, außer der bereits genannten Person eines städtischen Betriebes, die als Vermittlerin agierte.

In einer Stadt mit überwiegend negativem Image waren die zwölf Dimensionen vor allem dann gern gesehen, wenn sie dazu dienten, die positiven Seiten der Stadt darzustellen. Hier kann die Idee des Projektes also an eine wahrgenommene Krise anschließen, indem sie zeigt, dass mehr als nur die Finanzlage die Lebensqualität in Wuppertal ausmacht und auf diese Weise zu einer Verbesserung des Images beitragen (I2, I4, I6-8, I13, DI2). Außerdem ist die Stadt durch den Mangel an Ressourcen interessiert daran, Synergien zu nutzen, indem auf Ergebnisse zurückgegriffen wird, die von der Wissenschaft aus deren finanziellen Mitteln entwickelt wurden (I9). Ein Mitarbeiter der Stadtverwaltung formulierte die Relevanz der Projektideen für Stadtentwicklung wie folgt:

„Halte ich auch für sehr wichtig. Weil es in der Stadt, gerade in den letzten zehn, fünfzehn Jahren eben immer sehr um den finanziellen Aspekt kreist; unter dem Stichwort ,Kommune, die sparen muss und kein Geld hat' und man auf der anderen Seite eben sieht, dass es eine extrem kreative Stadt ist. Also jetzt gerade auf Wuppertal bezogen, mit viel Engagement, wo Leute eben auch was für diese Stadt tun und... oder für ihre Gemeinschaft tun. Und dadurch merkt man: Okay, das ist denen wichtig und die wohnen gerne hier und es kann nicht an ökonomischen Faktoren liegen. [...] Aber eigentlich deshalb umso wichtiger, dass man eben auf, auf solche Indikatoren auch zurückgreifen kann, um vielleicht auch nicht immer diesen... dieses Spardiktat irgendwie nach vorne zu stellen. Sondern auch zu merken, hier... es geht auch darum, 
dass ich andere Möglichkeiten der Beteiligung oder des kulturellen Zusammenlebens schaffe, die einen unheimlichen Boost auf, auf ein Wohlbefinden geben können. Und dementsprechend da auch strategisch vielleicht näher an den wirklichen Präferenzen der Bürgerinnen und Bürger zu sein.“ (I6)

Hier wurden die Wohlstandsdimensionen als konkreter Lösungsvorschlag formuliert und auch als geeignet angesehen (I7, I9). Beim Bürgerbudget sowie auch bei W2025 konnte die Stadt auf bestehendes zurückgreifen und Synergien nutzen. Bei diesen Projekten war sie auf der Suche nach konkreten Instrumenten oder Kategorien, was die Anschlussfähigkeit erhöhte (I7-8, DI7, DI30). Die Wohlstandsindikatoren oder -dimensionen stellten ein mögliches Instrument dar und einen Vorschlag für ein gerade gesuchtes Kriterienset (I3, I6-9, I13). Dieses Zusammentreffen eines Bedarfs in der Stadtverwaltung beim Bürgerbudget mit dem Angebot der Forschenden wurde von einem Mitarbeiter aus der Stadtverwaltung folgendermaßen beschrieben:

\begin{abstract}
„Wir wollen, dass Leute Entscheidungen treffen, die auch gemeinwohlbasiert sind, um dann eben auch zu gucken, wie kann man die Arbeit, die auch bei euch in diesem Projekt Wohlstandsindikatoren gelaufen ist, auch da runter brechen. So, das war halt eine direkte Zusammenarbeit.“(I6)
\end{abstract}

Ähnlich gut konnten die Ideen des Projektes auf dieser Ebene an W2025 anknüpfen, da dort gerade ein Maßstab zur Evaluierung gesucht wurde. Ein projektinterner Interviewter resümierte, dass beim W2025-Prozess,

,[...] schon immer - bevor wir da überhaupt reingekommen sind - klar war: Um den Erfolg dieser Projekte zu messen, brauchen wir mehr als ein Wirtschaftswocheranking, brauchen wir mehr als Wirtschaftszahlen, die bewirken was Anderes. Ursprünglich sollte eine Bürgerbefragung stattfinden - regelmäßig - da war dann kein Geld dafür da. Und da quasi haben wir diese Lücke ein Stück weit geschlossen." (I2)

Zwar war es zu Beginn des Projektes bereits geplant, die Wohlstandsdimensionen an die Stadt heranzutragen, jedoch noch unklar, wie genau dies geschehen wird. Mit beiden städtischen Prozessen (W2025 und Bürgerbudget) ergab sich eine günstige Gelegenheit, die die institutionellen Unternehmer*innen nutzen, um ihre Ideen in die Prozesse einzubringen. Auf dieser untersten Ebene, der Idee, die Dimensionen in der Bürgerwerkstatt oder für Workshops mit den Reallaboren als Bewertungskriterien zu nutzen, erfordert die Umsetzung nur kleine Veränderungen (I1-4, I6-9). Als ein neues Instrument ist das Projekt als passend und 
anschlussfähig beschrieben worden und wird als geeigneter Lösungsvorschlag (Campbell 2004, S. 179; Schmidt 2002, S. 221) angesehen.

Doch auch auf dieser Ebene zeigte sich, dass es nicht allen gleich leicht fiel, die Dimensionen zur Bewertung zu nutzen. Bei der Bürgerwerkstatt gelang dies nur einigen Gruppen (DI21-28). Bei den Wirkungsabschätzungsworkshops der Reallabore des WTW-Projektes, die im Gegensatz zur Bürgerwerkstatt von Wissenschaftler*innen moderiert wurden, wurde die Nutzung der Dimensionen von den Teilnehmenden als hilfreich und gut verständlich wahrgenommen (DI29). Zur Nutzung in dem späteren Stadtentwicklungsprozess STEK2030 wurden die Wohlstandsdimensionen ebenfalls vom Leiter des Ressorts Stadtentwicklung als geeignet angesehen, dann aber nicht dafür genutzt (I13).

Die Idee eines alternativen Wohlstandsverständnisses als Leitbild für städtische Politik wurde als sehr anschlussfähig zu den Akteur*innen im Nachhaltigkeitsbereich, also einer Gruppe in der Bevölkerung, wahrgenommen (I1, I9, I11-12). Sowohl lokal als auch überregional betrachtet war sie außerdem anschlussfähig an die Debatte über Nachhaltigkeitsindikatoren (I3). Ein Interviewter aus der Zivilgesellschaft beschrieb die Relevanz der neuen Paradigmen:

\footnotetext{
„Naja, weil so wie wir unsere Gesellschaft, unser Wirtschaftssystem, unser politisches System entwickeln, geht das ganze ja den Bach runter. Also wir... Also man kann ja nicht sagen, dass wir auf einem guten Weg sind, nach meiner Interpretation. Sondern wir sägen ja den Ast ab, auf dem wir sitzen. Und das heißt, wir wenden die Instrumente, die wir haben falsch an und oder haben die falschen Instrumente. Ja, vermutlich beides. Das heißt, wir müssen das, was wir da haben sehr kritisch hinterfragen und gucken, wie können wir das besser machen. Und da gehört natürlich die Messung von Wohlstandsindikatoren gehört da wesentlich mit dazu, weil sie natürlich dazu beitragen, solche Messverfahren wie Gesellschaft selbst ihre Realität wahrnimmt und das was ihre Ziele sind und wohin sie sich entwickeln kann.“(I9)
}

Aus dieser Perspektive können die Indikatoren ein Lösungsweg sein, um ressourcenschonende und nachhaltige Stadtentwicklung und städtische Lebensqualität zu fördern (DI2). Als eine Umsetzungsmöglichkeit dieser tiefgreifenden Idee wurde ein Nachhaltigkeitscheck bei städtischen Entscheidungen von drei Personen der Zivilgesellschaft genannt (I9, I11-12).

Inwieweit die Ideen des Projektes an die Vorstellungen der Stadtverwaltung anschlussfähig sind, waren sich die Interviewten uneinig. Einige meinten, das Projekt würde an die Vorstellungen vieler Verwaltungsmitarbeiter*innen anknüpfen (I11-13), jedoch nicht an die der leitenden Ebene (I11). Auch wurde es eher als Kritik an aktuellen Entscheidungen verstanden (I1-2, I5) und würde daher eine größere Veränderung fordern (I1-2, I4-5). Dass nur bei drei externen 
Interviewten - alle Nachhaltigkeitsakteur*innen der Zivilgesellschaft - die Verbreitung alternativer Paradigmen und ein Hinterfragen von Kriterien der Entscheidungsfindung als zentrales Ziel der institutionellen Unternehmer*innen ankam (19, I11-12), verdeutlicht, dass hier die Anschlussfähigkeit an den Rest der Akteur*innen sehr gering war. Daneben zeigt dies, dass es sich als wesentlich schwieriger herausstellte, dieses alternative Paradigma zu etablieren als kleine Umsetzungen in Projekten wie dem Bürgerbudget durchzuführen (I1-4).

Eine Orientierung der Stadtpolitik und -verwaltung an den Indikatoren würde Änderungen in Abläufen und Gewohnheiten bedeuten, worin einige Interviewte auch wegen knapper finanzieller und zeitlicher Ressourcen Schwierigkeiten sahen (I1-4, I6-9). Ein Mitarbeiter der Stadtverwaltung beschrieb dies wie folgt:

„Dass es zu abstrakt ist, und dass es halt in ... in ein bestehendes Umfeld aus Informationen trifft. Und das ist dann halt so: Okay, noch eine Info. So. Und das wird dann einfach in Zeiten der Arbeitsverdichtung oder auch vielleicht in einer politischen Kurzfristigkeit sagt man: Ja, ist zur Kenntnis genommen. Dann war es das. Das ist halt die Gefahr.“ (I6)

Insofern müssten die Indikatoren einfach anwendbar und nicht zu komplex sein, um in der Verwaltung etabliert zu werden (I5, I12). Ein Mitarbeiter der Stadtverwaltung merkte außerdem an, dass ein Vergleich mit anderen Städten notwendig sei und er die Indikatoren ungern als erste und einzige Stadt nutzen würde (I7). Wie von Campbell (2004, S. 179) argumentiert, hätte es hier geholfen zu zeigen, wenn diese Indikatoren bereits an anderen Orten nützlich und geeignet waren (siehe Abschn. 3.3.2). Andere dagegen sahen gerade den Wert darin, dass die Indikatoren in und für Wuppertal entwickelt und daher genau passend seien (I11).

Die Analyse hinsichtlich der Anschlussfähigkeit und Umsetzbarkeit der Ideen hat also gezeigt, dass das neue Paradigma hauptsächlich an die Nachhaltigkeitsakteur*innen, also eine Nische in Wuppertal, anschlussfähig ist, von diesen jedoch als sehr relevant angesehen wurde. Der Großteil der Bevölkerung und der Entscheidungsträger*innen sah hier dagegen weniger Bezug zu ihren Zuständigkeiten und sah die Umsetzung als zu schwierig an. Hier wurde also bisher kein genügender Handlungsdruck durch die Krisen wahrgenommen und die Ideen nicht als geeignete Lösungsvorschläge bewertet. Auf der Ebene von Policies wurde dagegen von allen Interviewten und in den Dokumenten eine Umsetzbarkeit gesehen. In einigen Fällen konnten hier sogar Ressourcen auf Seiten der Stadt gespart werden, was die Umsetzung der Policies für die Stadtverwaltung noch erstrebenswerter machte. 


\title{
5.1.4 Beobachtbare Veränderungen
}

Die Interviewten beobachteten, dass einige Akteur*innen in der Stadt vermehrt die Begriffe gutes Leben und Wohlstand im erweiterten Sinne nutzen, was als eine erste beobachtbare Veränderung verstanden werden kann. So verwendete unter anderem der Oberbürgermeister diese Begriffe sowie die Stadtsparkasse in ihrem Kundenmagazin Treuewelt (I1-2). Im Hinblick auf das bisher oft negative Image der Stadt meinte eine Interviewte, bereits eine diskursive Veränderung zu beobachten:

\begin{abstract}
„Und in diesem Zusammenhang denke ich, haben wir es wirklich geschafft, einen Beitrag dazu zu liefern, zu sagen, Mensch, es gibt auch Sachen, da sind wir wirklich gut drin'. Ja, o. k., wir haben wirtschaftliche Probleme, Bevölkerungsrückgang. Aber wir haben beispielsweise zivilgesellschaftliche Initiativen, die ganz stark zum Wohlstand beitragen in dieser Stadt. Und diesen Negativdiskurs umzudrehen ein Stück weit zu einem positiven Diskurs. Und ich denke auf dieser diskursiven Ebene, haben wir einiges geschafft.“(I1)
\end{abstract}

Bei den beiden Prozessen Bürgerbudget 2017 und W2025 entschieden sich externe Personen bewusst für die Nutzung der Wohlstandsdimensionen und damit wurde die Idee ein erstes Mal aktiv unterstützt (I7-8, DI13, DI17, DI32). Bei der Durchführung des Bürgerbudget 2017 wurden die Wohlstandsdimensionen dann auch bereits genutzt, wohingegen bei W2025 noch nicht mit der Nutzung begonnen wurde und noch nicht geklärt ist, wie dies geschehen soll (I8). Auch wenn die Ideen bei den meisten Entscheidungsträger*innen und zivilgesellschaftlichen Akteur*innen auf positive Resonanz gestoßen sind, haben sich bisher nur wenige Personen aktiv dafür eingesetzt oder die Indikatoren aufgegriffen. Zwei Personen nannten als Grund dafür einen Mangel an zeitlichen Ressourcen und die Vielzahl anderer Aktivitäten (I10, I12).

Die bisherigen kleinen Anwendungen der Wohlstandsdimensionen beim Bürgerbudget und in Wirkungsabschätzungsworkshops beziehen sich auf die hinter dem Projekt stehenden neuen Policies. Im Sinne der Einführung neuer Instrumente (Wandel erster Ordnung, Hall 1993, S. 278) wurde also schon ein Schritt erreicht. Die dahinterliegenden Paradigmen wurden bei den bisherigen Anwendungen jedoch nicht verändert, sind aber zu einzelnen Personen vorgedrungen und in den Diskursen verbreitet worden. 


\subsubsection{Zusammenfassung und Abgleich mit den Prognosen}

Im Folgenden wird die vorangegangene Analyse des Projektes der Wohlstandsindikatorenentwicklung im Rahmen des WTW-Projektes nun zusammengefasst und die Ergebnisse werden eingeordnet, um im Sinne einer Kongruenzanalyse die laut diskursivem Institutionalismus vorhergesagten Prognosen (siehe Abschn. 4.3) mit den Beobachtungen in der Empirie zu vergleichen.

Wie in den vorangegangenen Abschnitten beschrieben, waren neue Ideen vorhanden, die von den Mitarbeiter*innen der Forschungsinstitute WI und Transzent vorangebracht wurden. Diese Akteur*innen können daher als institutionelle Unternehmer*innen verstanden werden, die sich für diese Idee eingesetzt haben (Campbell 2004, S. 177 f.) oder auch als epistemische Gemeinschaft, die als Berater*innen herangezogen wurden (Campbell 2004, S. 106 f.; Haas 1992, S. 27-29).

Diese institutionellen Unternehmer*innen bezweckten Veränderungen auf den unterschiedlichen Ebenen. Eine Nutzung der Wohlstandsindikatoren zur Evaluierung von Projekten würde mit einem Wandel erster Ordnung, teilweise auch zweiter Ordnung einhergehen. Eine einzelne Anwendung der Wohlstandsdimensionen stellt als Instrument beim Bürgerbudget eine neue Policy dar, während eine Integration dessen in ein größeres stadtpolitisches Konzept oder die Zielsetzung eines - auf den Indikatoren aufbauenden - Nachhaltigkeitschecks für stadtpolitische Entscheidungen ein neues Programm darstellen würde. Eine Orientierung stadtpolitischer Entscheidungen an einem neuen Wohlstandskonzept als übergeordnetem Kompass ist dagegen als neues Paradigma (Campbell 2002, S. 22 f., 2004, S. 94 f.; Schmidt 2017, S. 251, siehe Abschn. 3.2.1) und damit als Wandel dritter Ordnung zu verstehen (Hall 1993, S. 279, siehe Abschn. 3.3.1).

$\mathrm{Da}$ es sich um ein finanziell gefördertes Forschungsprojekt handelte, waren Ressourcen für die Verbreitung der neuen Ideen vorhanden, was ebenfalls Voraussetzung für erfolgreiche Politikveränderungen ist (siehe Abschn. 3.3.2). Parallel zu den Aktivitäten institutioneller Unternehmer*innen agierten die Wissenschaftler*innen in ihrer Rolle als Forschende, indem sie untersuchten, was Wohlstand für die Wuppertaler Bevölkerung bedeutet und wie dieser gemessen werden kann.

Die Analyse hat gezeigt, dass die Ideen des Projektes zu einem gewissen Teil in der Stadtgesellschaft und -verwaltung diffundiert sind und dabei auch einige Unterstützer*innen für sich gewinnen konnten. Dazu waren die bestehenden Netzwerke der Wissenschaftler*innen sowie der neu gewonnen Unterstützer*innen hilfreich. Bis Ende des Untersuchungszeitraumes war das Projekt allerdings noch kaum in der Öffentlichkeit angekommen und wurde so nur von einem ausgewählten Kreis an Personen wahrgenommen. Die quantitativen Ergebnisse der 
Indikatorenberechnung sind in den öffentlichen Dokumenten nur bruchstückhaft dokumentiert. Meist wurden nur die Dimensionen ohne dazugehörige Indikatoren kommuniziert. Außerdem zeigte sich, dass die involvierten Personen meist nur einzelne Teilaspekte des Projektes wahrgenommen haben und dies nicht in Gänze überblickt haben. Auch in den Medien waren die Ideen des Projektes schwer zu vermitteln.

Dies könnte darauf hindeuten, dass die Ideen des Projektes nicht, wie von Campbell (2004, S. 177 f.) als Kriterium für erfolgreichen institutionellen Wandel formuliert, einfach und verständlich kommuniziert wurden. Die Vielschichtigkeit und teilweise fehlende Eindeutigkeit der Ideen (Schmidt 2006, S. 253, siehe Abschn. 3.2.3) konnte also kaum positiv genutzt werden, sondern stellte sich eher als Hindernis für die breite Diffusion der Idee im städtischen Diskurs dar.

Im Laufe des Projektes konnten zwei Vertreter der Zivilgesellschaft und eine Vertreterin eines städtischen Betriebes als Unterstützer*innen und Vermittler*innen (Campbell 2004, S. 104 f.) gewonnen werden, die die Projektideen in weitere städtische Netzwerke einbrachten und so den Zugang zu Entscheidungsträger*innen und die Umsetzung der Ideen förderten. Durch diese Kontakte kamen auch die bisherigen Integrationen der Wohlstandsdimensionen in städtische Prozesse zustande: Die Nutzung der Dimensionen beim städtischen Bürgerbudgetprozess 2017 sowie die Entscheidung für eine Nutzung im Rahmen der Stadtentwicklungsstrategie W2025. Zugang zu Entscheidungsträger*innen (Campbell 2004, S. 178 f.) lag also vor, wurde jedoch nur in den genannten beiden Einzelfällen intensiviert. Dieser Zugang wurde durch institutionelle Veränderungen in Wuppertal ermöglicht, die geeignete Gelegenheitsfenster öffneten. So stellten die Schaffung einer neuen Stabsstelle Bürgerbeteiligung und daraus folgende vermehrte personelle Ressourcen in diesem Bereich sowie die Stadtentwicklungsstrategie W2025 Prozesse dar, an welche die Ideen gut anknüpfen konnten. In beiden Bereichen waren die institutionellen Strukturen noch nicht verfestigt und deshalb noch offen für neue Impulse. Auch die Krisensituation durch die seit Jahrzehnten andauernde schlechte wirtschaftliche Lage der Stadt und die hohe Armutsrate schien letztlich dazu zu führen, dass viele Entscheidungsträger*innen offen dafür waren, Synergien zu nutzen und auf Ergebnisse zurückzugreifen, die in der Wissenschaft entstanden sind, um so selbst Ressourcen zu sparen. Hier wurden die Wohlstandsdimensionen als Lösungsvorschlag für fehlende Bewertungskriterien formuliert und auch als geeignet angesehen, was Voraussetzungen für erfolgreichen Wandel sind (siehe Abschn. 3.3.2). Gleichzeitig bestand ein Interesse an einer positiveren Darstellung Wuppertals, das oft durch die schlechte Wirtschaftslage ein negatives Image bekommt. Auch hier konnten die Ideen eines alternativen Wohlstandsverständnisses gut anknüpfen. Auf dieser höheren Ebene 
war zwar Anschlussfähigkeit vorhanden, die Hürden der Umsetzung waren jedoch zu hoch und die Diffusion dieses Paradigmas zu gering, so dass es bisher nicht zu einer Veränderung höherer Ordnung kam.

Zusammenfassend lässt sich feststellen, dass die Kriterien Vorhandensein institutioneller Unternehmer*innen, neuer Ideen und wahrgenommener Krisen also vorliegen, eine Anknüpfung jedoch bislang nur auf der Ebene von Policies gelang. Wie sich durch den diskursiven Institutionalismus im Voraus vermuten ließ (siehe Abschn. 4.3), war eine Umsetzung auf höherer Ebene bisher nicht erfolgreich.

\subsection{App-basiertes Panel „Glücklich in Wuppertal“}

\subsubsection{Akteur*innen und Ideen}

\subsubsection{Institutionelle Unternehmer*innen und deren Ideen}

Zur Analyse des zweiten Falles, des Projektes „Glücklich in Wuppertal“, wird nun ebenfalls untersucht, ob durch das Projekt bereits ein Wandel in Wuppertal zu beobachten ist. Zunächst wird dargestellt, welche institutionellen Unternehmer*innen im Fall von „Glücklich in Wuppertal“ vorhanden waren und ob sie über Ressourcen verfügt haben.

Das Projekt „Glücklich in Wuppertal“ wurde von vier Projektpartner*innen durchgeführt, wobei die Ideen und die Umsetzung hauptsächlich von den zwei involvierten wissenschaftlichen Instituten HRO und WI ausging. Im Laufe der Projektplanungen konnten dann zwei lokale Unternehmen - die Stadtsparkasse Wuppertal sowie die WSW - gewonnen werden (G1-4). Somit sind drei der vier Projektpartner*innen lokal angesiedelte Organisationen, eine aus der Region. Diese Konstellation aus wissenschaftlichen Einrichtungen und Unternehmenspartner*innen, jeweils gut vernetzt und mit gutem Ruf in Wuppertal, brachte dem Konsortium die notwendige Glaubwürdigkeit (G1, DG4). Externe Interviewte bewerteten diese Zusammenarbeit der wissenschaftlichen Einrichtungen mit der WSW und Sparkasse positiv, da es sich um anerkannte kommunale Unternehmen handelte. Diese Zusammenarbeit habe laut den Interviewten zu einer besseren Verankerung in der Stadt geführt und zu einer größeren Bekanntheit beigetragen als bei rein wissenschaftlich getragenen Projekten (G6-9, G12-13).

Die vier involvierten Organisationen und ihre dafür zuständigen Mitarbeitenden waren unterschiedlich stark in das Projekt involviert. Projektleitung und die meisten Personalressourcen lagen beim WI. Die HRO als zweite wissenschaftliche Akteurin lieferte Wissen in den Bereichen Glücksforschung, stellte die App 
zur Verfügung und passte sie für das Projekt entsprechend an (G2, G4). Die Unternehmen Sparkasse und WSW trugen insbesondere durch finanzielle Unterstützung zum Projekt bei, indem sie ein Spendenvolumen zur Verfügung stellten, das nach Beantwortung der Fragen in der App über die Plattform „Gut für Wuppertal“ verteilt werden konnte. Daher war zusätzlich das Unternehmen Betterplace.org involviert, über dessen Plattform das Spendenportal der Sparkasse betrieben wird (G4, DG5-6, DG13). Außerdem agierten die Sparkasse und die WSW bei der Fragebogenerstellung als Kommentatorinnen und gaben Feedback (G2-4). Zusätzlich entwickelte die Sparkasse das Design der App und warb in ihren Kanälen für die Teilnahme an den Befragungen. Dazu nutze sie ihre Newsletter sowie Sparkassenautomaten und ihr Kund*innenmagazin (G1-4, DG69). Zusätzlich wurde für eine Teilfinanzierung das FGW gewonnen, das bei den Auswertungen und Publikationen auch über die Finanzierung hinaus inhaltlich involviert war (G2).

Eine Finanzierung des Projektes konnte für drei Jahre sichergestellt werden, so dass am WI ein Projektkoordinator sowie eine studentische Hilfskraft dafür zur Verfügung standen und die HRO die App auf Wuppertal anpassen konnte. Ressourcen, die institutionellen Unternehmer*innen laut Campbell (2004, S. 178 f., siehe Abschn. 3.3.2) zur Verfügung stehen müssen, um erfolgreich Veränderungen zu bewirken, waren also zum Aufbau der App vorhanden.

Mit dem Projekt „Glücklich in Wuppertal“ wurden von den Projektpartner*innen verschiedene Ziele verfolgt, hinter denen Ideen der unterschiedlichen Kategorien nach Schmidt (2017, S. 251, siehe Abschn. 3.2.1) standen. Dabei ist deutlich geworden, dass nicht alle Projektbeteiligten dieselben Projektideen verfolgten, sondern teilweise auch nur einen Teil davon, worauf im Folgenden genauer eingegangen wird.

Ein Ziel des Projektes war es, herauszufinden, was die Menschen in Wuppertal beschäftigt und was sie glücklich macht sowie das Glücksniveau zu messen (G35, DG2-3, DG8, DG20, DG23, DG57-58, DG62, DG99, DG130). Zusätzlich sollte, um dieses Ziel längerfristig zu erhalten, ein App-basiertes Panel aufgebaut werden (DG6, DG182). Diese Ziele können als klassischer wissenschaftlicher Beitrag des Projektes verstanden werden.

Die Wissenschaft sollte außerdem aber auch aus den Ergebnissen Handlungsempfehlungen und Leitlinien ableiten und die Ergebnisse für Stadtentwicklung nutzbar machen, damit die Politik sich besser an den Wünschen der Bevölkerung orientieren kann (G2-4, DG3, DG6, DG20, DG23, DG36, DG67, DG130, DG174). Auch bei konkreten Fragen wie zum zu der Zeit diskutierten Bau einer Seilbahn könnte die App helfen, indem sie als Beteiligungsinstrument genutzt wird (G2-4, DG57). Für die Nutzer*innen sollte die App die Vorteile haben, 
einerseits über das eigene Glück zu reflektieren und andererseits, sich in Stadtpolitik einzubringen (DG4, DG8, DG55). Daneben konnten sie als Dank und Anreiz für die Teilnahme Spendenguthaben an Wuppertaler Projekte vergeben (DG8, DG56, DG99). Diese Aspekte sind auch als wissenschaftlicher Beitrag, jedoch im Sinne von Politikberatung zu verstehen, als ein Angebot an die Politik, sich mehr an den Wünschen der Wuppertaler Bürgerschaft zu orientieren

Hinter diesen Zielen stand die Idee, selbst Einfluss auf stadtpolitische Entwicklungen zu nehmen. So könnten aus den neuen Erkenntnissen neue Programme abgeleitet und eingeführt werden. „Glücklich in Wuppertal“ könnte also selbst ein neues Instrument darstellen und gleichzeitig durch die Erkenntnisse die Einführung weiterer neuer Policies bewirken. Eine Veränderung von Instrumenten und Programmen durch neue Erkenntnisse über die Wünsche der Bevölkerung würde in den meisten Fällen - beispielsweise bei der Entscheidung für oder gegen den Bau der Seilbahn - eine Veränderung erster Ordnung bedeuten und keinen tieferen Wandel voraussetzen (siehe Abschn. 3.3.1). Mit dieser Veränderungsintension gehen die Forschenden von „Glücklich in Wuppertal“ also über die klassische Rolle als Wissenschaftler*innen hinaus und agieren als institutionelle Unternehmer*innen.

Ein weiteres Ziel von „Glücklich in Wuppertal“ bezieht sich auf die Idee eines alternativen, ressourcenarmen Wohlstands in der Stadt, denn für ein vollständiges Set an Wohlstandsindikatoren für Wuppertal fehlten bisher noch subjektive Daten, welche mithilfe dieser App erhoben werden könnten (G1-4, DG1, DG4, DG6, DG20, DG174, DG182, DG210). Idee hinter dem Projekt war es daher zusätzlich, herauszufinden, wie ein nachhaltiger aber ressourcenschonender Lebensstil der Wuppertaler*innen, der trotzdem eine hohe subjektive Lebenszufriedenheit ermöglicht, aussehen kann (G1-2) und dazu subjektive Daten zu erheben. Damit angereicherte Wuppertaler Wohlstandsindikatoren sollten dann als Kompass für Stadtentwicklung und -politik dienen und den Blick von rein ökonomischen Aspekten auf subjektive Zufriedenheit und andere nicht-materielle Faktoren richten.

Mit dem Beitrag von „Glücklich in Wuppertal“ zu den Wohlstandsindikatoren wurde also ein transformativer Beitrag des Projektes angestrebt. Ziel war, ein neues Paradigma einzuführen, bei dem Stadtpolitik nicht mehr hauptsächlich nach ökonomischen Kriterien entscheidet, sondern den Blick auf weitere, teilweise subjektive Aspekte von Lebensqualität richtet (G2-3). Hier handelt es sich um ein neues Paradigma, also eine kognitive Idee im Hintergrund der Debatte. Intendiert wäre damit eine tiefgreifende Veränderung dritter Ordnung, also eine Transformation (Campbell 2002, S. 22 f., 2004, S. 94 f.; Schmidt 2017, S. 251). 
Hinter dem Projekt verbergen sich demnach Ideen auf verschiedenen Ebenen. Doch nicht alle an „Glücklich in Wuppertal“ Beteiligten vertreten alle dieser Ideen gleichermaßen. So sahen alle Projektbeteiligten die Ziele der Datenerhebung, um mehr über die subjektive Zufriedenheit zu erfahren und ein Befragungstool zu entwickeln, mit dem Bürger*innen zu städtischen Prozessen befragt werden können (G1-G5). Die grundlegendere dahinterstehende Idee einer Kritik an einer rein ökonomischen Perspektive auf Stadtentwicklung und der Weiterentwicklung des BLI-u wurde dagegen hauptsächlich von den Beteiligten des WI, weniger zentral auch von der HRO vertreten $(\mathrm{G} 1-2, \mathrm{G} 4)$, weshalb sie als die institutionellen Unternehmer*innen des Projektes angesehen werden. Die Rollen weiterer involvierter Akteur*innen werden später genauer beleuchtet (siehe Abschn. 5.2.1.2).

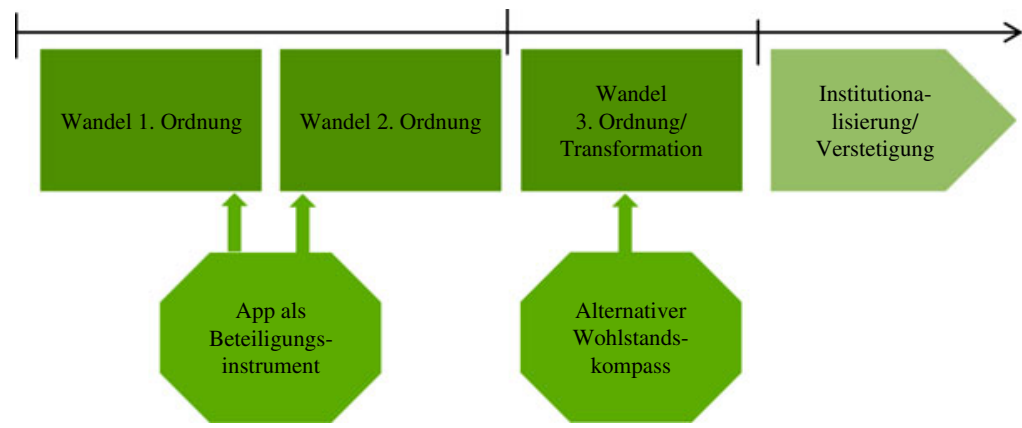

Abb.5.2 Zuordnung der Ideen zu Veränderungsintensionen (,Glücklich in Wuppertal“). Die Abbildung zeigt die Zuordnung der Ideen der institutionellen Unternehmer*innen im Fall von „Glücklich in Wuppertal“ zu den drei Graden der Veränderung nach Hall (1993). Die App als Beteiligungsinstrument zu nutzen, würde je nach Ausgestaltung einen Wandel erster oder zweiter Ordnung bedeuten, während die Einführung eines alternativen Wohlstandskompasses eine Transformation mit sich bringen würde.

Quelle: Hall (1993) und eigene. Eigene Darstellung

Die Ideen sind auf den unterschiedlichen Ebenen verortet und Veränderungsintensionen sind auf allen drei Ebenen, von einem Wandel erster Ordnung bis hin zu einer grundlegenden Transformation (siehe Abschn. 3.3.1), erkennbar (siehe Abb. 5.2). Daher konnten die Projektpartner*innen - insbesondere die Forschenden - als institutionelle Unternehmer*innen (Campbell 2004, S. 177 f.) verstanden 
werden, die ihre Ideen in städtischen Diskursen vertreten und sich für Veränderungen einsetzen. Ressourcen dafür waren für drei Jahre vorhanden und die institutionellen Unternehmer*innen genossen einen guten Ruf und Glaubwürdigkeit bei ihren Aktivitäten in der Stadt. Diese Voraussetzung für Politikwandel ist also vorhanden.

\subsubsection{Unterstützung durch zivilgesellschaftliche Akteur*innen und Unternehmen}

Um erfolgreich Wandel voranzutreiben, ist die Verbreitung der Ideen in unterschiedlichen Netzwerken notwendig und die Unterstützung durch andere Akteur*innen, die als Vermittler*innen agieren (Campbell 2004, S. 104 f., siehe Abschn. 3.3.2). Im Folgenden wird dargestellt, wer aus der Zivilgesellschaft und Unternehmerschaft in das Projekt involviert war und die Ideen des Projektes unterstützt und zu einer Diffusion beigetragen hat.

Um ihre Ideen zu verbreiten und Teilnehmende für die App zu gewinnen, pflegten die Wissenschaftler*innen Kontakt zu zivilgesellschaftlichen Akteur*innen, beispielsweise zu dem „Freien Netzwerk Kultur“ (G17) und dem Verein „Bürgerforum Heckinghausen“, in dessen Räumen eine Diskussion der Ergebnisse stattfand (G2, DG206). Auch bei „Wuppertal Aktiv“ wurde über „Glücklich in Wuppertal“ gesprochen, da es dort personelle Überschneidungen mit Entscheidungsträger*innen gab, die über andere Wege von dem Projekt erfuhren (G11, G17). Viele zivilgesellschaftliche Akteur*innen standen dem Projekt positiv gegenüber und haben die Ideen unterstützt (G2) und durch die Verbreitung in ihren Netzwerken geholfen, Teilnehmende für die Erhebungsrunden zu rekrutieren (G5).

Zwischen dem Projektkoordinator von „Glücklich in Wuppertal“ und der Wirtschaftsförderung bestand bereits Kontakt über den ersten hier analysierten Fall, das Projekt der Wohlstandsindikatoren für Wuppertal (G9, siehe Abschn. 5.1.1.2), was sich positiv auf die Verbreitung der Ideen hinter „Glücklich in Wuppertal“ auswirkte. Dadurch erfuhr die Vertreterin der Wirtschaftsförderung auch von diesem Projekt und schlug vor, die App in den Stadtentwicklungsprozess W2025 aufzunehmen, weil sie sie für ein geeignetes Instrument zur Evaluierung der Projekte hielt. Auf diesem Weg wiederum lernten zahlreiche Entscheidungsträger*innen die App kennen und die Ideen konnten auch dorthin diffundieren.

Ein Vertreter des Stadtmarketings ${ }^{4}$ erfuhr auf der Auftaktveranstaltung Genaueres über die Ideen und bot daraufhin an, darüber in seinen Netzwerken zu

\footnotetext{
${ }^{4}$ Wuppertal Marketing GmbH ist ein Betrieb, zu dessen 27 Gesellschaftern die Stadt Wuppertal, die WSW sowie die Stadtsparkasse gehören und dessen Aufsichtsrat zu einem Drittel von
} 
informieren. Er trug dadurch zu einer weiteren Diffusion der Ideen bei, insbesondere auch im Kreise von Unternehmen. Außerdem schlug er vor, die App in den Stadtentwicklungsprozess W2025 einzubeziehen (G15, G17). Persönlich fand er die Nutzung der App jedoch nach einer Weile zu zeitaufwendig und nennt im Interview einige Kritikpunkte an der konkreten Umsetzung. Trotzdem kann er als Vermittler im Sinne von Campbell (2004, S. 104 f.) verstanden werden, da er die Ideen von „Glücklich in Wuppertal“ unterstützte und sowohl in Unternehmensnetzwerke hinein als auch in städtischen Netzwerken wie dem Lenkungskreis W2025 verbreitete.

Zusätzlich bestand eine Kooperation mit der WZ, die viel über die App berichtete und in Gastbeiträgen das Thema auf eine persönliche Ebene gebracht hat, so dass es von der Leserschaft positiv aufgenommen wurde (G1-2, G6, G13, G18). Dadurch agierte die WZ als Framerin (Campbell 2004, S. 102 f.), indem sie die Programme an die Öffentlichkeit gebracht und sie so beschrieben hat, dass sie zu den Ideen der Bevölkerung passten (G1-2, G19). So berichteten die WZ-Redakteur*innen zunächst selbst in einer Kolumne über die eigene Nutzung der App (DG43, DG51, DG59, DG63, DG73, DG75-76, DG81, DG83, DG86, DG92, DG135) und luden dann Wuppertaler Persönlichkeiten aus Stadtpolitik, -verwaltung und Zivilgesellschaft dazu ein, Gastbeiträge über ihr Glück in Wuppertal zu schreiben, wodurch die WZ ebenfalls als Vermittlerin in weitere Kontexte fungierte. Dadurch wurden in der Zivilgesellschaft einige Personen und Organisationen erreicht und erfuhren über die App: zwei Kirchenangestellte (DG110, DG194), der Direktor eines Kunstmuseums (DG176), eine Galeristin (DG187), ein Mathematikprofessor (DG175), ein Lehrer (DG168), Kinder und Erzieher*innen in Kindertagesstätten (DG194, DG198, DG202), sowie ein Zauberkünstler (DG184). Diese Personen oder Organisationen der Zivilgesellschaft hatten jedoch keinen direkten Kontakt zu den Projektbeteiligten und bezogen sich in ihren Beiträgen auch nicht konkret auf „Glücklich in Wuppertal“. Es ist also nicht davon auszugehen, dass sie sich tiefgehender mit den Ideen des Projektes auseinandersetzten. Zusätzlich erfuhren über diesen Weg einige Stadtpolitiker*innen von „Glücklich in Wuppertal“ und schrieben ebenfalls Gastbeiträge in der WZ, worauf im folgenden Abschnitt zu Entscheidungsträger*innen eingegangen wird. Weitere Framerin war die Sparkasse, indem sie das Design entwickelte und in ihren Medien darüber berichtete (siehe Abschn. 5.2.1.1).

der Stadt gestellt wird (Wuppertal Marketing o. J.-a, o. J.-b). Durch diese starke Verbindung mit der Stadt wird dieser hier ebenfalls als stadtnaher Betrieb eingeordnet. 
Aus Zivilgesellschaft und Unternehmerschaft bildeten sich also drei Organisationen als Unterstützer*innen heraus: die Wirtschaftsförderung, das Stadtmarketing sowie die WZ, die alle drei als Vermittler*innen im Sinne von Campbell (2004, S. 104 f.) auftraten, die die Ideen passend für die Bevölkerung übersetzten. Daneben agierten die WZ sowie die Sparkasse als Framerinnen. Die laut Campbell (2004, S. 178, siehe Abschn. 3.3.2) für erfolgreiche Veränderung notwendigen Netzwerke der institutionellen Unternehmer*innen in unterschiedlichen Kreisen konnten so im Laufe des Projektes auch in die Zivilgesellschaft, Bürgerschaft und Unternehmerkreise herein ausgebaut werden.

\subsubsection{Zugang zu Entscheidungsträger*innen}

Voraussetzung für Politikwandel ist außerdem der Zugang zu und die Unterstützung durch Entscheidungsträger*innen. Je mehr Interesse Entscheidungsträger*innen an den Ideen haben, umso wahrscheinlicher wird ein erfolgreicher Wandel. Dies war auch den Projektbeteiligten klar, weshalb sie neben einer Verbreitung der Projektideen in der Zivilgesellschaft diese auch an Stadtverwaltung und -politik herantrugen. Dazu wurden gezielt Kontakte zu einer Auswahl an Entscheidungsträger*innen hergestellt, die die Projektbeteiligten als offen den Ideen gegenüber einschätzten (G1-2, G5). Außerdem wurden die Ideen an unterschiedlichen Stellen in städtische Prozesse integriert. In diesem Zusammenhang nannten drei der Projektbeteiligten die Herausforderung, einerseits als Wissenschaftler*innen neutral zu bleiben und nicht zum Projekt einer bestimmten politischen Gruppe zu werden und andererseits Entscheidungsträger*innen anzusprechen und für sich zu gewinnen (G1, G4-5).

Bei „Glücklich in Wuppertal“ bestand insbesondere über den STEK2030Prozess Kontakt zu Mitarbeitenden aller städtischen Ressorts sowie stadtnahen Betrieben (G1-2, G4, G6-8, G14-15, G20, DG215, DG208): das Ressort Stadtentwicklung, das den STEK2030-Prozess steuerte sowie die im Planungsgremium vertretenen Personen aus den Bereichen Gleichstellung, Klimaschutz, Grünflächen und Forsten, Straßen und Verkehr, Bauen und Wohnen, Umweltschutz, Sozialamt, Zuwanderung und Integration, Jugendamt, Kultur und Sport, Kämmerei, die Stabsstelle Bürgerbeteiligung sowie die Wirtschaftsförderung und das Jobcenter. Personen aller dieser Bereiche erfuhren so von den Ideen der institutionellen Unternehmer*innen. Der engere Austausch zwischen den Projektbeteiligten von „Glücklich in Wuppertal“ und dem STEK2030-Prozess lief dann jedoch hauptsächlich über ein von der Stadt beauftragtes Planungsbüro und teilweise über das Ressort Stadtentwicklung (G6-7, G11-12, G14). Dadurch haben die meisten Beteiligten nur wenig über die Ideen erfahren und sich nicht vertieft damit 
beschäftigt (G8, G10). In diesem Stadtentwicklungsprozess wurde die App dann das erste Mal als Beteiligungsinstrument genutzt.

Zusätzlich dazu wurde die App in den dem STEK2030 vorangehenden Prozess W2025 integriert, nachdem Vertreter*innen der Wirtschaftsförderung (G2, G8) sowie des Stadtmarketing (G1, G17) sich dafür eingesetzt hatten (siehe Abschn. 5.2.1.2). Dadurch diffundierte ein Teil der Ideen zu den anderen Mitgliedern des Lenkungskreises W2025 (G2, G8-9, G11, G14-15, G17, DG80). Die Treffen des W2025-Kreises wurden aber als sehr oberflächlich beschrieben, da aus Mangel an Zeit meist nicht vertieft diskutiert wurde (G15). Der W2025-Prozess sei außerdem mit der Zeit ins Stocken geraten, so dass beispielsweise 2018 kein Treffen mehr stattgefunden habe und daher auch kein weiterer Austausch über die Projekte und die Ideen von „Glücklich in Wuppertal“. So kam es bisher auch nicht zur Nutzung der App (G17). Die meisten dort involvierten Entscheidungsträger*innen nahmen die Ideen wohlwollend zu Kenntnis, aber beschäftigten sich größtenteils nicht weitgehender damit (G9) und auch außerhalb der eigentlichen Lenkungskreistreffen fanden keine vertieften Gespräche zwischen den dort vertretenen Entscheidungsträger*innen und den institutionellen Unternehmer*innen statt (G15).

Über die Stadtentwicklungsstrategien hinaus wurde der Oberbürgermeister gezielt angesprochen, der dann auch bei einigen Veranstaltungen des Projektes anwesend war und ein Grußwort hielt (G1-4, G13-14, DG13, DG23, DG130, DG148-49, DG151, DG161, DG181, DG189, DG210). Er und seine Mitarbeiter*innen erfuhren ganz zu Beginn des Projektes von den Ideen bei einem der regelmäßigen Austauschtreffen zwischen dem Präsidenten des WI, der gleichzeitig Projektleiter von „Glücklich in Wuppertal“ war, und dem Büro des Oberbürgermeisters sowie später erneut im Rahmen des W2025-Prozesses. Der Austausch über die App war dann jedoch nach Angaben des Büroleiters des Oberbürgermeisters nur sporadisch im Rahmen von Veranstaltungen vorhanden (G14).

Weitere Entscheidungsträger*innen haben zunächst über die Presse von einigen der Ideen erfahren, indem sie Artikel gelesen oder direkt von der WZ für Gastbeiträge angefragt wurden (G18-20). So schrieben die Fraktionsvorsitzenden der SPD (G16, DG125), der CDU (G19, DG115) und Bündnis90/Die Grünen (G18, DG102, DG162), ) der Sozialdezernent (DG89), der Kämmerer (G20, DG104), ein Landtagsabgeordneter der Freien Demokratischen Partei (DG120), zwei SPD-Landtagsabgeordnete (DG127, DG131), drei Bezirksbürgermeister*innen (DG122, DG159, DG172) und eine Mitarbeiterin der Stabsstelle 
Bürgerbeteiligung (DG157) im Rahmen der Reihe je einen Gastbeitrag und wurden dadurch zumindest oberflächlich über die Ideen von „Glücklich in Wuppertal“ informiert.

Auch wenn von den interviewten Entscheidungsträger*innen selbst als Nutzen der App genannt wurde, die Wünsche und Einstellungen der Bevölkerung zu erfragen (G1, G6-7, G11-12, G14-15, G19), hat dennoch bisher keiner von ihnen die veröffentlichten Ergebnisse genutzt oder sich nach weiteren Erkenntnissen der Befragungen erkundigt (G1-2, G4, G14-16, G18, G20). Ein projektinterner Interviewter kritisierte, dass die Ansprache der Entscheidungsträger*innen zu spät geschah und die Entscheidungsträger*innen nicht in die Fragebogenentwicklung eingebunden waren. Das habe zur Folge gehabt, dass das konkrete Angebot von „Glücklich in Wuppertal“ den Entscheidungsträger*innen unklar geblieben sei (G2, G4). Auch die Interviews mit den Entscheidungsträger*innen deuten darauf hin, dass diese selbst keinen konkreten Anwendungsbezug auf ihre Arbeitsbereiche erkannten und sich deshalb nicht konkreter damit auseinandergesetzt haben (G16, G18). Außerdem schien eine engere Zusammenarbeit zwischen den institutionellen Unternehmer*innen und den Entscheidungsträger*innen durch einen Mangel an zeitlichen Ressourcen der Stadtverwaltung erschwert zu sein (G2, G4, G8). Die Projektverantwortlichen nahmen von Seiten der Entscheidungsträger*innen eher ein Interesse an Befragungsergebnissen zu aktuellen strittigen Themen wahr - wie dem Bau einer Seilbahn - und weniger an allgemeinen Aussagen zur subjektiven Lebensqualität (G1-2).

So wurden zwar keine besonders unterstützenden Entscheidungsträger*innen mobilisiert, aber es wurden auch keine wirklich kritischen Stimmen gegen die App wahrgenommen, sondern eine breite Akzeptanz (G9, G16). Lediglich zwei Interviewte bezeichneten sich als uninteressiert am Projekt und konnten sich kaum an Ideen hinter dem Projekt erinnern (G8, G19). Einige Kritikpunkte wurden in den Interviews jedoch trotzdem genannt. Es wurden Bedenken geäußert, dass voraussichtlich nicht alle Bevölkerungsgruppen gleichermaßen erreicht und zur längerfristigen Teilnahme an der App bewegt werden könnten und die Daten deshalb nicht repräsentativ seien (G6, G8, G11-12) oder sogar nur „Freaks" die App nutzen würden (G19). Andere nannten die Gefahr, dass die App Erwartungen an Stadtentwicklung wecke, die nicht erfüllt werden könnten (G7, G10). Wenn hauptsächlich Meinungsführer*innen zu Wort kämen, würde dies zu einem falschen Bild von Beteiligung führen (G15). Innerhalb der Stadtverwaltung könnten Alltagsroutinen und Gewohnheiten die Nutzung der App und der Ergebnisse erschweren (G8-9, G11, G18). So könnte auf Seiten der Stadtpolitik die Befürchtung aufkommen, sich nicht länger politisch durchsetzen zu können und Verunsicherungen entstehen (G6, G15, G17). Ein Entscheidungsträger plädierte 
daher vor allem dafür, die App zur allgemeinen Erhebung von Stimmungen zu nutzen und nicht für konkrete Abstimmungen (G6). Eine Abfrage auf einer sehr allgemeinen Ebene wurde wiederum von anderen als nicht hilfreich empfunden und konkrete Fragen befürwortet (G1-2, G16).

Zusammenfassend lässt sich also sagen, dass durch die Einbindung in Stadtentwicklungsprozesse sowie die Gastbeiträge in der WZ viele Entscheidungsträger*innen aus allen Bereichen der Verwaltung von den Ideen von „Glücklich in Wuppertal“" erfuhren. Daneben wurden Mitglieder der meisten im Stadtrat vertretenen Parteien darüber informiert. Mit vielen der Entscheidungsträger*innen bestand jedoch kein direkter Kontakt, diese erfuhren nur über eine Ansprache durch die WZ davon oder bei wenigen Sitzungen der Stadtentwicklungsprozesse. Viele haben dadurch nur oberflächliche Informationen zu dem Projekt erhalten. Dadurch und durch zu unkonkrete Angebote an die Entscheidungsträger*innen haben sich bisher nur wenige genauer damit auseinandergesetzt und die Ergebnisse bisher nicht für ihre politische Arbeit genutzt. Teilweise lagen die Ideen des Projektes, insbesondere die App als neues Beteiligungsinstrument zu nutzen, im Sinne der Entscheidungsträger*innen, da so eigene Ressourcen eingespart werden konnten. Teilweise schien es auch eher als hinderlich für Ressourcen und aktuelle Machtverhältnisse der Entscheidungsträger*innen und wurde - vermutlich auch deshalb - nicht weiterverfolgt.

Mit der Nutzung beim STEK2030-Prozess kann die App als neues Instrument bereits einen ersten Erfolg verbuchen. Insgesamt kann also die Voraussetzung für Politikwandel - dass die institutionellen Unternehmer*innen Zugang zu Entscheidungsträger*innen haben (Campbell 2004, S. 178 f., siehe Abschn. 3.3.2) als vorliegend betrachtet werden. Welche Ebenen von Ideen dabei bei den Entscheidungsträger*innen ankamen, wird in Abschnitt 5.2.1.5 genauer analysiert.

\subsubsection{4 Öffentliche Diskurse und Diffusion der Ideen}

Weiterhin wichtig für erfolgreichen Wandel ist eine Verbreitung der Ideen in den Diskursen. Daher wird im Folgenden dargestellt, wie weit die Ideen diffundiert sind und wo darüber berichtet wurde. Dabei werden sowohl die Ergebnisse der Dokumentenanalyse als auch die Aussagen aus den Interviews über die Verbreitung der Ideen dargelegt.

Die durchführenden Organisationen waren gut vernetzt und konnten so ihre Ideen verbreiten und die Netzwerke der verschiedenen Projektpartner*innen konnten sich dabei gut ergänzen. WSW, WI und Sparkasse konnten vor allem ihre zahlreichen lokalen Netzwerke nutzen. Die HRO ist mit überregionalen und internationalen Expert*innen im Bereich Glücksforschung und Panelerhebungen vernetzt. Die verschiedenen lokalen Netzwerke der anderen Partner*innen wurden 
bei der Öffentlichkeitsarbeit genutzt, wodurch viele Bürger*innen erreicht wurden (G2).

Vom WI wurden verschiedene Werbemaßnahmen durchgeführt, um Teilnehmende zu gewinnen und auf die Ergebnisse aufmerksam zu machen (G2): An Schwebebahnhaltestellen wurden Poster aufgehängt, um für die erste Befragungsrunde zu werben. In der Stadt wurden außerdem Postkarten in Cafés und anderen Einrichtungen ausgelegt, Werbung auf der projekteigenen Webseite gemacht sowie über soziale Medien und verschiedene Newsletter per E-Mail geworben (G2, DG93, DG139, DG163, DG210).

Den Prozess der Rekrutierung von App-Teilnehmenden unterstützte die Sparkasse, indem sie auf Geldautomaten Werbung schaltete. Zusätzlich informierte sie über das Projekt in ihrem Newsletter und im Kund*innenmagazin. Dadurch sind die Informationen nochmals an viele weitere Personen gelangt (G2-4, G17, DG69).

Das Stadtmarketing verbreitete Informationen über das Projekt außerdem noch über seine Newsletter und Kanäle in den sozialen Medien. Nach eigenen Angaben wurden pro Beitrag in den sozialen Medien circa 10.000 Personen erreicht. Der Verteiler des Stadtmarketings umfasst unter anderem auch lokale Unternehmen, so dass diese auf diesem Weg ebenfalls erreicht wurden (G17). Ein projektinterner Interviewter meinte jedoch, bisher kein Interesse von Unternehmen außer der Sparkasse und WSW wahrgenommen zu haben (G2).

Zusätzlich wurden öffentliche Veranstaltungen durchgeführt. Insgesamt kamen zu diesen Veranstaltungen circa 100 Personen, insbesondere aus der Bürgerschaft (G2-4, DG189). Durch die Nutzung beim STEK2030-Prozess erfuhren außerdem die ungefähr 150 Teilnehmenden einer Zukunftswerkstatt von der App (DG189). 2000 Personen nahmen insgesamt an mindestens einer der App-Befragungsrunden teil und bekamen dadurch einen Teil der hinter dem Projekt stehenden Ideen vermittelt (G2, DG204, DG210, DG213, DG163).

Auch berichtete die WZ viel über die Ideen, die Möglichkeit der Teilnahme an den Befragungsrunden und über Ergebnisse und Projektveranstaltungen (G12, G13). Zusätzlich etablierte sie für 35 Wochen die bereits genannte Kolumne zum Thema Glück, in der die Redakteur*innen der WZ sowie Gastautor*innen über ihre persönlichen Glücksmomente und Vorstellungen vom Glück schrieben. Dadurch ist ein neuer Diskurs in der Stadt entstanden, der Leser*innen und Gastautor*innen dazu verholfen hat, über ihr Glück und Zufriedenheit in Wuppertal nachzudenken (G1-2, G4-5). Ein projektbeteiligter Interviewter beschrieb im Interview, dass dadurch 
„[...] auch um die App herum so ein Diskurs und eine Perspektive auf Glück in Wuppertal entstand. Zum Teil auch bei den Assoziationen, die die Personen haben, weit weg jetzt von klassischer, subjektiver Zufriedenheitsmessung. Aber es ist auf jeden Fall eine Diskussion über Glück und Zufriedenheit in der Stadt entstanden und es war interessant zu sehen, wie viele sich damit identifizieren können.“(G1)

Allerdings merkten einige der Interviewten an, dass die WZ auch nur einen Ausschnitt der Bevölkerung erreiche (G11, G13, G17). Mehr über die Ideen des Projektes und genauere Reflexion über das eigene Glück erfuhren diejenigen, die auch an der App teilnahmen, wobei kein repräsentatives Abbild der Bevölkerung erreicht wurde und die bereits in der Stadt engagierten Personen eher teilnahmen als andere. Es nahmen prozentual weniger Menschen mit Migrationshintergrund teil als in der Stadtbevölkerung vorhanden und auch sehr junge und sehr alte Menschen waren unterdurchschnittlich vertreten (G2, G9, G12, DG139, DG204).

Insbesondere zur Rekrutierung von Teilnehmenden wurde viel über das Projekt berichtet. Für die Dokumentenanalyse konnten 216 Dokumente gefunden werden, die im Folgenden im Hinblick auf ihre Kanäle der Verbreitung, die Autor*innenschaft, den Kontext sowie die Zeiträume der Veröffentlichung dargestellt werden, um zu untersuchen, inwieweit und wohin die Ideen von „Glücklich in Wuppertal" diffundiert sind.

In 75 der Dokumente wird das Projekt nur am Rande erwähnt; so beispielsweise in den Gastbeiträgen von Wuppertaler Persönlichkeiten in der WZ. Diese Artikel sind jeweils mit dem Logo von „Glücklich in Wuppertal“ gekennzeichnet, beziehen sich aber ansonsten kaum oder gar nicht auf das Projekt, hängen also nur indirekt damit zusammen. Die Mehrheit der Dokumente (141) dreht sich jedoch hauptsächlich um die Ideen von „Glücklich in Wuppertal“.

Die meisten der Dokumente sind öffentlich verfügbar, so sind nur acht intern und nicht veröffentlicht. Kanäle der Verbreitung waren dabei vor allem soziale Medien, wie Facebook (84 Beiträge) und Twitter (41 Beiträge) sowie Lokalzeitungen (67 Artikel). Außerdem gab es einen Radiobeitrag, einen Online-Artikel auf der Seite des lokalen Fernsehsenders und drei Berichte in städtischen öffentlichen Dokumenten und Webseiten. Neben Informationen auf der Projektwebseite wurde zweimal in den Newsbereichen anderer Webseiten über die Ideen von „Glücklich in Wuppertal" berichtet, zweimal in wissenschaftlichen Zeitschriftenartikeln, in einer Broschüre, einmal im Kund*innenmagazin der Sparkasse sowie zweimal auf einem Blog der Forschungsinstitute. Zielgruppe ist in den meisten Fällen die Bürgerschaft Wuppertals, was zu erwarten war, da die App auf 
die Teilnahme zahlreicher Bürger*innen angewiesen war. Elf Dokumente richten sich jedoch auch an die Wissenschaft, Wissenschaftspolitik oder Förderer von wissenschaftlichen Projekten.

Zeitraum der Erstellung von Dokumenten ist vor allem die zweite Hälfte des Untersuchungszeitraumes. Auf 2015 und 2016 sind jeweils lediglich drei Dokumente datiert. Im ersten Halbjahr 2017 wurden dann bereits 63 Dokumente verfasst. In diese Zeit fiel auch der erste Befragungszeitraum, so dass die meisten Beiträge potenzielle Teilnehmende zu rekrutieren versuchten. Im zweiten Halbjahr 2017 wurden sogar 114 Beiträge verfasst. 2018 entstanden im ersten Halbjahr nochmals 33 Dokumente. Die Zeit, in der die meisten Dokumente verfasst und veröffentlicht wurden, fällt in die Zeit der ersten beiden Befragungswellen, während denen jeweils circa 70 Dokumente verfasst wurden. Zusammen mit der weiteren Werbung insbesondere während der ersten Runde konnten hier auch mit Abstand am meisten Teilnehmende gewonnen werden. So konnten für die erste Welle 1103 Fragebögen ausgewertet werden, für die zweite 564 und für die beiden folgenden mit 359 und 252 Fragebögen deutlich weniger.

Die meisten Dokumente wurden von Projektmitarbeitenden verfasst, einige von projektnahen Personen, beispielsweise von Institutsmitgliedern auf ihren privaten Benutzer*innenkonten in sozialen Medien. 95 Dokumente wurden von externen Personen verfasst. Hier handelt es sich mehrheitlich um Journalist*innen, jedoch auch um die Gastautor*innen in der Lokalzeitung, von denen einige gleichzeitig städtische Mitarbeiter*innen oder Stadtpolitiker*innen sind. Diese mit eingerechnet wurden 16 Dokumente von Personen aus Stadtpolitik und verwaltung verfasst. Daneben verweisen einige Facebooknutzer*innen aus der Bürgerschaft auf die Möglichkeit der Teilnahme an der App.

Im Laufe der drei Jahre gab es viel Presseberichterstattung und zahlreiche Hinweise in sozialen Medien über die Ideen von „Glücklich in Wuppertal“. Ein großer Teil dieser Berichte ist von den Projektbeteiligten selbst geleistet worden, aber auch die Presse und andere Akteur*innen haben die Befragungsrunden der App zum Anlass genommen, über Glück und über das Projekt zu berichten. Die Dokumente in den sozialen Medien sind meist sehr kurze Ankündigungen oder Hinweise, die nur einen sehr geringen Informationsgehalt haben, jedoch das Projekt und das Thema Glück allgemein in Erinnerung rufen.

Durch die in den vergangenen Absätzen genannten Berichte sind die Ideen des Projektes sehr weit in der Stadt diffundiert, sowohl in Richtung Bürgerschaft und Unternehmen, als auch zur Stadtpolitik und -verwaltung. Dies ist insbesondere durch das Interesse der WZ am Thema geschehen. Die Öffentlichkeitsarbeit des WI in den sozialen Medien sorgte außerdem dafür, dass bereits mit dem 
Projekt in Kontakt stehende Personen regelmäßig Informationen über die Erhebungen und die Ergebnisse bekamen. Die meisten Personen erfuhren jedoch nur oberflächlich über die Ideen von „Glücklich in Wuppertal“ und die Möglichkeit der Teilnahme an der App. Tiefergehende Informationen wurden dann an einen kleineren Kreis der Teilnehmenden bei Veranstaltungen des Projektes und des STEK2030-Prozesses kommuniziert.

\subsubsection{Kommunikation der Ideen}

Nachdem untersucht wurde, wo über "Glücklich in Wuppertal“ berichtet wurde, soll nun dargestellt werden, welche der Ideen bei den erreichten Personen auch angekommen sind, auf welchen Ebenen der Ideen die Kommunikation also erfolgreich war. Dabei zeigt sich, dass meist nur ein Teil der Ideen kommuniziert wurde, je nachdem, welche im jeweiligen Kontext als passend erschienen.

Von dem Interviewten der WZ wurden die Ideen auf allen drei Ebenen wahrgenommen, einerseits die Policies und Programme - Glück im Sinne von Lebenszufriedenheit im Umfeld der Stadt zu messen und ein Beteiligungstool auch längerfristig zur Verfügung stellen, um Stadtpolitik stärker daran zu orientieren - und andererseits die übergeordneten Paradigmen - herauszufinden, was Menschen über ökonomische Faktoren hinaus glücklich macht und dafür einen alternativen Maßstab zu entwickeln, der Leitbild für städtische Entscheidungen sein soll (G13). In der Berichterstattung wurden dann aber vor allem persönliche Geschichten und Wuppertal-bezogene Aussagen kommuniziert (G2, G4-5, G8, G13-14, G18). Das positiv besetzte Thema Glück kam gut an und wurde daher von der Presse aufgegriffen (G4, G7, G9, G18). Nur wenige Zeitungsartikel erwähnten die Idee, alternativen Wohlstand in der Stadt zu etablieren, indem subjektive Daten erhoben werden (DG35, DG52, DG181, DG205).

Als Ziele von „Glücklich in Wuppertal“ wurden in der Presse und in Beiträgen von Bürger*innen in sozialen Medien vor allem formuliert, herauszufinden, was die Menschen in Wuppertal beschäftigt und was sie glücklich macht sowie das Glücksniveau beziehungsweise die Zufriedenheit zu messen (DG13, DG24, DG29, DG35, DG37, DG43, DG48, DG52, DG69, DG73, DG76, DG78, DG83, DG148, DG152, DG161, DG179, DG202, DG206, DG216). Das Ziel eines Appbasiertes Panels, um die Lebenszufriedenheit über den Zeitverlauf zu messen, wurde ebenfalls genannt (DG69, DG208). Daraus könnten Handlungsempfehlungen abgeleitet und relevante Themen identifiziert werden (DG35, DG148, DG152). Außerdem könnte es als Beteiligungsinstrument genutzt werden (DG29, DG152, DG215). Indirekt sollte dadurch dazu beigetragen werden, die Wuppertaler*innen zufriedener zu machen (DG13, DG48, DG52). Im Zusammenhang mit W2025 wurde „Glücklich in Wuppertal“ außerdem als Evaluationsinstrument für 
den Erfolg von Projekten und als Indikator für subjektive Lebenszufriedenheit beschrieben (DG80).

In kürzeren Mitteilungen in den sozialen Medien oder Erwähnungen in Zeitungsartikeln, wo das Projekt nur am Rande erwähnt wurde, wird teilweise gar nicht auf die Ideen direkt eingegangen, sondern nur erwähnt, dass dort Fragen zum Glück beantwortet und Spendenguthaben verteilt werden können (DG56, DG107, DG111). Darüber hinaus sollte die App bei der Reflexion über das eigene Glück und die eigenen Lebensumstände helfen (DG63, DG73, DG75, DG81, DG83, DG96). In der Öffentlichkeit ist so oft nur ein sehr oberflächliches Verständnis des Projektes angekommen, das sich vor allem auf die Idee der Wissensgenerierung über das Glück der Bevölkerung bezieht, also den klassisch wissenschaftlichen Teil, nicht den transformativen Anspruch. Dass wie von Schmidt (2006, S. 253) erläutert Mehrdeutigkeit in der Kommunikation von Ideen sowohl förderlich als auch hinderlich sein kann, wurde hier deutlich. So konnten mehr potenziell Interessierte erreicht werden, gleichzeitig blieben die eigentlich hinter "Glücklich in Wuppertal“ stehenden Ideen aber auch für viele unklar. Durch die vielen unterschiedlichen Ideen konnte eine breite Diffusion erreicht werden, wodurch wiederum viele Teilnehmende der App rekrutiert werden konnten. Von den wenigsten Personen ist allerdings die Intension eines Paradigmenwechsels verstanden worden.

Auch die Entscheidungsträger*innen und die Beteiligten stadtnaher Betriebe nahmen als Ziele des Projektes vor allem wahr, Glück zu operationalisieren und zu messen (G14, G17) und dann herauszufinden, wie sich die Menschen in der Stadt fühlen und was sie negativ oder positiv in ihrer Stadt und ihrem Leben bewerten (G11, G14-16, G18). Glück wurde von den Interviewten der Stadtverwaltung und -politik vor allem in Bezug auf den städtischen Kontext verstanden, also Lebenszufriedenheit im Umfeld der Stadt (G6-9, G11, G14-16, G18-20). Die App könnte ihnen eine längerfristige Datenerhebung zum Zufriedenheitsniveau ermöglichen (G6-8, G10, G15, G17). Ein Interviewter nannte die App „Thermometer der Lebensqualität“ (G14), ein anderer „Spiegelbild der stadtgesellschaftlichen Meinung" (G17). So könne die App niedrigschwellig Stimmungen und Bedürfnisse sowie konkrete Beurteilungen von Projekten abfragen (G9-12, G17) und Themen identifizieren, mit denen sich Stadtentwicklung beschäftigen sollte (DG215).

Dies geschah beispielsweise im Kontext des STEK2030-Prozesses, wo die App als Beteiligungsinstrument genutzt wurde (G6, G9, G11-12, G15, G17, G20). Weitere von den Entscheidungsträger*innen genannte Einsatzmöglichkeit sei die Evaluierung von bereits durchgeführten Projekten, beispielsweise hinsichtlich eines Beitrages zur Verbesserung der Lebensqualität (G9, G15). Aus den 
Daten der App könnten sich dann Handlungsempfehlungen ableiten lassen, die bei zukünftigen städtischen Entscheidungen helfen könnten (G9, G13-14).

Die Entscheidungsträger*innen beschrieben also insbesondere die auf den unteren Ebenen liegenden Ideen hinter "Glücklich in Wuppertal“, die Policies und Programme. Der Gestaltungsanspruch des Paradigmenwechsels wurde von den Entscheidungsträger*innen nicht erwähnt, was vermuten lässt, dass ihnen dies nicht bekannt war. Zwei der Interviewten konnten sich insgesamt nicht mehr genau an die hinter dem Projekt liegenden Ideen erinnern (G11, G19-20).

Die Ideen, die in den Medien sowie von den Entscheidungsträger*innen formuliert wurden, deuten darauf hin, dass zumindest ein Teil der hinter „Glücklich in Wuppertal" stehenden Ideen gut verständlich und einfach kommunizierbar war, was ein Kriterium für erfolgreichen Wandel ist (Campbell 2004, S. 177 f.). Dies trifft jedoch vor allem auf Policies und Programme zu und weniger auf die Paradigmen, die wesentlich seltener kommuniziert und dadurch auch nur sehr begrenzt zu den Entscheidungsträger*innen gedrungen sind.

\subsubsection{Rolle der Wissenschaft}

Wie schon in Abschnitt 5.2.1.2 dargestellt, nahmen die Wissenschaftler*innen unterschiedliche Rollen im Projekt ein: als klassische Forschende und als institutionelle Unternehmer*innen. Das WI nahm die klassische Rolle von Forschenden ein, indem die Wissenschaftler*innen Daten erhoben, neues Wissen über Glück generierten und die Ergebnisse präsentierten (G2, G4-5, DG148, DG152).

Zusätzlich sahen es die Interviewten des WI auch als ihre Aufgabe an, der Stadtpolitik konkrete Vorschläge für Entscheidungen zu unterbreiten und die Idee eines alternativen Wohlstandsparadigmas in der Stadt zu verbreiten (G1-2). Damit gingen sie über die klassische Rolle der neutralen Forschenden hinaus und brachten die Ergebnisse aktiv in die Politik ein. Das WI kann hier daher auch im Sinne eines institutionellen Unternehmers (Campbell 2004, S. 177 f.) und als Theoretiker (Campbell 2004, S. 102) verstanden werden, der seine Ideen von alternativem Wohlstand als Orientierung für die Stadtentwicklung in der Stadt verbreiten will. Hier werden Ansätze einer Modus-3-Wissenschaft deutlich (siehe Abschn. 2.2.2), indem die Forschenden ihre Rolle hinterfragen und institutionelle Rahmenbedingungen ihrer Forschungseinrichtungen bei Bedarf anpassen. Der Projektleiter schrieb der Arbeit des Instituts eine hohe diskursive Kraft zu, durch die Diskurse mitgestaltet und so Einfluss auf die Stadtpolitik genommen werden kann (G1). Den möglichen Einfluss beschrieb er wie folgt:

,[...] eine transformative Wissenschaft heißt, bewusst auch in reale Kontexte zu intervenieren, um besser Veränderungen zu verstehen. Haben natürlich jetzt hier eine 
hervorragende Situation, Diskursintervention zu erproben, indem man selbst zum Aktionsforscher wird. Und das hat uns hier auch sehr gereizt. Diese Diskussion über alternative Wohlstandsverständnisse, die wir insgesamt mit dem anderen Projekt[, WTW,] angestoßen haben, hier jetzt über so eine „Glücklich in Wuppertal“-App nochmal intervenieren konnten." (G1)

Das WI verfügt über zahlreiche Netzwerke und Kontakte zur lokalen Zivilgesellschaft sowie zu einigen Akteur*innen aus der Stadtpolitik und -verwaltung, die es mit in das Projekt einbrachte und weiter ausbaute. Insbesondere der Präsident des Instituts verfügt über ein enges Netzwerk zu anderen Organisationen und ist hoch angesehen. Er selbst war im Projekt als Leiter auch aktiv in die Ansprache von Kontakten involviert, beispielsweise zu den lokalen Unternehmenspartner*innen und zum Oberbürgermeister (G1, G4-5). Seine Rolle im Projekt, das Verbreiten der Ideen des Projektes innerhalb seines Netzwerkes, wurde als sehr hilfreich für das Projekt wahrgenommen (G5, G9).

Das WI ist damit auch als epistemische Gemeinschaft (Campbell 2004, S. 104 f.; Haas 1992, S. 27-29) zu verstehen, die versucht die Politik mit seinen Ideen zu beeinflussen. Laut Haas (1992, S. 27-29) müssen epistemische Gemeinschaften in ihren Kontexten gut etabliert und anerkannt sein, damit sie erfolgreich Politik beeinflussen können. Dies trifft auf das WI zu (G1-2, G14, G17).

Die anderen, nichtwissenschaftlichen Projektpartner*innen fanden die vom WI eingenommene Rolle gut und richtig und befürworten es, wenn die Wissenschaftler*innen ihre Forschungsergebnisse und Ideen in reale Kontexte integrieren und voranbringen. Sie wünschten sich also diese Rolle der Theoretiker*innen oder epistemischen Gemeinschaften von den Wissenschaftler*innen. Von ihnen wurden die beteiligten Wissenschaftler*innen mit Respekt behandelt und als „Fachleute“ beschrieben, die Wissen einbringen und verbreiten (G3, G5).

Auch Interviewte außerhalb des Projektes sprachen sich für die lokale anwendungsorientierte Forschung aus (G6-9, G11, G13, G16) und nahmen die Arbeit der beteiligten Wissenschaftler*innen und die Projektpräsentationen als glaubwürdig, wissenschaftlich korrekt und gleichzeitig transparent und nachvollziehbar wahr (G8, G15-17) und beschrieben die Wissenschaftler*innen als Berater*innen (G5-6, G14). Sie wurden dabei als offen wahrgenommen, hätten das Gespräch gesucht und Vernetzung betrieben (G8-9, G13). Auf die Frage, wie er die Wissenschaftler*innen wahrgenommen habe, beschrieb ein Mitarbeiter der Stadtverwaltung:

„Also ich würde sagen, ja als Partner und Berater. Vielleicht Berater am ehesten, der bemüht ist, die Erkenntnisse, die er gewonnen hat, auch weiterzugeben, damit sie 
nicht nur irgendwie Erkenntnis bleiben, sondern auch im Konkreten dann weiterhelfen können."(G6)

Fast alle Interviewten waren der Meinung, dass Wissenschaft dabei auch normativ argumentieren darf und versuchen sollte, gesellschaftlich etwas zu bewegen und Handlungsempfehlungen auszusprechen (G7, G9, G11, G13, G18, G20). Dies sei besonders wertvoll, da die Wissenschaft als Impulsgeberin Ideen und Denkanstöße liefern könne, auf die die Stadtverwaltung selbst nicht käme (G11, G13-14, G16). Im STEK2030-Prozess und bei W2025 sei dies sehr positiv gewesen (G15, G17). Die wissenschaftlichen Impulse würden außerdem helfen, von emotionalen Diskussionen wegzukommen und bisher eher intuitive Argumente wissenschaftlich $\mathrm{zu}$ fundieren (G17-18). Ein Interviewter meinte, die Wissenschaft könne auch konkrete Forderungen stellen, wie mit ihren Ergebnissen und Vorschlägen umgegangen werden solle (G15).

Das WI kann also als epistemische Gemeinschaft im Sinne von Haas (1992, S. 27-29) und Campbell (2004, S. 104-105) verstanden werden (siehe Abschn. 3.2.2), die auch über die notwendigen Netzwerke verfügt, im städtischen Kontext gut angesehen ist und als Berater*in angefragt wird. Gleichzeitig agierte das WI auch als institutioneller Unternehmer, der nicht nur berät, sondern selbst Ideen aktiv voranbringt und Zugang zu Entscheidungsträger*innen sucht.

\subsubsection{Rahmenbedingungen}

Für den Erfolg neuer Ideen im Diskurs und das Anstoßen von Wandel spielen die Rahmenbedingungen eine große Rolle: Sowohl der institutionelle Kontext der Stadt als auch weitere Veränderungen und wahrgenommene Probleme, für die die Ideen möglicherweise eine Lösung darstellen können. Dieser Hintergrund wird daher im Folgenden anhand der von den Interviewten und in den Dokumenten im Zusammenhang mit „Glücklich in Wuppertal“" genannten Themen beschrieben ${ }^{5}$. Daraufhin (Abschn. 5.2.3) wird erläutert, wo die Ideen erfolgreich anknüpfen konnten und wo nicht.

\footnotetext{
${ }^{5}$ Dadurch, dass es sich bei den beiden untersuchten Fällen um denselben städtischen Kontext handelt, kommt es hier zu einigen Überschneidungen mit Abschnitt 5.1.2, den Rahmenbedingungen des ersten Falles. Da es hier allerdings darum geht, welche Rahmenbedingungen wie Krisen oder institutionelle Veränderungen - von den Interviewten wahrgenommen und im Zusammenhang mit diesem zweiten Fall genannt werden, wird eine separate Ausführung aus methodischen Gründen als notwendig angesehen.
} 
In den Interviews und Dokumenten wurde das Projekt in einen spezifischen institutionellen Kontext eingebettet und mit verschiedenen Herausforderungen in Wuppertal in Verbindung gebracht, von denen einige als für den Erfolg des Projektes förderlich, andere als erschwerend empfunden wurden. Die Koalition aus SPD und CDU, die in Wuppertal im Stadtrat seit Jahren bestand, wurde als einschränkend wahrgenommen, weil der Oberbürgermeister in dieser Konstellation oft nicht über eine Mehrheit für seine Ideen verfügt (G1). Im Laufe des Interviewzeitraumes wurde diese Koalition aufgelöst und die dadurch neu entstandene Situation als offener für Veränderungen beschrieben (G15), was jedoch auf einen Zeitpunkt nach Abschluss des Projektes fällt.

Als prägend für städtische Entwicklungen in Wuppertal wurde außerdem die seit Jahrzehnten andauernde schlechte wirtschaftliche Lage genannt und die hohen Schulden der Stadt, wodurch Wuppertal über wenig Entscheidungsspielraum bei städtischen Entscheidungen verfügt (G1-2, G4, G7, G10, G15, G17, G19-20, DG6, DG23, DG104). Über lange Zeit wurde Personal in der Verwaltung eingespart, so dass nun viele Ideen nicht umsetzbar sind (G15). So gab es zum Beispiel in der Vergangenheit städtische Befragungen zur subjektiven Wahrnehmung der Bevölkerung, die aber aufgrund von Personalwechsel und -einsparungen nicht weitergeführt wurden (G6).

Dieses stellt das mit Abstand am häufigsten in den Datenquellen genannte Problem dar und die Interviewten waren der Meinung, dass es auch in der Stadtgesellschaft und -politik als Problem angesehen und so kommuniziert wird (G1-2, G8, G13, G15, G19-20). Da diese Herausforderung von zahlreichen Entscheidungsträger*innen wahrgenommen wird und in der Stadtpolitik Lösungen debattiert werden, kann sie durchaus als Krise, wie sie im diskursiven Institutionalismus als Voraussetzung für Veränderung genannt wird (siehe Abschn. 3.3.2), verstanden werden. Dies bestätigt auch den in Abschnitt 2.4 genannten Fokus von Stadtpolitik und -verwaltung auf wirtschaftliche Aspekte.

Weitere Probleme die genannt wurden, sind eine Segregation und Polarisierung zwischen verschiedenen Bevölkerungsgruppen und Stadtteilen (G1-2, G11, DG32, DG164, DG215), der demografische Wandel (G20, DG213, DG215), die hohe Armutsrate, die private Überschuldung und Arbeitslosigkeit (G8, G11, G13, DG32, DG164, DG213) sowie niedrige Bildungsabschlüsse in vielen Bevölkerungsgruppen (DG164). Auch diese Probleme können als Krisen verstanden werden, die die Stadtpolitik zu lösen versucht.

Damit zusammen hängen das schlechte Image der Stadt und das meist schlechte Abschneiden Wuppertals in Städterankings (G2, DG1, DG164). Auch das Selbstbild der Stadt ist eher negativ und positive Aspekte geraten oft in den Hintergrund (G12, G18). Einige Probleme sind direkt im Stadtbild sichtbar, wie 
die hohe Leerstandsquote und die unbelebten Innenstadtbereiche, die neue Konzepte in Zeiten des Online-Handels erfordern würden (G2, G18-19) sowie die teilweise mangelnde Sauberkeit und schlechte Aufenthaltsqualität (G14, DG213). Mittlerweile wächst die Einwohnerzahl Wuppertals wieder, so dass zumindest der Wohnungsleerstand verringert werden konnte. Jedoch ist die Stadt nun damit konfrontiert, sich von einer schrumpfenden zu einer wachsenden Stadt zu entwickeln und so städtische Entscheidungen zu überdenken (G12, DG104). Herausforderung ist nun, geeigneten Wohnraum zu schaffen (G14-16, G18-19) ohne viel Fläche dafür zu versiegeln (G15, G19) sowie neue Angebote an Kindergärten und Schulen bereitzustellen beziehungsweise zu verbessern (G18-19). Damit zusammen hänge auch die andauernde Zuwanderung aus dem Ausland nach Wuppertal, die sowohl in Bezug auf Wohnraum als auch auf die Integration Herausforderungen mit sich bringe (G10, G12-13, G18, G20, DG213). Auch diese genannten Themen wurden als Krisen verstanden, die potenzielle Anknüpfungspunkte für neue Ideen darstellen könnten und von den Interviewten im Zusammenhang mit „Glücklich in Wuppertal“ genannt wurden. Ob hier eine Anknüpfung gelang, wird später ebenfalls analysiert.

Zusätzlich zu diesen Herausforderungen werden aktuelle Streitpunkte im städtischen Diskurs wahrgenommen, insbesondere wurde hier die Diskussion um Großprojekte genannt, wie der Umbau des Bahnhofsvorplatzes Döppersberg, die Planung einer Seilbahn oder des Pina-Bausch-Tanzzentrums (G1-3, G6-8, G11, G16-17, G19, DG164). Es sei schwer zu vermitteln, dass einerseits in Großprojekte investiert wird, die Fördergelder jedoch zweckgebunden seien und nicht an anderen Stellen ausgegeben werden könnten (G7, G17). Außerdem seien bei diesen Projekten die Gegenstimmen meist lauter als die der Befürwortenden, was zu einem falschen Bild der Meinung der Wuppertaler*innen führe (G17). Diese Diskussionen um die Großprojekte wurden als zentrale, offen diskutierte Themen in der Stadt beschrieben (G8, G17, G19, DG164) und können in diesem Sinne ebenfalls als Krisen verstanden werden, da sie neue Formen der Beteiligung und Kommunikation der Politik erfordern. Hier könnte "Glücklich in Wuppertal“ womöglich eine Lösung bieten.

In diesem Zusammenhang wurde von einigen der Interviewten sowie in Dokumenten eine Krise der repräsentativen Demokratie genannt, in der viele Menschen bei klassischen Beteiligungsverfahren in der Stadt nicht mehr erreicht werden. Viele würden sich gar nicht mehr oder stattdessen nur auf kleinräumiger Ebene, beispielsweise in den Quartieren, einbringen wollen (G5, G7, G11, DG210). Ein Interviewter schloss aus dem veränderten Umgang vieler Einwohner*innen ihm als Stadtverwaltungsvertreter gegenüber auf eine Vertrauenskrise in das politische System auch auf kommunaler Ebene. Viele würden der Kommunalverwaltung 
und -politik gegenüber weniger respektvoll begegnen (G20). Zwei Interviewte beschrieben Kritik von Seiten der Bevölkerung gegenüber der Verwaltung, da ihre hohen Erwartungen nicht erfüllt würden (G9, G20).

Bezogen auf Bürgerbeteiligung in Wuppertal fiel die Zeit des Projektstartes mit der Gründung eines Beteiligungsdezernats in der Stadtverwaltung zusammen kurz darauf folgte die Einrichtung eines Beirates für Bürgerbeteiligung (G7-8) und damit in die Zeit einer Institutionenentwicklung. Personalwechsel bei den Mitarbeitenden, die Abwahl des gerade erst berufenen Dezernenten und Umwandlung des Dezernats in eine Stabsstelle Bürgerbeteiligung und Engagement (siehe Abschn. 2.4) brachten jedoch wieder Einschränkungen mit sich, unter anderem da nun weniger Handlungsspielraum besteht als zu Beginn nach der Schaffung des neuen Dezernates (G7). Trotzdem ist insgesamt eine Stärkung des Bereichs Bürgerbeteiligung in der Stadt durch die zusätzlichen personellen Ressourcen zu beobachten (G8).

„Es gab halt so einen Aufbruch sage ich mal, oder hoffe ich mal, aber man ist halt diesen Schritt gegangen: Man hat ein Dezernat eingerichtet, man hat gesagt, wir wollen uns mehr in diese Richtung entwickeln. Und in dem Zuge, oder in dem Sinne ist ja vielleicht auch eine Glücksapp zu sehen, als eine Idee, sich auch zu öffnen. Und dann war einfach so ein... so ein Cut da drin.“ (G7)

Als weitere Prozesse während des Untersuchungszeitraumes, die für die Verbreitung der Ideen von „Glücklich in Wuppertal“ relevant sein könnten, wurde der Prozess der Entwicklung eines neuen Stadtentwicklungskonzeptes (STEK2030) genannt, der an eine externe Agentur vergeben wurde, die dann Kontakt zu den Beteiligten von „Glücklich in Wuppertal“ aufgenommen und die App in den Prozess integriert hat (G4, G6, DG189). Parallel lief noch das vorhergehende Stadtentwicklungskonzept W2025, bestehend aus einer Reihe von Projekten, das zu der Zeit auf der Suche nach geeigneten Evaluationskriterien für die Projekte war (G4, G9, G15, G18, G20, DG213). Im Laufe des Untersuchungszeitraumes kam dieser Prozess dann jedoch ins Stocken, so dass keine weiteren Treffen stattfanden (G17).

Neben diesen größtenteils lokalen Veränderungen und Krisen wurden auch Herausforderungen genannt, die über Wuppertal hinaus eine Rolle spielen und vom nationalen Diskurs beeinflusst sind. So wurden in Dokumenten Umweltprobleme und der Klimawandel als Krisen genannt (DG6, DG164, DG204, DG215, DG23). Die projektinternen Forschenden des WI schlussfolgerten daraus die Notwendigkeit einer Nachhaltigkeitstransformation (DG6, DG164), und auch einige 
andere Interviewte sowie Dokumente beschrieben die Notwendigkeit, Klimaschutz und Klimafolgenanpassung zu betreiben (G14-16, DG215). Weiterhin wurde die Notwendigkeit einer Verkehrswende insbesondere zur Verringerung der Feinstaubbelastung in der Stadt genannt (G13-16, G18-19). Die Feinstaubbelastung wurde auch als offen kommuniziertes Problem beschrieben und ist vor allem durch drohende Dieselfahrverbote und deutschlandweite Diskussionen darüber in den Fokus geraten (G13-14, G16, G18-19).

Ein Widerspruch, der von einigen Interviewten wahrgenommen wurde, ist zwischen der Verwendung wirtschaftlicher Kennzahlen wie dem BIP als Indikator für Lebensqualität und der Differenz zur vielschichtigen Bedeutung von Lebensqualität. Alternative Indikatoren werden oft nicht betrachtet und der Blick lediglich auf wirtschaftliche Aspekte gerichtet. Gleichzeitig fehlen aber auch oft subjektive Daten, um andere Indikatorensets wirklich nutzen zu können (G13, DG2-4, DG13, DG23, DG32, DG93, DG204, DG210). Dies wurde teilweise auch in der Presse so kommuniziert (DG13, DG32).

Ein Interviewter nannte außerdem einen Widerspruch zwischen verschiedenen Entwicklungspfaden der Stadt: Einerseits einem investorengetriebenen Handeln und Bestreben nach Wirtschaftswachstum und andererseits alternativen Projekten aus der Zivilgesellschaft, welche sich die Freiräume und Leerstände Wuppertals zunutze machen und mit denen sich die Stadt gerne nach außen präsentiert (G2). Aus den Dokumenten und Interviews ist aber nicht erkennbar, dass dieser Widerspruch offen im städtischen Diskurs wahrgenommen und kommuniziert wird.

Weiterhin wurden verschiedene Herausforderungen nur in einzelnen Interviews und Dokumenten genannt, so der Einfluss großer Unternehmen auf die Stadt durch den Abzug von Arbeitsplätzen (G10), die Schließung kleiner Polizeiwachen und anderer Orte lokaler Quartierskommunikation (G19), das Erstarken von Populismus und rechten Strömungen (G8) und das Thema Digitalisierung (DG213). So war eine weitere Rahmenbedingung, in der das Projekt agierte und versuchte daran anzuknüpfen, die Auswahl Wuppertals als eine von fünf digitalen Modellkommunen in NRW, was neue Finanzierungsmöglichkeiten von Projekten im Bereich Digitalisierung mit sich brachte (DG204).

Zusammenfassend lässt sich also sagen, dass das Projekt in eine Zeit institutioneller Veränderungen fiel, von denen die Stärkung des Bereichs Bürgerbeteiligung in der Stadt sowie die Ablösung eines Stadtentwicklungskonzeptes durch ein anderes als besonders relevant für den untersuchten Fall wahrgenommen wurden. Die Schwierigkeit, die Bürgerschaft bei stadtpolitischen Themen zu erreichen und die Konflikte über Großprojekte, werden sowohl öffentlich in der Presse als auch von den Entscheidungsträger*innen und in städtischen Dokumenten 
als Herausforderung kommuniziert und können als Krisen verstanden werden. Zusätzlich wurden die hohen Schulden der Stadt als Krise beschrieben, die auch als solche von fast allen Entscheidungsträger*innen kommuniziert wurde. Insbesondere scheint diese Krise andere Krisenwahrnehmungen mit sich zu bringen, wie den geringen Handlungsspielraum der Kommune, das schlechte Image der Stadt und die hohe Armutsquote der Bevölkerung. Andere Widersprüche und Herausforderungen wie Umwelt- und Klimaschutz oder die Kritik am Fokus auf wirtschaftliche Entwicklungen wurden hauptsächlich von den Wissenschaftler*innen und einzelnen anderen Personen wahrgenommen und von den Entscheidungsträger*innen weniger oder gar nicht mit „Glücklich in Wuppertal“ assoziiert. Inwieweit „Glücklich in Wuppertal“ an diese Krisen und institutionellen Veränderungen anknüpfen konnte und was sich als förderlich und was als hinderlich herausstellte, wird im folgenden Abschnitt dargestellt.

\title{
5.2.3 Anknüpfungspunkte und Umsetzbarkeit der Ideen
}

In Bezug auf die von einigen Interviewten wahrgenommene Krise der repräsentativen Demokratie und die Schwierigkeiten, die Bürgerschaft in Beteiligungsprozessen zu erreichen, sahen einige Entscheidungsträger*innen die App als eine mögliche Lösung, um zusätzliche Personen in Beteiligungsprozesse hereinzuholen (G1-3, G5, G7, DG210). So könnten parteienübergreifend und niedrigschwellig kontroverse Themen abgefragt werden, beispielsweise zu geplanten Großprojekten (G11, G16).

\begin{abstract}
„Also, wir wissen ja aus allen unseren Beteiligungsverfahren, dass aktiv vor allem eben die sind, die dagegen sind. Und, dass es echt schwer ist, egal ob man in einer Veranstaltung oder sonst wo sitzt, die zu motivieren, die was gut finden. Und ich glaube, jetzt liegt in so einem Instrument erstens das Risiko, dass es da genauso ist. Andererseits aber eben auch die Chance, dass es anders sein könnte, weil ich eben nicht in einer Veranstaltung aufstehen muss und sagen muss, hey, ich finde es aber gut, auch wenn ich jetzt ganz alleine bin, oder so.“(G11)
\end{abstract}

Auch insgesamt wurde die App als relevant für Stadtentwicklung gesehen, weil der Bereich Stadtentwicklung sich ebenfalls damit beschäftigt, die Lebensqualität zu verbessern (G14, G16, G19-20). Außerdem wurde sie als geeignet für die Quartiers- und Bezirksebene gesehen (G9, G11-12). Ebenso könnte sie als Mängelmelder genutzt werden (G10, DG210) und sei besonders anschlussfähig an die Digitalisierungsbestrebungen der Stadt (G10, G12). Als Schwierigkeit wurde 
jedoch gesehen, dass die Stadtverwaltung schon über viele Daten verfügt und Probleme hat, damit umzugehen (G17-18).

Von einigen Nutzer*innen wurde jedoch auch angemerkt, dass das Ausfüllen der Fragen sehr lange dauert, Durchhaltevermögen erfordert, da oft im Laufe des Tages immer wieder Fragen aufgetaucht und diese teilweise unlogisch aufgebaut seien, weshalb das Erreichen von Personen aller Bevölkerungsgruppen möglicherweise schwierig sein könnte (G17, DG51, DG59).

Die bisher im Projekt entstandenen Daten waren noch nicht über einen langen Zeitraum erhoben und nicht repräsentativ, so dass daraus noch keine aussagekräftigen Schlussfolgerungen für städtische Entscheidungen abgeleitet werden können (G1, G3, G14-15). Bisher konnten die meisten Entscheidungsträger*innen keinen konkreten Nutzen für ihre Arbeitskontexte erkennen (G8, G12, G15, G20). Eine bessere Aufbereitung der Ergebnisse hätte vermutlich insbesondere der Stadtpolitik geholfen (G16, G18-19). Die Ideen von „Glücklich in Wuppertal“ stellten sich als dort anschlussfähiger heraus, wo es um konkrete Fragen der Stadtpolitik ging, beispielsweise den Bau einer Seilbahn (G1-2, G4-5). Allgemeinere Aussagen und Ideen über Glück und Lebensqualität und neue Leitbilder scheinen dagegen weniger anschlussfähig und schwieriger nutzbar zu sein, sowohl für die Stadt als auch für die Pressekommunikation (G1-2, G4, G16, G18). Auf der höheren Ebene von Ideen, den Paradigmen, war die Anschlussfähigkeit also weniger vorhanden.

Konkret anschlussfähig waren die Ideen an die Entwicklung der Stadtentwicklungskonzepte STEK2030 und W2025 (G4, DG189, DG213), hier fiel der Projektzeitraum in einen günstigen Moment institutionellen Wandels. Bei W2025 wurde nach einem konkreten Maßstab zur Evaluierung der laufenden Projekte gesucht, wo die App gut anknüpfen konnte (G9, G15, G18, G20, DG80). Der Vertreter des Stadtmarketings beschrieb die Verknüpfung wie folgt:

\footnotetext{
„Also in der 2025, also in dem Strategiekreis war ja auch die Herausforderung, sie haben 13 Schlüsselprojekte, die als Ziel haben, ja, auf die Dauer der Stadt eine neue Lebensqualität, oder eine andere zu geben. Und dann ist ja immer genau die Frage, wie ist denn Lebensqualität messbar. Wir können über Wirtschaftsrankings, alles Mögliche, kannst du alles messen. Aber ein Gefühl zu messen ist extremst schwierig. Und dann habe ich das auch unterstützt, dass das da auch vorgestellt wird.“(G17)
}

Die Art der Treffen des W2025-Kreises stellten sich aber als ungeeignet für einen tieferen Austausch heraus (G15). Auch beim STEK2030-Prozess bekam die App eine Relevanz und wurde genutzt, um andere Zielgruppen zu erreichen als die Präsenzveranstaltungen und mehr Informationen über die Einstellungen der Bevölkerung zu ermitteln (G9, G11, G14, G16). An die Stadtentwicklungsprozesse und die Krise der städtischen Beteiligungsinstrumente konnte „Glücklich 
in Wuppertal" mit seinem Angebot also insgesamt gut anknüpfen. Auffällig ist hier, dass die Anknüpfung an beide Prozesse sich im Laufe des Untersuchungszeitraumes ergab und nicht von Anfang an geplant war. Hier wurde auf passende Prozesse reagiert und eingegriffen. Erschwerend kam dann aber die weitere Entwicklung von W2025 (siehe Abschn. 5.2.2) und die teils etwas zu langwierige Nutzung der App hinzu, was jedoch einer ersten Nutzung des Instruments bei STEK2030 nicht im Wege stand.

Auf diese Ebene bezogen - die Nutzung eines Befragungsinstruments zur Erhebung subjektiver Daten - würden durch die Umsetzung der Idee keine tiefgreifenden Veränderungen vorausgesetzt. Die vorherrschenden Paradigmen könnten erhalten bleiben und es würde lediglich ein neues Instrument eingeführt, was gegebenenfalls zu anderen kleinteiligen Entscheidungen führen würde, also einem Wandel erster Ordnung (G2-6, G8-10, G16-17). Eine grundlegende Kritik an den Entscheidungen wäre aber nicht zu erwarten (G6, G8-10, G16-17).

Dieses Angebot der App als neues Beteiligungsinstrument und zur Lösung der Herausforderungen durch sowohl geringe finanzielle Mittel für Bürgerbeteiligung als auch niedrige Beteiligungsquoten, bezieht sich auf die Ideen von „Glücklich in Wuppertal" auf der ersten und teilweise zweiten Ebene. Auf der Ebene von Paradigmen schließt „Glücklich in Wuppertal“ an die Debatte und Forschung über alternativen Wohlstand und Definitionen von Lebensqualität an. Wohlstand wird dabei nicht nur unter ökonomischen Gesichtspunkten verstanden, sondern bezieht andere Aspekte ein, die die Menschen in der Stadt glücklich machen. So könnte das Projekt Daten zur subjektiven Lebenszufriedenheit und deren Einflussfaktoren liefern, die bisher fehlen, um Lebensqualität umfassend zu messen. Das Projekt könnte so dem BLI-u zur Etablierung verhelfen, indem fehlende Daten dafür erhoben werden (G13, DG2-4, DG13, DG23, DG32, DG93, DG204).

Auf der Ebene der Paradigmen ist die Anschlussfähigkeit und Umsetzbarkeit aber als schwieriger anzusehen und eine Orientierung politischer Entscheidungen an alternativem Wohlstand würde tiefgreifende Veränderungen dritter Ordnung voraussetzen. Auf die alternativen Paradigmen bezogen waren die Ideen des Projektes eher an nachhaltigkeitsorientierte Projekte und Akteur*innen in Wuppertal anschlussfähig, die bereits ähnliche Ziele und alternative Entwicklungspfade verfolgten (G2). Die Stadt Wuppertal mit ihren hohen Schulden orientiert sich weiterhin hauptsächlich an Zielen des Wirtschaftswachstums, so dass die Idee eines neuen Wohlstandsmaßes und einer Abwendung von rein materiellen Aspekten weniger anschlussfähig war und dieses Problem sich eher als hinderlich herausstellte. Hier war also zwar eine Krise vorhanden und wahrgenommen, doch die Ideen konnten nicht daran anknüpfen, da entweder der Handlungsdruck 
nicht hoch genug schien oder die Entscheidungsträger*innen andere Lösungen als geeigneter ansahen.

Erleichtert wurde die Kommunikation jedoch durch den niedrigschwelligen Begriff Glück. Das Konzept Glück wurde als geeigneter und verständlicher Begriff gesehen (G2, G4, DG204). Glück abzufragen sei erst einmal ungewöhnlich aber dadurch interessant und innovativ (G14, G20), der Fokus auf das Positive gut (G2, G11, G18).

„Nach dem Motto hey, wir machen hier was für Wuppertal, in Wuppertal kann man auch glücklich sein. Hey, Wuppertal ist doch gar nicht so schlecht wie immer alle sagen." (G2)

So könnte die App zu einer Verbesserung des Wuppertaler Images beitragen, einer Stadt deren Bewohner*innen vorgeworfen wird, sich häufig zu beschweren und vor allem auf das Negative zu schauen (G8, G13, G17-18). Dadurch ergibt sich auch eine Anschlussfähigkeit an das Wuppertaler Stadtmarketing (G17, G20). Eine Interviewte beschrieb den Vorteil des Glücksbegriffs:

\begin{abstract}
„Diese Glückssache läuft auf einer allgemeineren Ebene. Das versteht ein Kind, [...] das verstehen ältere Menschen, wo ich glaube, das ist handhabbarer. Weil es einmal... es erzeugt so ein Lächeln und es wirkt nicht so nach dem Motto, da muss ich wer weiß wie, da muss ich zehn Bücher lesen, bevor ich das verstehe. Sondern zu sagen, diese positive Wahrnehmung, und das ist was Universelles. Da gibt es keine Sprachbarrieren, und deswegen finde ich das ein schönes Instrument, weil das eben weg ist von diesem akademischen Level einfach. Auch wenn es akademisch fundiert ist, aber es ist einfach greifbarer.“(G18)
\end{abstract}

Der Glücksbegriff wurde aber auch kritisch gesehen, da Glück tagesformabhängig und nicht unbedingt Ziel von Politik sei (G8, G12). Auch ein Projektbeteiligter merkte an, dass wenn Politik danach ausgerichtet würde, was das Glück der Bevölkerung maximiert, dies nicht unbedingt mit den Zielen der Nachhaltigkeitstransformation zusammenpassen würde, sondern diesen sogar teilweise widerspreche (G2).

Zusammenfassend lässt sich also festhalten, dass „Glücklich in Wuppertal“ durch den Begriff Glück gut ankam, an die laufenden Stadtentwicklungsprozesse gut anschließen konnte und sich diese als geeignete Gelegenheitsfenster herausstellten. So konnte auch an die Schwierigkeit, Menschen für Bürgerbeteiligung zu erreichen, angeknüpft und die App als ein Lösungsvorschlag präsentiert werden. Daneben konnte an die finanzielle Krise der Stadt nur insofern angeknüpft werden, als positive Aspekte in den Vordergrund gerückt wurden und 
das Beteiligungsinstrument gerne genutzt wurde, um Ausgaben für städtische Beteiligungsinstrumente einzusparen. Grundlegender gelang es jedoch nicht, den Fokus weg von finanziellen Aspekten, hin zu anderen Aspekten von Lebensqualität zu lenken. Im Gegenteil erschien der mangelnde Entscheidungsspielraum der Verwaltung sogar als hinderlich, weil so neue Ideen alleine schon aus Kapazitätsgründen nicht aufgegriffen wurden. Projektimmanenter hinderlicher Faktor schien außerdem auch in der konkreten Ausgestaltung der App zu liegen, die laut einigen Nutzer*innen aufwändig sei und teilweise unlogische Fragekombinationen enthalte.

\subsubsection{Beobachtbare Veränderungen}

Nachdem beschrieben wurde, wo „Glücklich in Wuppertal“ anschließen konnte und wie groß die vorausgesagten Veränderungen waren, wird nun dargestellt, wo es bereits zu Veränderungen kam, wo Ideen bereits umgesetzt oder gar Politikveränderungen geschehen sind. Im Laufe des Untersuchungszeitraumes konnten schon kleinere Prozesse beobachtet werden, bei denen durch „Glücklich in Wuppertal" neue Ideen verbreitet oder sogar umgesetzt werden konnten. Die App wurde beim STEK2030-Prozess erstmals als Beteiligungsinstrument verwendet. Dazu wurden im Vorfeld einer Zukunftswerkstatt Fragen eingespielt und die Antworten ausgewertet. In der Zukunftswerkstatt konnten diese dann angesehen und analog ergänzt werden (G2, G4, G6, G13-14, DG204, DG208, DG210, DG215, DG188, DG190, DG207). Wie die Ideen der institutionellen Unternehmer*innen in die Ergebnisse des STEK2030 eingeflossen sind, ist zum Zeitpunkt der Untersuchung jedoch noch nicht erkennbar (G13, G20). Ein Interviewter meinte, sie seien als eine von mehreren Quellen eingeflossen (G14). Beim W2025-Prozess entschied man sich für eine Nutzung der App und diese wurde auch im Sachstandsbericht über die Stadtentwicklungsprojekte erwähnt (DG213). Bisher ist es allerdings nicht zu einer Nutzung der App als Evaluierungsinstrument gekommen und auch keine konkrete Umsetzung geplant (G9). Ein Interviewter meinte jedoch, dass die Ideen von „Glücklich in Wuppertal“ schon jetzt neue Denkanstöße im Hinblick auf die Projekte von W2025 und den STEK2030-Prozess geliefert hätten (G16).

Durch die starke Öffentlichkeitsarbeit von WI und Sparkasse sowie die Berichte in der WZ ist außerdem ein Diskurs über Glück in der Stadt zu beobachten. Einige Expert*innen nehmen an, dass durch die zahlreichen Presseartikel Leser*innen dazu angeregt wurden, über Glück und Zufriedenheit nachzudenken (G1-2, G4). Die Sparkasse hat in ihrem Kund*innenmagazin die Begriffe 
Wohlstand und gutes Leben vermehrt genutzt und die gemeinsamen Ideen in ihre eigenen Aktivitäten integriert. Hier hat also ein leichter Wandel in den Diskursen des Unternehmens stattgefunden (G3).

Weiterhin waren erste Erhebungen erfolgreich und es konnten erste Erkenntnisse gewonnen werden, beispielsweise über die Zufriedenheit der Bevölkerung unterschiedlicher Stadtteile im Vergleich oder die Zufriedenheit mit bestimmten Aspekten der Stadt, die sowohl bei Veranstaltungen präsentiert, als auch auf der Projekthomepage publiziert wurden (G2-3, G6-7, G9, G13). Ein Panel von 1000 Personen zu etablieren, ist allerdings noch nicht gelungen und die bereits erhobenen Daten sind nicht repräsentativ (G1-3, G6, G9, G13).

Insgesamt ist also eine breite Diffusion der Ideen der unteren beiden Ebenen, insbesondere der Policies, teilweise auch neuer Programme in die Bürgerschaft und Stadtverwaltung hinein zu beobachten, vereinzelt auch in Richtung Unternehmerschaft und Stadtpolitik. Auf diesen Ebenen erster und zweiter Ordnung gab es auch bereits kleine Veränderungen. Auf der höheren Ebene von Paradigmen kam es bisher zu einer wesentlich geringeren Diffusion und zu einem Wandel dritter Ordnung ist es bislang nicht gekommen.

\subsubsection{Zusammenfassung und Abgleich mit den Prognosen}

Im folgenden Abschnitt werden die in den vorherigen Abschnitten dargelegten Analyseergebnisse zusammengefasst und die Prognosen der Kongruenzanalyse (siehe Abschn. 4.3) mit den Beobachtungen abgeglichen, um herauszufinden, ob die Theorie des diskursiven Institutionalismus die Entwicklungen im Fall von „Glücklich in Wuppertal“" erklären kann.

Bezogen auf das Vorhandensein, die Wahrnehmung und die Kommunikation einer Krise zeigte die Analyse, dass insbesondere die hohen Schulden der Stadt als solche verstanden werden können. Die Ideen von „Glücklich in Wuppertal“ konnten hier zwar nicht als Lösung der eigentlichen Krise angeboten werden, jedoch gut anknüpfen, da die mit dem schlechten Image kämpfende Stadt gerne positive Bilder in den Fokus rückt. Den Blick von den ökonomischen Aspekten hin zu anderen Zielen zu lenken und stadtpolitische Entscheidungen daran auszurichten - eine Idee der institutionellen Unternehmer*innen - wurde jedoch bisher nicht erreicht. Außerdem wurde es als schwierig beschrieben, die Bevölkerung bei stadtpolitischen Fragen zu erreichen, ebenfalls eine von Entscheidungsträger*innen öffentlich kommunizierte Krise. Die Annahme, dass Krisen in der Stadt wahrgenommen und kommuniziert wurden, konnte also bestätigt werden, 
womit eine der notwendigen Voraussetzungen für Politikwandel vorliegt (siehe Abschn. 3.3.2).

In Bezug auf Bürgerbeteiligung fiel das Projekt „Glücklich in Wuppertal“ in einen günstigen Zeitraum, wo dieses Thema gerade im Fokus stand und durch eine neue Stabsstelle und zusätzliches Personal gestärkt wurde. Hier knüpft ein Teil der Ideen der institutionellen Unternehmer*innen an: Die App als neues Beteiligungsinstrument zu nutzen und Politik so mehr an der Meinung der Bürgerschaft auszurichten. Diese Idee würde veränderte Policies mit sich bringen und eine Veränderung erster Ordnung bedeuten (Hall 1993, S. 278, siehe Abschn. 3.3.1). Hier können bereits erste Erfolge im Zusammenhang mit STEK2030 und W2025 beobachtet werden. Gleichzeitig bezweckten die Projektbeteiligten eine Verschiebung des stadtpolitischen Fokus von wirtschaftlichen Aspekten auf Lebensqualität und eine Stärkung der Wohlstandsindikatoren, also ein neues Paradigma, das Entscheidungen leitet. Eine Umsetzung hier würde einen Wandel dritter Ordnung mit sich bringen.

Diese Ideen unterschiedlicher Ebenen wurden von den institutionellen Unternehmer*innen des Projektes vorangebracht und haben mit der Unterstützung von Medien und Zivilgesellschaft eine breite Diffusion erfahren. Dabei zeigte sich eine einfachere Kommunikation und höhere Anschlussfähigkeit der auf Beteiligung und Glücksmessung bezogenen Ideen als des angestrebten Paradigmenwechsels.

Die Annahmen, dass institutionelle Unternehmer*innen vorhanden seien, die neue Ideen voranbringen, können also auch bestätigt werden. Damit sind weitere Voraussetzungen für Politikwandel erfüllt. Allerdings verfolgten nicht alle Projektpartner*innen alle Ideen in gleichem Maße, so dass vor allem die auf den unteren beiden Ebenen liegenden Ideen kommuniziert wurden und weiter diffundiert sind. Die Diffusion fand sowohl breit in die Bürgerschaft hinein statt, als auch gezielt zu Entscheidungsträger*innen. Anwendungen und damit Veränderungen erster Ordnung konnten bereits beobachtet werden. Veränderungen höherer Ordnung sind bisher nicht eingetreten.

Insgesamt konnten also die vom diskursiven Institutionalismus prognostizierten Veränderungen erster Ordnung (siehe Abschn. 4.3) in der Empirie beobachtet werden. Für eine höhere Ordnung reichten das Vorhandensein der finanziellen Krise und die Netzwerke der institutionellen Unternehmer*innen nicht aus, da die Hürden und Gewohnheiten bei den Entscheidungsträger*innen zu groß schienen und die Ideen der höheren Ebene weniger kommuniziert wurden. 


\subsection{Vergleich der zwei Fälle}

\subsubsection{Akteur*innen und Ideen}

Die Analyse hat gezeigt, dass in beiden untersuchten transformativen Forschungsprojekten auch tatsächlich eine transformative Intension vorhanden und formuliert war, und dass die Forschenden im Sinne institutioneller Unternehmer*innen agierten und versuchten, ihre Ideen voranzubringen. Zusätzlich dazu verfolgten sie klassische wissenschaftliche Ziele der Erkenntnisgewinnung und nahmen so auch unterschiedliche Rollen gleichzeitig ein.

Bei beiden Fällen zeigt sich, dass die Forschungsinstitutionen über einen guten Ruf in der Stadt verfügten und als epistemische Gemeinschaft (Campbell 2004, S. 104 f.; Haas 1992, S. 27-29) wahrgenommen wurden. Sie verfügten damit über eine gewisse ,power through ideas“ (Carstensen und Schmidt 2016, S. 323326), die ihnen half, ihre Ideen zu verbreiten. Insbesondere die Beteiligung der Institutsleitungen wurde als positiv von den externen Interviewten wahrgenommen und erhöhte die Glaubwürdigkeit der Projekte. Dies ermöglichte auch einen Zugang zu höheren Ebenen in Politik und Unternehmen.

In Bezug auf die Wohlstandsindikatoren wurde die aktive Rolle der Wissenschaftler*innen von den Interviewten positiv gesehen, diese wurden als Berater*innen, Impulsgeber*innen und Netzwerker*innen wahrgenommen, die auch verschiedene Nachhaltigkeitsakteur*innen in der Stadt verstärkt zusammengebracht haben (siehe Tab. 5.1). Als Vorteil von Wissenschaft in diesen und ähnlichen städtischen Prozessen wurde gesehen, dass diese Ideen entwickle, auf die die Verwaltung und Politik selbst nicht kämen und diese Ideen in verschiedene Kontexte einbringen könne. Die Forschenden bei der Wohlstandsindikatorenentwicklung können also auch als Vermittler*innen im Sinne von Campbell (2004, S. 104 f.) zwischen verschiedenen Kontexten - dem wissenschaftlichen und dem der städtischen Verwaltung - gesehen werden.

Auch bei „Glücklich in Wuppertal“ wurden beide Aspekte eingenommen: die der klassischen Forschenden und die der epistemischen Gemeinschaft. Auch hier wurde dies von den Interviewten positiv wahrgenommen und die Wissenschaftler*innen als Berater*innen und Fachleute beschrieben, die außerdem Vernetzung betrieben, das Gespräch suchten und Ideen einbrächten, auf die Stadtverwaltung und -politik selbst nicht komme (siehe Tab. 5.1). 
Tab. 5.1 Rollen der Wissenschaftler*innen

\begin{tabular}{l|l|l}
\hline $\begin{array}{l}\text { Wohlstandsindikatoren für } \\
\text { Wuppertal }\end{array}$ & $\begin{array}{l}\text { App-basiertes Panel } \\
\text { „Glücklich in Wuppertal“ }\end{array}$ & $\begin{array}{l}\text { Zuordnung zu } \\
\text { Akteur*innen nach } \\
\text { Campbell (2004) }\end{array}$ \\
\hline $\begin{array}{l}\text { Forschende } \\
\text { Berater*innen }\end{array}$ & $\begin{array}{l}\text { Forschende } \\
\text { Berater*innen, Fachleute }\end{array}$ & - \\
\hline Impulsgeber*innen & Ideengeber*innen & $\begin{array}{l}\text { Institutionelle } \\
\text { Unternehmer*innen }\end{array}$ \\
\hline Netzwerker*innen & Netzwerker*innen & Vermittler*innen \\
\hline
\end{tabular}

Die Tabelle zeigt die in den Interviews genannten wahrgenommenen Rollen der Wissenschaftler*innen sowie ihre Zuordnung zu den Akteur*innengruppen beim diskursiven Institutionalismus.

Quelle: Campbell (2004) und eigene. Eigene Darstellung.

Die meisten Interviewten meinten, dass die Forschenden dabei auch normativ werden dürften und sahen es auch in diesem Fall als positiv an, dass Wissenschaft dabei innovativere Ideen entwickle, als die Angehörigen aus der Stadtverwaltung selbst. Diese Reflexion über die eigenen Tätigkeiten und das Überschreiten der klassischen Forschendenrolle können als Ansätze dessen verstanden werden, was Schneidewind und Singer-Brodowski (2013, S. 78-81) als Modus-3-Wissenschaft - aufbauend auf der Modus-2-Wissenschaft - bezeichnen (siehe Abschn. 2.2.2): Bei Modus-2-Wissenschaft wird die Trennung zwischen Wissenschaft und Praxis durch Einbeziehung von Praxiswissen teilweise überwunden, um sozial-robustes Wissen zu schaffen, was hier ebenfalls beobachtet werden kann. Bei Modus3-Wissenschaft werden die Forschenden selbst für Veränderungsprozesse aktiv, was in beiden untersuchten Fällen ebenfalls geschah. Ob daraus in diesen Fällen auch eine Veränderung der Wissenschaftseinrichtungen folgte, über die bereits im Vorhinein geschehene Gründung des Transzent hinaus, ist jedoch bisher nicht erkennbar.

In beiden Fällen wurde Wissenschaft also mit ihren Aktivitäten positiv wahrgenommen. Sie scheint sich als institutionelle Unternehmerin zu eignen, die neue Ideen produzieren und in verschiedene Kontexte vermitteln kann. Gleichzeitig wurde auch die Netzwerkfunktion in beiden Fällen als Gewinn angesehen. Bezüglich ihrer Rolle unterschieden sich die Wissenschaftler*innen in den beiden Fällen kaum.

Die Wissenschaftler*innen nahmen also in beiden Fällen einerseits eine klassische Forschendenrolle ein, waren gleichzeitig aber auch aktiv an städtischen Prozessen beteiligt und konnten dabei verschiedenen der im diskursiven 
Institutionalismus beschriebenen Akteur*innengruppen (Campbell 2004) zugeordnet werden. Sie wurden als institutionelle Unternehmer*innen mit alternativen Paradigmen, als Theoretiker*innen und teilweise auch als Vermittler*innen identifiziert. Teilweise versuchten die Wissenschaftler*innen zusätzlich, auch die Rolle von Framer*innen auszufüllen, indem sie die Ideen mit den Wahrnehmungen in der Bevölkerung zusammenbrachten und für die Öffentlichkeit übersetzten. In den untersuchen Fällen haben die Wissenschaftler*innen also versucht, dem Anspruch transformativer Forschung gerecht zu werden, indem sie drei der fünf von Campbell (2004, S. 100-107) unterschiedenen Rollen zugleich ausfüllen und nur teilweise auf die Unterstützung anderer Akteur*innen zurückgriffen. Dies führte dazu, dass die Forschenden sich nicht auf alle Ideen und Ebenen der Kommunikation gleichzeitig fokussieren und nicht allen Rollen gleichzeitig gerecht werden konnten.

Ähnlichkeiten bestehen ebenfalls dahingehend, dass in beiden Fällen die hinter den Projekten stehenden Ideen der institutionellen Unternehmer*innen vor allem auf der Ebene von Paradigmen angesiedelt waren (siehe Abb. 5.3). Konkret zielten die Projekte darauf ab, das vorherrschende Wachstumsparadigma und den Fokus auf wirtschaftliche Entwicklung zu ändern. Statt diese Paradigmen direkt zu kommunizieren, war in beiden Fällen die Herangehensweise, mithilfe der Umsetzung von Ideen erster und zweiter Ebene in Richtung der Änderung des alternativen Paradigmas zu gelangen. Hier zeigt sich die oftmals im Kontext transformativer Forschung beschriebene Annahme, Transformationen würden aus einer Akkumulation verschiedener kleiner Veränderungen entstehen (siehe Abschn. 3.5).

Diese konkreten Anwendungen der unteren Ebenen, beispielsweise bei W2025, waren teilweise nicht zu Beginn der Projekte geplant, sondern haben sich erst im Laufe der Projektentwicklung ergeben, weil Anschlussmöglichkeiten an städtische Prozesse sichtbar wurden. Kleine konkrete Umsetzungen konnten hier ermöglicht werden, was leichter erschien, als eine Diffusion des alternativen Paradigmas in städtischen Diskursen. In beiden Fällen zeigte sich, dass die Wissenschaftler*innen über städtische Prozesse Bescheid wussten und so konkrete Möglichkeiten ergreifen konnten, wenn Projekte und institutionelle Veränderungen greifbar waren. Gleichzeitig waren ihre Ideen insoweit diffundiert, dass intermediäre Akteur*innen darüber Bescheid wussten und die Forschenden in laufende Prozesse hereinholten. Bei diesen Anwendungen, beispielsweise beim Bürgerbudget, wurden dann von Seiten der Wissenschaft konkrete Vorschläge für die Umsetzung gemacht, was laut Campbell (2004, S. 118, siehe Abschn. 3.3.2) 


\section{Stabilität Gradueller Wandel Transformativer Wandel}

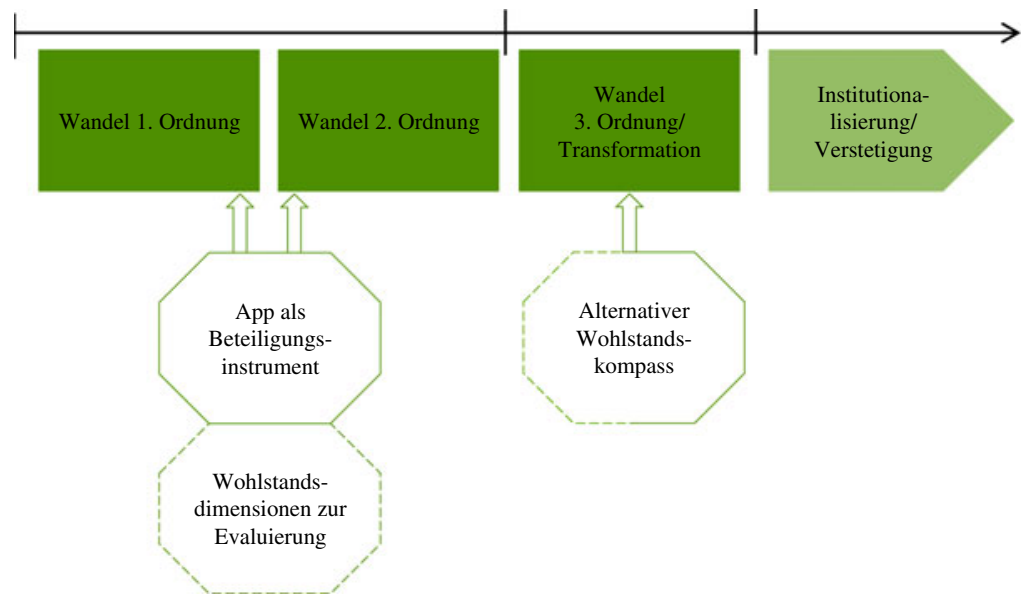

Abb. 5.3 Zuordnung der Ideen zu Veränderungsintensionen im Vergleich. Die Abbildung zeigt die Zuordnung der Ideen der institutionellen Unternehmer*innen in beiden Fällen im Vergleich (durchgängige Linie: „Glücklich in Wuppertal“, gestrichelte Linie: Wohlstandsindikatoren) zu drei Graden der Veränderung. Die Wohlstandsdimensionen oder auch dazugehörige Indikatoren zur Evaluierung von Stadtentwicklungsprojekten oder die App als Beteiligungsinstrument zu nutzen, würde je nach Ausgestaltung einen Wandel erster oder zweiter Ordnung bedeuten, während die Einführung eines alternativen Wohlstandskompasses eine Transformation mit sich bringen würde.

Quelle: Hall (1993) und eigene. Eigene Darstellung.

eine Voraussetzung für das Aufgreifen neuer Programme durch Entscheidungsträger*innen ist. Dies geschah größtenteils, ohne die alternativen Paradigmen dahinter explizit zu kommunizieren.

Hier erscheint es so, als ob die Forschenden ein an die MLP angelehntes Transformationsverständnis vertreten und so versucht haben, aus der Nische heraus kleine Innovationen anzustoßen und damit das Regime zu verändern (Geels 2002, 2011; Kemp und van Lente 2013, siehe Abschn. 2.2.1). In der Theorie des diskursiven Institutionalismus (siehe Abschn. 3.3.2) bleibt unklar, ob Veränderungen auf den ersten beiden Ebenen auf dem Weg zu Veränderungen dritter Ordnung helfen. An einigen Stellen wird in der Theorie aber darauf hingedeutet, dass es eher nicht zu einem Paradigmenwechsel kommt, wenn Veränderungen erster oder zweiter Ordnung vorgenommen wurden und die wahrgenommene Krise 
damit bereits zufriedenstellend gelöst wurde (Hall 1993, S. 280). Eine Implementierung von Policies und Programmen könnte also sogar kontraproduktiv sein, wenn das Ziel eine Veränderung von Paradigmen ist. Eine öffentliche Debatte über neue Paradigmen könnte dagegen Veränderung befördern (Hall 1993, S. 286; Hay 2001, S. 200). Laut Campbell (2004, S. 104 f.) bringen Vermittler*innen neue Ideen wie Paradigmen und Politikprogramme von der Forschung direkt zur Politik, verbreiten sie aber auch in der Presse, um öffentliche Diskussionen zu beeinflussen. Dafür hätte es bei den untersuchten Fällen allerdings eine Thematisierung der alternativen Paradigmen in öffentlichen Diskursen gebraucht, was jedoch in beiden Fällen kaum geschehen ist.

Dies trifft insbesondere auf „Glücklich in Wuppertal“ zu, wo zwar Ideen auf den unterschiedlichen Ebenen vorhanden waren, jedoch wurden die Ideen der ersten und zweiten Ebenen vordergründig kommuniziert und die App unter anderem als neues Instrument dargestellt. Der Gestaltungsanspruch dahinter auch auf der Ebene von Paradigmen wurde seltener kommuniziert und deshalb auch nur von sehr wenigen Personen wahrgenommen. Bei dem ersten untersuchten Fall, der Entwicklung von Wohlstandsindikatoren, war dies zwar etwas ausgeglichener, durch die insgesamt weniger ausgeprägte Kommunikationstätigkeit kamen jedoch auch hier nur bei wenigen Akteur*innen die alternativen Paradigmen an.

Zielgruppe der meisten Dokumente war die Bürgerschaft in Wuppertal. Da wie eben erwähnt in demokratischen Gesellschaften der Kampf um konkurrierende Paradigmen unter anderem in der Öffentlichkeit stattfindet, erscheint eine Ansprache der Bürgerschaft und eine breite Presseberichterstattung in beiden Fällen sinnvoll, wenn ein Paradigmenwechsel angestrebt wird. Bei der App war dies zusätzlich deswegen wichtig, weil Teilnehmende gewonnen werden mussten. Jedoch ist in den öffentlichen Dokumenten an die Bürgerschaft kaum klar formulierte Kritik an bisherigen Ideen und vorherrschenden Paradigmen enthalten, sondern die Projekte werden eher als zusätzliche Angebote und Instrumente dargestellt. Eine große Zahl an Dokumenten, wenngleich weniger als an die Bürgerschaft, richten sich an Wissenschaftler*innen oder Wissenschaftsförderer, wodurch die klassische Rolle der Forschenden, die üblichen Adressaten ihrer Publikationen und ihre Aufgaben in der Bearbeitung von Drittmittelprojekten deutlich werden.

Erstaunlich ist, dass bei beiden Fällen kein Dokument sich explizit an die Stadtverwaltung oder Stadtpolitik richtet. Die Kommunikation fand eher informell am Rande von Besprechungen und mündlich statt. Bei den Indikatoren wäre mehr Kommunikation an die Entscheidungsträger*innen zu erwarten gewesen, denn eine Implementierung der Wohlstandsindikatoren in der Stadt setzt auf Veränderungen innerhalb der Verwaltung und Politik. Auch „Glücklich in Wuppertal“ 
hatte zum Ziel, die Ergebnisse der App für die Stadt nutzbar zu machen. Dieser Kontakt zu Entscheidungsträger*innen ist jedoch in beiden Fällen eher oberflächlich geblieben - einerseits, weil die Projektlaufzeit kurz war und andererseits, weil von Seiten der Entscheidungsträger*innen kein großes Interesse wahrgenommen wurde.

Am besten funktionierte die Zusammenarbeit mit den Entscheidungsträger*innen dort, wo es um konkrete Projekte ging, bei denen die Stadtverwaltung selbst einen Vorteil in der Kooperation sah, beispielsweise beim STEK2030Prozess in Kooperation mit "Glücklich in Wuppertal“ und beim Bürgerbudget mit der Nutzung der Wohlstandsindikatoren. Hier war die Stadt auf der Suche nach konkreten Kriterien beziehungsweise einem Beteiligungsinstrument, so dass es leichter fiel, die Projekte zu integrieren. Als förderlich stellte sich außerdem heraus, wenn die institutionellen Unternehmer*innen aus der Wissenschaft die Umsetzung selbst durchführten, anstatt nur Vorschläge zu formulieren. Bei beiden Fällen ging der Kontakt zur Zivilgesellschaft und zu den Entscheidungsträger*innen meist von den Forschenden aus, in seltenen Fällen wurden diese aus der Stadtverwaltung heraus angesprochen. Erfolgreicher waren die Verbreitung und Umsetzung der Ideen, wenn die Wissenschaft eine aktive Rolle einnahm.

In beiden analysierten Fällen wurden an die Entscheidungsträger*innen Angebote formuliert und nicht konkrete Forderungen; zudem ist kaum Kritik an den vorherrschenden Paradigmen bei den Entscheidungsträger*innen angekommen. Ein Interviewter formulierte sogar selbst, die Wissenschaft könnte, wenn sie ihre Instrumente der Stadtverwaltung zur Verfügung stellt - wie die App - auch Forderungen stellen, wie damit verfahren werden sollte (siehe Abschn. 5.2.1.6).

„Glücklich in Wuppertal“ konnte bei der Kommunikation auf ein breiteres Netzwerk mit einer größeren Reichweite zurückgreifen als die Wohlstandsindikatorenentwicklung, insbesondere durch die angesehenen und lokal gut vernetzten Unternehmenspartner*innen, die die Verankerung in der Stadt erleichterten (siehe Tab. 5.2). Hier agierten beide stadtnahen Unternehmenspartner*innen als Framer*innen, was eine Verbreitung der Ideen auf den unteren beiden Ebenen und eine Verknüpfung mit den öffentlichen Empfindungen vereinfachte. Durch die Gastbeiträge in der WZ wurde "Glücklich in Wuppertal" in den Zusammenhang mit persönlichen Geschichten von Zeitungsredakteur*innen und lokalen Persönlichkeiten über ihr Glück gerückt. Allerdings wurde nur ein Ausschnitt der Ideen vermittelt und das alternative Paradigma nicht kommuniziert. Indem die Sparkasse das Design der App entwickelte und Werbung auf ihren Kanälen machte, agierte sie ebenfalls als Framerin. Bei „Glücklich in Wuppertal“ gab es daher im Vergleich wesentlich mehr Kommunikation in der Öffentlichkeit und somit eine breitere Diffusion der Ideen, jedoch oftmals nur mit wenigen Informationen. So 


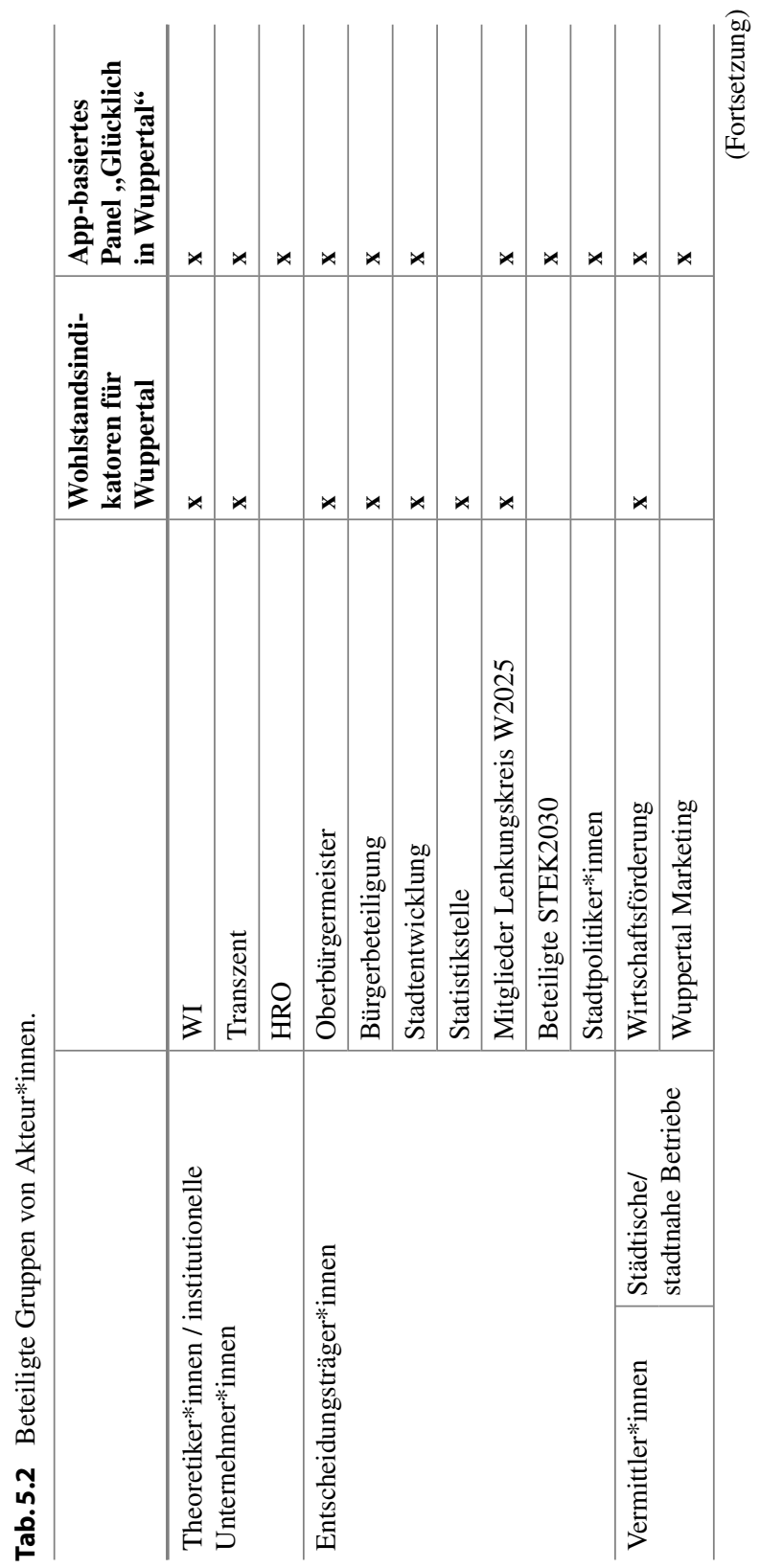




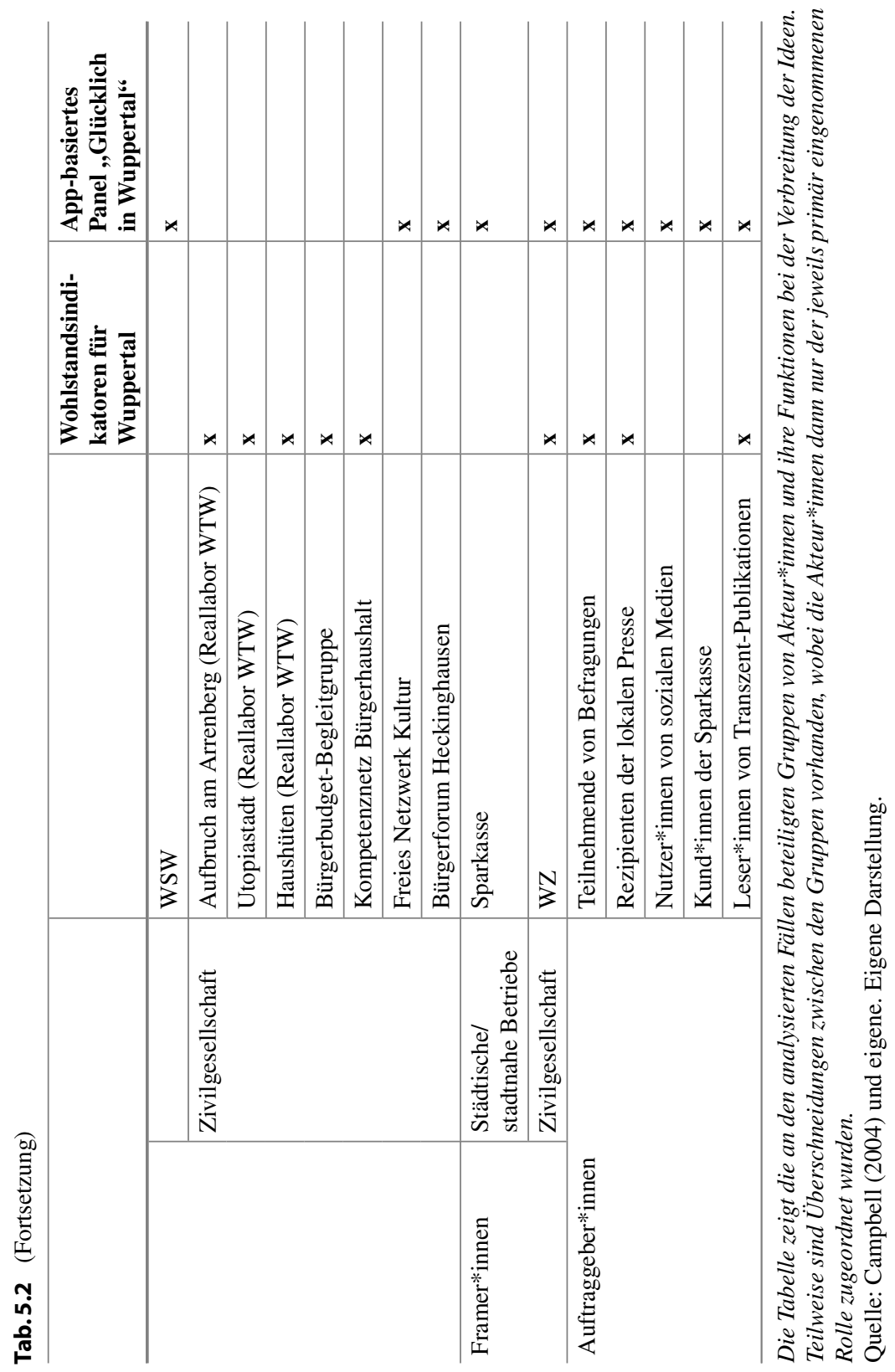


wurde viel über die Möglichkeit der Teilnahme an den Befragungsrunden und wenig über das alternative Paradigma informiert.

Die App stellte sich als anschlussfähiger an die Ideen der Entscheidungsträger*innen heraus, wenn es um konkrete Themen wie Abfragen zu verkehrspolitischen Planungen oder um ein Bürgerbeteiligungsinstrument ging, als wenn es sich um allgemeinere Trends in der Zufriedenheit handelte. Außerdem schien es schwierig zu sein, bei stadtpolitischen Prozessen tiefgehend einzusteigen, da beispielsweise die Treffen der W2025-Gruppe sehr kurz für die Menge an besprochenen Projekten waren und so nur einen Teil der Ideen zu „Glücklich in Wuppertal“" an die Entscheidungsträger*innen herangetragen wurde. Als Vermittler*innen und Unterstützer*innen haben sich bei „Glücklich in Wuppertal“ insbesondere die WZ sowie zwei Vertreter*innen von städtischen Betrieben herausgestellt, die die Ideen der App in andere Kontexte getragen haben. Weitere intermediäre Akteur*innen aus Zivilgesellschaft und Unternehmen wurden nur vereinzelt angesprochen.

Bei der Indikatorenentwicklung ist insgesamt weniger Diffusion der Ideen in der Öffentlichkeit und zu Entscheidungsträger*innen zu beobachten. Diese wurden hauptsächlich über die eigenen Kanäle und Netzwerke verbreitet, die im Fall der Indikatorenentwicklung wesentlich kleiner waren als bei „Glücklich in Wuppertal" und hauptsächlich den Bereich der Zivilgesellschaft umfassten. So konnte die Vernetzung zur Zivilgesellschaft Erfolge verzeichnen, auch wenn die Projektinhalte für viele noch unkonkret und unklar blieben. Darüber hinaus wussten auch einige Entscheidungsträger*innen darüber Bescheid und entschieden sich in einzelnen Fällen sogar für die Nutzung. Als Vermittler*innen und Unterstützer*innen haben sich zwei zivilgesellschaftliche Akteur*innen und eine Vertreterin eines städtischen Betriebes herausgestellt. Kontakt zu Unternehmen bestand darüber hinaus in diesem Fall nicht, so dass auch hier kaum weitere intermediäre Akteur*innen mobilisiert wurden, die als Vermittler*innen oder Framer*innen agieren konnten.

Bezüglich der Kommunikation in die Öffentlichkeit hinein wurden die Begriffe Wohlstand und gutes Leben als weniger klar wahrgenommen, weshalb sich die Kommunikation dieser Ideen und Projektziele als schwieriger herausstellte (siehe Abschn. 5.1.1.5). Der Begriff Wohlstand wurde weniger oft von anderen Akteur*innen aufgegriffen als die Formulierung gutes Leben. Der Begriff Glück dagegen ist besser angekommen, wurde als einfach verständlich wahrgenommen und in vielen Presseberichten verwendet. Doch es gab auch Kritik daran, Glück sei ein zu kurzfristiges und subjektives Gefühl, an dem sich Politik und Verwaltung nicht unbedingt orientieren sollten (siehe Abschn. 5.2.3). Alle drei Begriffe 
stellten sich als diffus heraus und wurden mit unterschiedlichen Bedeutungen versehen.

Deutlich wird außerdem ein Unterschied in der Darstellung der Forschungsergebnisse zwischen den Wohlstandsindikatoren und „Glücklich in Wuppertal“: Bei den Indikatoren wurde erst sehr spät die Auswahl der Dimensionen und Indikatoren kommuniziert, die konkrete Berechnung blieb bis zum Projektende in den veröffentlichten Broschüren und Webseiten unvollständig. Einige Interviewte hätten sich konkrete Berichte gewünscht. Diese späte Ergebnisdarstellung war zu einem Teil im Projektantrag angelegt, weil der Fokus auf dem partizipativen Entwicklungsprozess der Indikatoren lag. Später verzögerte sie sich dann weiter, was auf die Fülle der parallelen Aufgaben der Wissenschaftler*innen zurückzuführen ist. Bei „Glücklich in Wuppertal“" waren erste Ergebnisse direkt nach Ende der ersten Erhebungswelle online in einem Dashboard abrufbar, jedoch hätten sich auch in diesem Fall einige Entscheidungsträger*innen eine konkrete Beschreibung der Ergebnisse in einer für Stadtverwaltung und -politik einfacher nutzbaren, knappen Form gewünscht. Bei „Glücklich in Wuppertal“ stellte es sich außerdem als schwierig heraus, Nutzer*innen langfristig zu gewinnen, weshalb die bisherigen Daten bislang nicht die gewünschte Qualität und Repräsentativität erreicht haben.

In beiden Fällen waren die Ergebnisse also nicht früh genug oder nicht in zufriedenstellender Form und Qualität verfügbar. Für die Implementierung und Bekanntmachung der Projekte wäre wesentlich mehr Zeit nötig gewesen. Die Förderlaufzeiten der Projekte wurden als zu kurz angesehen, um nach einer fundierten wissenschaftlichen Analyse noch genügend Zeit zur Verfügung zu haben, um wirklich Wandel anstoßen zu können und die Ideen und die entwickelten Instrumente in der Stadt zu verankern. Sollte dies gleichzeitig geschehen, schien der transformative dem wissenschaftlichen Beitrag teilweise entgegenzustehen, für zwei nacheinander laufende Prozesse schien die Laufzeit zu kurz zu sein (siehe u. a. Abschn. 5.1.1.5).

Zusammenfassend lässt sich sagen, dass beide Fälle zum Ziel hatten, Ideen unterschiedlicher Ebenen, von Policies über Programme bis hin zu Paradigmen, zu verbreiten (siehe Tab. 5.3). Insbesondere war es ein Ziel, ein neues Konzept ressourcenleichten Wohlstandes dem Wachstumsgedanken und dem Fokus auf ökonomische Aspekte in städtischen Entwicklungen entgegenzustellen. In beiden Fällen wurde dazu jedoch kaum ein kritischer öffentlicher Diskurs angestoßen, sondern eher Ideen auf der ersten und zweiten Ebene kommuniziert und teilweise umgesetzt. "Glücklich in Wuppertal" ist dabei weiter in städtischen Diskursen diffundiert als die Wohlstandsindikatoren. In beiden Fällen wurden die Forschenden als epistemische Gemeinschaft positiv wahrgenommen und konnten an einige städtische Prozesse anknüpfen. Dies gelang bei „Glücklich in Wuppertal“ leichter, 
Tab. 5.3 Vergleich der beiden Fälle in Bezug auf Ideen und Akteur*innen.

\begin{tabular}{|c|c|c|}
\hline & $\begin{array}{l}\text { Wohlstandsindikatoren } \\
\text { für Wuppertal }\end{array}$ & $\begin{array}{l}\text { App-basiertes Panel } \\
\text { „Glücklich in Wuppertal“" }\end{array}$ \\
\hline $\begin{array}{l}\text { Ebenen der Ideen } \\
\text { (Veränderungsintension) }\end{array}$ & $\begin{array}{l}\text { Insb. Paradigmen, auch } \\
\text { Policies, Programme }\end{array}$ & $\begin{array}{l}\text { Insb. Paradigmen, auch } \\
\text { Policies, Programme }\end{array}$ \\
\hline $\begin{array}{l}\text { Ebenen der diffundierten } \\
\text { Ideen }\end{array}$ & Insbesondere Policies & $\begin{array}{l}\text { Insbesondere Policies und } \\
\text { Programme }\end{array}$ \\
\hline $\begin{array}{l}\text { Institutionelle } \\
\text { Unternehmer*innen }\end{array}$ & Wissenschaftler*innen & $\begin{array}{l}\text { Wissenschaftler*innen, } \\
\text { teilweise Unternehmen }\end{array}$ \\
\hline Ressourcen & Vorhanden & Vorhanden \\
\hline $\begin{array}{l}\text { Zugang zu / Unterstützung } \\
\text { durch } \\
\text { Entscheidungsträger*innen }\end{array}$ & $\begin{array}{l}\text { Zu einzelnen, wenig } \\
\text { Unterstützung }\end{array}$ & $\begin{array}{l}\text { Zu einzelnen, wenig } \\
\text { Unterstützung }\end{array}$ \\
\hline Netzwerke & $\begin{array}{l}\text { Eng, insb. zu organisierter } \\
\text { Zivilgesellschaft, teilweise } \\
\text { Stadtverwaltung }\end{array}$ & $\begin{array}{l}\text { Breit, insb. in Bürgerschaft } \\
\text { hinein, Medien, teilweise } \\
\text { Stadtverwaltung }\end{array}$ \\
\hline Vermittler*innen & $\begin{array}{l}\text { Einzelne aus stadtnahem } \\
\text { Betrieb und } \\
\text { Zivilgesellschaft }\end{array}$ & $\begin{array}{l}\text { Einzelne aus stadtnahen } \\
\text { Betrieben und Medien }\end{array}$ \\
\hline Framer*innen & Keine & $\begin{array}{l}\text { Stadtnahe Unternehmen, } \\
\text { Medien }\end{array}$ \\
\hline Projektspezifische Faktoren & $\begin{array}{l}\text { Verwendete Begriffe schwer } \\
\text { greifbar; } \\
\text { Ergebnisse sehr spät } \\
\text { veröffentlicht }\end{array}$ & $\begin{array}{l}\text { Verwendete Begriffe gut } \\
\text { greifbar; } \\
\text { Ergebnisse schon früh } \\
\text { veröffentlicht; } \\
\text { Schwierigkeit, Nutzer*innen } \\
\text { für App zu gewinnen und zu } \\
\text { halten }\end{array}$ \\
\hline
\end{tabular}

Die Tabelle zeigt im Vergleich der beiden Fälle, auf welchen Ebenen Ideen vorhanden waren und wie weit und zu wem sie diffundierten. Daneben fasst sie zusammen, mit wem die institutionellen Unternehmer*innen vernetzt waren und von wem sie Unterstützung erhielten, sowie einige projektspezifische Faktoren.

Quelle: Campbell (2004) und eigene. Eigene Darstellung.

da auf breitere Netzwerke zurückgegriffen werden konnte und der Glücksbegriff anschlussfähiger war. In den untersuchten Fällen wurden die Ideen dort erfolgreich verbreitet und umgesetzt, wo Vermittler*innen oder Framer*innen aus Zivilgesellschaft oder stadtnahen Betrieben zwischen den Wissenschaftler*innen und den Entscheidungsträger*innen standen und die Ideen in die entsprechenden 
Kontexte übersetzten, so dass die Wissenschaftler*innen selbst diese Rolle nicht zusätzlich einnehmen mussten.

Überraschend ist, dass kein Dokument der Forschenden direkt an Entscheidungsträger*innen gerichtet ist, sondern meist die Bürgerschaft adressiert wurde und dass bei beiden Fällen die Kommunikation von den Forschungsergebnissen und Ideen als nicht ausreichend wahrgenommen wurde. Insbesondere trifft dies auf die Wohlstandsindikatoren zu, wo die Kommunikation der eigentlichen Indikatoren erst sehr spät und nur unvollständig geschah. An welche städtischen Prozesse und Krisen die beiden Fälle mit ihren Ideen anknüpfen konnten und bei welchem der beiden Fälle dies besser gelang, wird im folgenden Abschnitt dargestellt.

\subsubsection{Rahmenbedingungen und Anknüpfungspunkte}

$\mathrm{Zu}$ den Rahmenbedingungen und Entwicklungen in der Stadt Wuppertal zählten in beiden Fällen Krisen (Campbell 2004, S. 115; Hay 2006, S. 67), die neue Lösungen erfordern und öffentlich diskutiert werden. Größtenteils wurden dieselben wahrgenommenen Krisen im Zusammenhang mit beiden untersuchten Fällen erwähnt. Manche Prozesse oder Krisen wurden jedoch auch nur im Zusammenhang mit einem der Fälle genannt, da nur hier von den Interviewten mögliche Anknüpfungspunkte gesehen wurden (siehe Tab. 5.4).

In beiden Fällen wurden insbesondere die Verschuldung Wuppertals und der dadurch oft geringe Entscheidungsspielraum von Stadtpolitik und -verwaltung hervorgehoben. Außerdem nahmen die Interviewten eine hohe Armutsquote, das schlechte Image der Stadt und auch ein negatives Selbstbild der Stadt wahr. Diese Herausforderungen wurden als Krisen wahrgenommen, stellten jedoch keine kurzfristigen Probleme dar, sondern langfristige Rahmenbedingungen der Stadt. Hier konnten beide Fälle anschließen, indem sie andere Faktoren von Lebensqualität als wirtschaftliche Zahlen in den Vordergrund rückten und insbesondere auch die positiven Aspekte der Stadt hervorhoben. $\mathrm{Zu}$ einer konkreten Nutzung der Indikatoren, mit denen die Stadtverwaltung ihre Entwicklungen beschreibt oder auf Grundlage derer Entscheidungen getroffen werden, kam es jedoch nicht.

Die grundlegendere Frage, wie Lebensqualität in einer vom Strukturwandel betroffenen Stadt unter der Notwendigkeit einer Nachhaltigkeitstransformation aussehen könnte, wurde nur von den institutionellen Unternehmer*innen, also den Forschenden, formuliert, schaffte aber keine Verknüpfung mit den genannten Krisen. Woran dies liegt und was dies für die Thesen des diskursiven Institutionalismus bedeutet, wird in Abschnitt 6.1 genauer diskutiert. Daneben wurde eine 


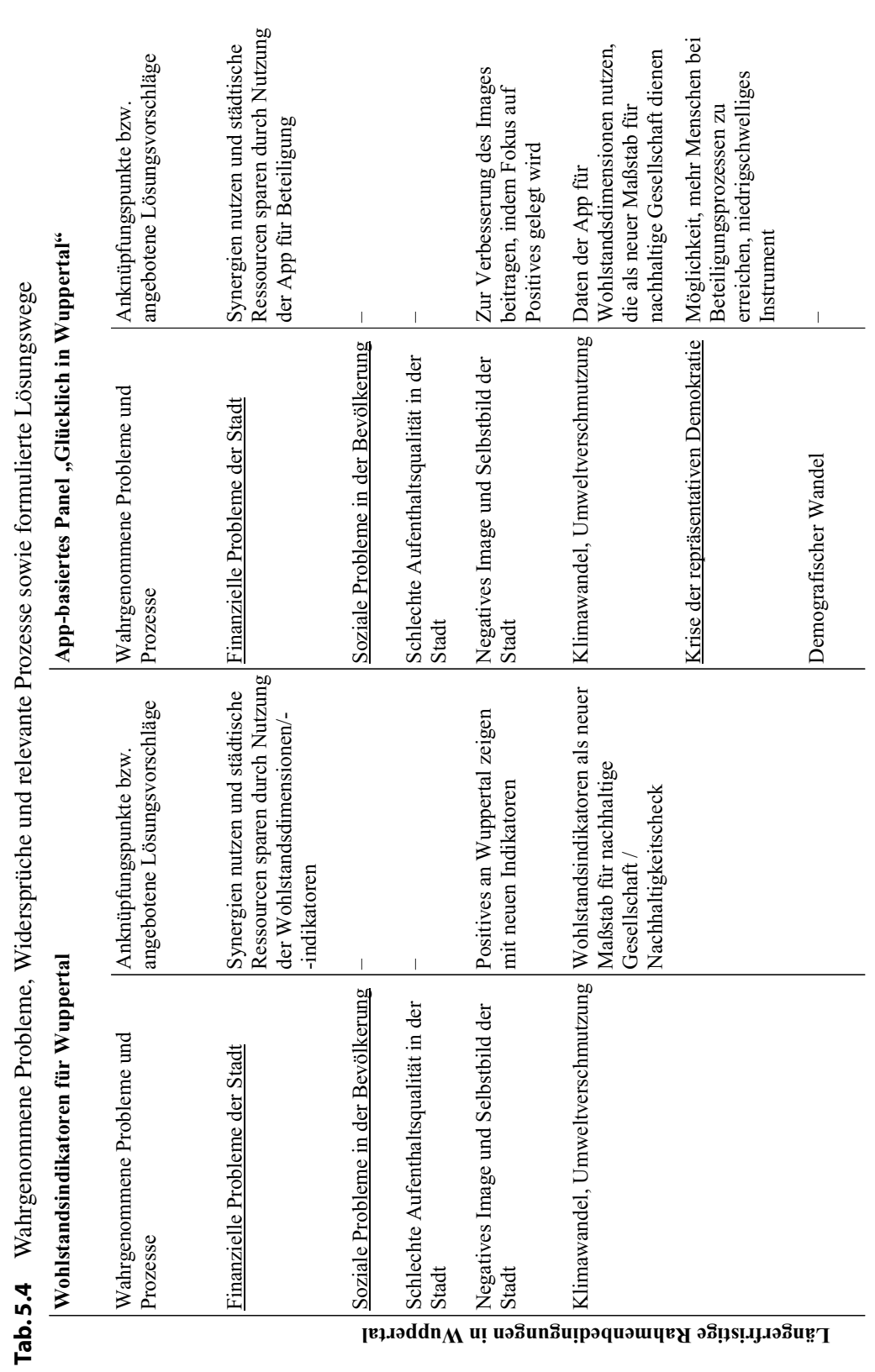



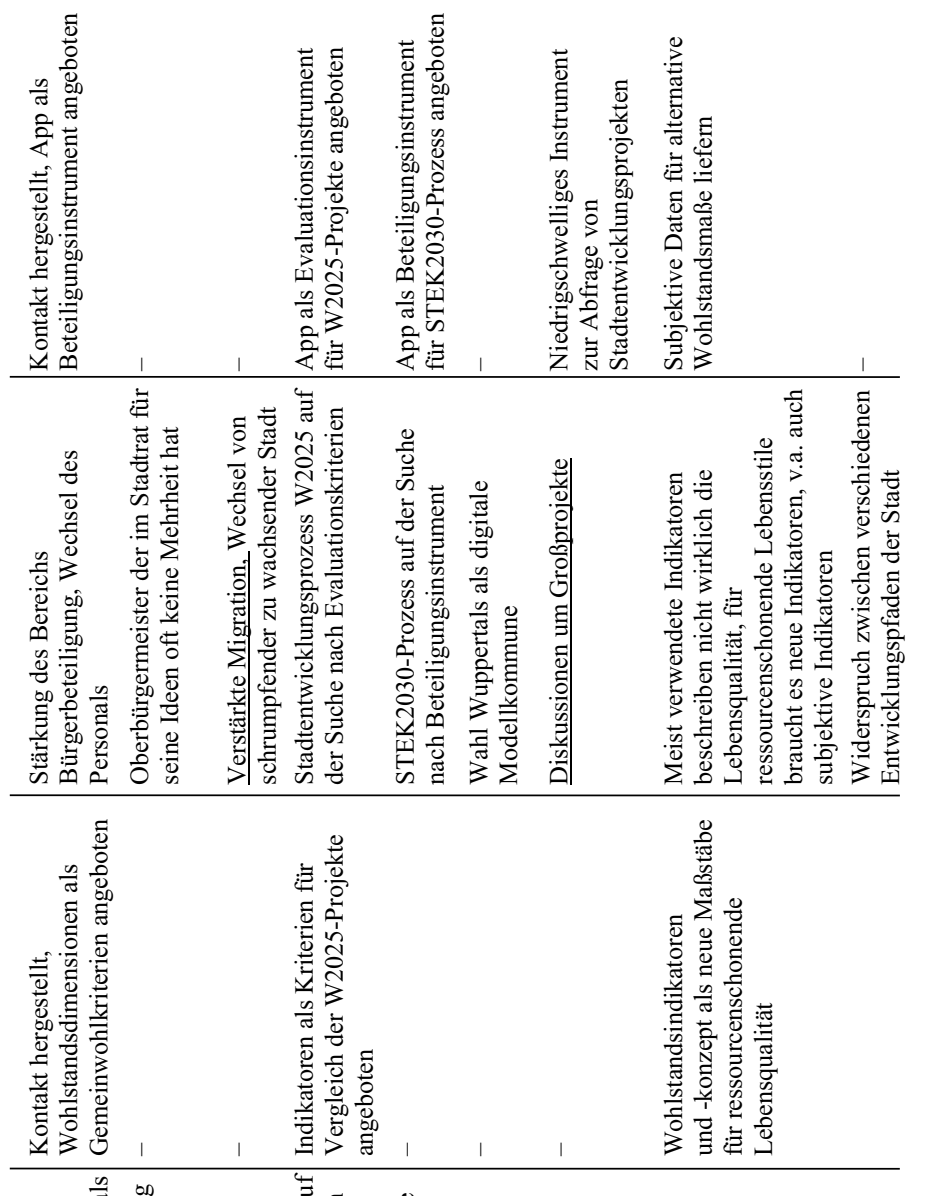

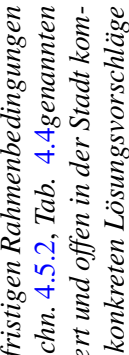

$2 \pi \frac{1}{2} 5$

: $.5 \frac{2}{2}$

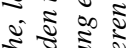

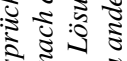

욜 ₹

ฐ)

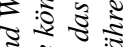

₹ $\frac{7}{2}: 3$

2. $\frac{2}{5} \frac{2}{2}$

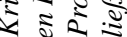

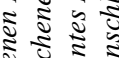

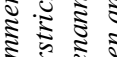

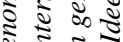

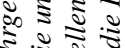

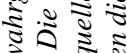

)

$\frac{3}{2} \frac{2}{2} \frac{2}{2}$

ธิ㇒

ป

ำ

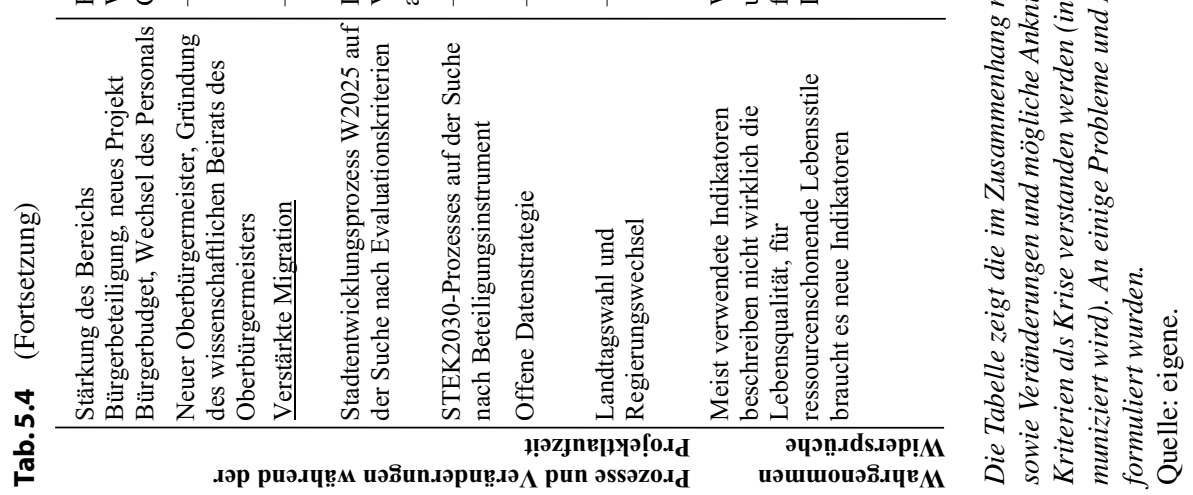


Krise im Kontext von Bürgerbeteiligung und Vertrauen in die demokratischen Institutionen wahrgenommen: Es werde immer schwerer, die Bürger*innen zu erreichen und für Bürgerbeteiligung zu motivieren, außerdem sinke das Vertrauen in städtische Institutionen. Hieran wurde in Bezug auf „Glücklich in Wuppertal“ eine Anschlussmöglichkeit gesehen, da die App gegebenenfalls ein niedrigschwelliges Beteiligungsinstrument darstellen könnte, das diese Menschen erreichen könnte. Doch auch dieses Instrument müsste sich erst bewähren und zeigen, dass es für die Stadt vorteilhaft genutzt werden kann, Ressourcen einspart und trotzdem ein repräsentatives Bild erzeugt. Hier erfolgte die Anknüpfung also auf den niedrigsten Ebenen von Ideen als Policy oder Programm.

Des Weiteren konnten die beiden Fälle teilweise an institutionelle Veränderungen in der Stadt anknüpfen, die sich im Laufe der Projektlaufzeit entwickelten. So stellte die Neugründung des Bürgerbeteiligungsdezernats zunächst eine gute Anschlussmöglichkeit dar, wenngleich dieses kurz darauf aufgelöst, in eine Stabsstelle umgewandelt wurde und es mehrere Wechsel der Mitarbeitenden gab. Diese neu geschaffene Institution, die eine Schnittstelle zwischen Stadtverwaltung und Bürgerschaft darstellt, könnte im Sinne einer durchlässigen Institution verstanden werden, die es institutionellen Unternehmer*innen eher erlaubt, sich einzubringen (Campbell 2004, S. 178). Die Stärkung des Bereichs Bürgerbeteiligung durch die neuen Mitarbeitenden ermöglichte eine Zusammenarbeit zwischen dem Projekt der Wohlstandsindikatoren und einem der ersten Beteiligungsprojekte der Stabsstelle: einem Bürgerbudgetprozess. Weitere städtische Prozesse waren W2025 und STEK2030, was insbesondere für die App Anknüpfungspunkte lieferte, auch hier auf der Ebene von Policies und Programmen. Daneben wurden weitere Prozesse wie die digitale Modellkommune und die Wahl eines neuen Oberbürgermeisters genannt, bei denen ein Anschließen bisher jedoch nicht erfolgreich war.

Besonders anschlussfähig sind beide Projekte außerdem an die im Themenbereich Nachhaltigkeit engagierte Zivilgesellschaft in der Stadt; weniger anschlussfähig an die Mehrheit der Gesellschaft, was insbesondere bei den Wohlstandsindikatoren angemerkt wurde. Die Akteur*innen im Nachhaltigkeitsbereich sahen die Wohlstandsindikatoren insbesondere als geeignete Grundlage für einen Nachhaltigkeitscheck, der seit einiger Zeit ein Ziel dieser Akteur*innen ist. Hier wäre eine Anknüpfung auf der höchsten Ebene von Ideen angesiedelt, was jedoch auch die großen Hürden gegenüber einer Umsetzung erklärt. Im Fokus beider Fälle standen der Kontakt zu Entscheidungsträger*innen und weniger eine vertiefte Entwicklung der Ideen in Nischen. In diesen Nischen von Nachhaltigkeitsakteur*innen wurden die Ideen höherer Ebene positiv aufgenommen und Anknüpfungsmöglichkeiten gesehen, da sie passend für deren Paradigmen und Empfindungen waren. Bei den Entscheidungsträger*innen war dagegen noch ein 
Fokus auf ökonomische Ziele verankert, weshalb die neuen Paradigmen dort weniger gut anknüpfen konnten.

Die Analyse beider Fälle deutet darauf hin, dass die genannten Krisen von den Entscheidungsträger*innen bisher nicht als so gefährlich für die Macht- und Ressourcenverteilung angesehen und die vorgeschlagenen Paradigmen nicht als bessere Lösungen und zur Vergrößerung ihrer Macht und Ressourcen angesehen wurden, als dass sie einen Wandel aktiv unterstützen würden. Laut Theorie des diskursiven Institutionalismus kann es besonders in Krisenzeiten dazu kommen, dass ein vorherrschendes Paradigma die Entwicklungen nicht mehr erklären kann und die Durchsetzung eines alternativen Paradigmas gelingt (Hall 1993, S. 285). Dass dies nicht erfolgte, liegt möglicherweise daran, dass es sich nicht um eine neu hinzugekommene Krise handelt, sondern ein längerfristiges Problem. Bisher boten sich immer neue Policies und Programme an, so dass die Ebene der Paradigmen noch nicht angetastet werden musste.

\subsubsection{Beobachtbare Veränderungen}

Nach drei Jahren Projektlaufzeit konnte beobachtet werden, dass einige Akteur*innen in der Stadt die Begriffe gutes Leben und Wohlstand vermehrt nutzten. Im ersten untersuchten Fall - der Entwicklung von Wohlstandsindikatoren kam es zu kleinen diskursiven Veränderungen. Auch die oft negative Selbstbeschreibung der Stadt konnte zu einem geringen Teil ins positive gedreht werden. Beim Bürgerbudget kann bereits eine Umsetzung eines Teils der Ideen verzeichnet werden, wobei es sich jedoch nicht um ein längerfristiges Instrument handelt, sondern lediglich um eine einmalige Verwendung der Dimensionen. Bei W2025 wurde sich für die Nutzung entschieden. Da der Prozess dann aber insgesamt ins Stocken geraten ist, kam es letztlich nicht zu einer Verwendung der Indikatoren.

Im W2025-Prozess entschied man sich zusätzlich für eine Nutzung von „Glücklich in Wuppertal“, die jedoch auch in diesem Fall letztendlich nicht realisiert wurde. Doch auch die App wurde einmal in einem städtischen Prozess genutzt, in diesem Fall beim STEK2030-Prozess. Im Laufe der drei Jahre hat auch dieser zweite Fall einen Diskurs angestoßen. Durch die häufige Berichterstattung in der WZ ist dieser sogar noch sehr viel weiter in die Öffentlichkeit gedrungen. Die Diffusion geschah allerdings nur bei einem kleinen Teil der Ideen von „Glücklich in Wuppertal“ und es war kein kritischer Diskurs auf der Ebene neuer Paradigmen, sondern eher auf das Thema Glück allgemein und auf die App als Instrument bezogen. 
Beide Fälle transformativer Forschung haben kleine Veränderungen in den Diskursen sowie vereinzelte neue Instrumente in der Stadtpolitik, also Veränderungen erster Ordnung, bewirkt. Inwieweit diese zu einer Veränderung dritter Ordnung einer Transformation - beitragen können, ist jedoch unklar. Bisher ist es dazu in beiden Fällen nicht gekommen. Die Ergebnisse deuten darauf hin, dass die Ideen alternativer Paradigmen nicht weit genug verbreitet wurden, um mit den kleinen Umsetzungen den Weg hin zu einer Transformation zu bereiten.

Open Access Dieses Kapitel wird unter der Creative Commons Namensnennung 4.0 International Lizenz (http://creativecommons.org/licenses/by/4.0/deed.de) veröffentlicht, welche die Nutzung, Vervielfältigung, Bearbeitung, Verbreitung und Wiedergabe in jeglichem Medium und Format erlaubt, sofern Sie den/die ursprünglichen Autor(en) und die Quelle ordnungsgemäß nennen, einen Link zur Creative Commons Lizenz beifügen und angeben, ob Änderungen vorgenommen wurden.

Die in diesem Kapitel enthaltenen Bilder und sonstiges Drittmaterial unterliegen ebenfalls der genannten Creative Commons Lizenz, sofern sich aus der Abbildungslegende nichts anderes ergibt. Sofern das betreffende Material nicht unter der genannten Creative Commons Lizenz steht und die betreffende Handlung nicht nach gesetzlichen Vorschriften erlaubt ist, ist für die oben aufgeführten Weiterverwendungen des Materials die Einwilligung des jeweiligen Rechteinhabers einzuholen.

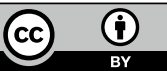

